\title{
Analytical study for double-layer geosynthetic reinforced load transfer platform on
} column improved soft soil

\section{(1)}

(1)

(1)

6

7.
${ }^{3}$ Associate Professor of Geotechnical Engineering, School of Civil and Environmental Engineering, University of Technology Sydney (UTS), Sydney, Australia, Email: hadi.khabbaz@uts.edu.au \footnotetext{
Hong Kong Polytechnic University, Hung Hom, Kowloon, Hong Kong, China, Email: jian-

${ }^{4}$ Chair Professor of Soil Mechanics, The Department of Civil and Environmental, The hua.yin@polyu.edu.hk
}

*Corresponding Author, School of Civil and Environmental Engineering Faculty of Engineering and Information Technology University of Technology Sydney (UTS)

City Campus PO Box 123 Broadway NSW 2007

$$
\mathrm{T}(+61)(2) 95147883 \mathrm{~F}(+61)(2) 95142633 \mathrm{M} 0413573481
$$

Email: behzad.fatahi@uts.edu.au 


\title{
Analytical study for double-layer geosynthetic reinforced load transfer platform on
} column improved soft soil

\author{
Balaka Ghosh $^{1}$, Behzad Fatahi ${ }^{2 *}$, Hadi Khabbaz ${ }^{3}$, and Jian-Hua Yin ${ }^{4}$
}

\section{ABSTRACT}

The objective of this study is to propose a reasonably accurate mechanical model for double-layer geosynthetic reinforced load transfer platform (LTP) on column reinforced soft soil which can be used by practicing engineers. The developed model is very useful to study the behaviour of LTP resting on soft soil improved with conventional columns such as concrete columns, piles, and deep soil mixing columns. The negligible tensile strength of granular material in LTP, bending and shear deformations of LTP, compressibility and shearing of soft soil have been incorporated in the model. Furthermore, the results from the proposed model simulating the soft soil as Kerr foundation model are compared to the corresponding solutions when the soft soil is idealised by Winkler and Pasternak foundation models. It is observed from the comparison that the presented model can be used as a tool for a better prediction of the LTP behaviour with multi layers of geosynthetics, in comparison with the situation that soft soil is modelled by Winkler and Pasternak foundations. Furthermore, parametric studies show that as the column spacing increases, the maximum deflection of LTP and normalised tension in the geosynthetics also increase. Whereas, the maximum deflection of LTP and normalised tension in the geosynthetics decrease with increasing LTP thickness, stiffness of subsoil, and stiffness of geosynthetic reinforcement. In addition, it is observed that the use of one stronger geosynthetic layer (e.g. $1 \times 2000 \mathrm{kN} / \mathrm{m}$ ) with the equivalent stiffness of two geosynthetic layers (e.g. $2 \times 1000 \mathrm{kN} / \mathrm{m}$ ) does not result in the same settlement of LTP and the tension of the geosynthetic reinforcement when compared to two weaker geosynthetic layers.

Keywords: Geosynthetics; Soil-structure interaction; Timoshenko beam; Load transfer platform; Multilayer; Soft soil 


\section{Introduction}

Insufficient bearing capacity and excessive settlement are very common and severe issues of soft soils when heavy superstructures are constructed on the top of these soils (Parsa-Pajouh et al., 2016). Thus, in combination with cautious field observations and laboratory tests, the use of ground improvement techniques using rigid (e.g. concrete injected columns, jet grouted columns, and piles) or semi-rigid inclusions (e.g. deep soil mixing columns and lime-cement columns) has grown substantially over the last two decades (Bergado et al., 1999; Han et al., 2004). Load transfer platform (LTP), a layer of sand or gravel consisting of geosynthetic layers, is commonly placed over the columns (e.g. concrete injected columns, or piles) used for ground improvement to facilitate the load transfer from the superstructures to the columns (Russell and Pierpoint, 1997; Han and Gabr, 2002; Kempfert et al., 2004).

Application of a load transfer platform resting on column improved soft soil is very common, particularly when highway embankments are built on improved ground. To analyse the column supported embankments, several analytical models have been proposed in the literature. Van Eekelen et al. (2013) summarised and classified them as (a) frictional models (Terzaghi, 1943; McKelvey, 1994; Russell and Pierpoint, 1997; Naughton, 2007; McGuire et al., 2012), (b) rigid arch models (Carlsson, 1987; Rogbeck et al., 1998; Svanø et al.; 2000; Van Eekelen et al., 2003), (c) models using mechanical elements (Deb, 2010; Filz et al.; 2012; Zhang et al., 2012a, b; Deb and Mohapatra, 2013) and (d) limit-state equilibrium models (Marston and Anderson, 1913; Hewlett and Randolph, 1988; Jones et al., 1990; Zaeske, 2001). British design guidelines BS8006 (2010), discussed by Van Eekelen et al. (2011), adopted the empirical model proposed by Jones et al. (1990) to study the geosynthetic reinforced column supported embankments. Zaeske's model (2001) latter was adopted in the German design 
73 guidelines EBGEO (2010). Van Eekelen et al. (2013) proposed a new limit-state 74 equilibrium model for piled embankments which is an extension of the model proposed by Hewlett and Randolph (1988) and EBGEO (2010). Several other researchers compared the results of existing analytical models with field or laboratory measurements (Chen et al., 2008; Chen et al., 2010; Briançon and Simon, 2012; Girout et al., 2016). Chen et al. (2008) conducted experiments both with and without geosynthetics and compared the results of their experiments with existing analytical models, namely Terzaghi (1943) and Low et al. (1994) and the original 2D equation of Marston and Anderson (1913). Zaeske (2001), Heitz (2006), and Farag (2008) compared the results of their laboratory model tests with their predictions from the calculations. Results of a predictive model to capture membrane behaviour of the geosynthetic reinforcement based on the results of twelve model tests have been reported by Van Eekelen et al. (2012a, b). Several other studies have been conducted using two dimensional numerical models of geosynthetic reinforced column supported embankment structures adopting the finite element method (FEM) and finite difference method (FDM) (Han et al., 2007; Huang et al., 2009; Huang and Han, 2010; Yapage and Liyanapathirana, 2014). Furthermore, the predictions adopting full-width model were compared with unit cell model in numerical simulations by Bhasi and Rajagopal (2015), Khabbazian et al. (2015), and Yu and Bathurst (2017). Collin et al. (2005) proposed a mechanical model of multiple layers of low strength geogrids within the LTP based on the concept of "beam" theory. But, the interrelationship between the embankment settlement and strain in the geosynthetics was ignored in that study. However, application of a load transfer platform is not limited to the column supported embankments. Load transfer platform is widely used for heavy superstructures such as fuel tanks and silos. The practical designs of LTP demand the simple yet accurate 
modelling of (i) the mechanical behaviour of the LTP, (ii) the mechanical behaviour of the underneath soft soil, and (iii) the interaction mechanism between the LTP and the soft soil.

$$
\text { While physically close and mathematically simple idealisations of the mechanical }
$$
behaviour of the geosynthetic reinforced granular fill or LTP can be established adopting Timoshenko (Yin, 2000a, b; Shukla and Yin, 2003; Zhao et al., 2016) or the Euler-Bernoulli beam theories (Maheshwari et al., 2004; Maheshwari and Viladkar, 2009; Zhang et al., 2012a, b) or even the Pasternak shear layer theory (Yin, 1997a, b; Deb et al., 2007; Deb, 2010), the characteristics that represent the mechanical behaviour of the soft soil and its interaction with the granular layer are difficult to model. Since in reality, the soft soil is heterogeneous, anisotropic and nonlinear in load-displacement response, the simple springs cannot simulate the soil response accurately. It should be noted that the most commonly used mechanical model to simulate the soil is the one developed by Winkler (1867). Although, the model proposed by Van Eekelen et al. (2013) can be applicable for both full and partial arching which results in a better representation of the arching measured in the experiments than the other existing models such as EBGEO (2010), BS8006 (2010), especially when the embankment is relatively thin, Van Eekelen et al. (2013) modelled the subsoil as an elastic spring with constant modulus of subgrade reaction which is comparable to linear Winkler's springs. Winkler's idealisation symbolises the soil medium as a series of identical but mutually independent, closely spaced, linearly elastic spring elements. Since according to the Winkler hypothesis, there is no interaction between adjacent springs, this model cannot account for the dispersion of the load with depth and distance from the loading area. However, it is a common phenomenon that the surface deflections occur not only immediately under the loaded region but also within certain limited regions beyond the 
123 loaded area. Therefore, Winkler's model has the inability to take into account the 124 continuity or shear strength of the soil. Hence, compressibility of the soil was 125 considered in the model proposed by Van Eekelen et al. (2013) while shear action in 126 the soil was ignored. To overcome the weaknesses of the Winkler's model (i.e. to 127 achieve some degree of interaction between the individual spring elements), some 128 modified foundation models have been suggested in the literature. In these modified 129 models, a second parameter was introduced to Winkler foundation to eliminate the 130 discontinuous behaviour of soil by providing continuity through interaction between 131 the individual spring elements with some structural elements (Filonenko-Borodich, 132 1940; Hetényi, 1946; Pasternak, 1954). To further improve the two-parameter 133 foundation models, the third soil parameter was introduced, leading to the so-called 134 "three-parameter" foundation model. Among several three-parameter foundation models, the foundation model proposed by Kerr (1965) is of particular interest since it 136 geneses from the well-known Pasternak foundation model for which several 137 applications and solutions have been already available in the literature. Kerr foundation 138 model consists of two spring layers, with varied spring constants, interconnected by a 139 shear layer. Furthermore, Kerr concluded that for different types of foundation 140 materials (e.g. soil and foam), the Winkler foundation model cannot realistically predict 141 the interaction mechanisms between the beams and the contacting soil medium. 142 Therefore, the most important task for practicing engineers is to simulate soft soil, 143 which demands simple modelling but provides an accurate response of the soft soil.

144 Mechanical behaviour of the geosynthetic reinforced granular fill or LTP can be 145 theoretically established by adopting the Pasternak shear layer theory (Yin, 1997a, b; 146 Deb et al., 2007; Deb, 2010), the Euler-Bernoulli beam theory (Maheshwari et al. 2004; 147 Maheshwari and Viladkar, 2009; Zhang et al., 2012a, b), and the Timoshenko beam 
149 theory, the cross-section of the LTP does not rotate and therefore, the granular layer experiences transverse shear deformation only. Thus, bending deformation of the

151 granular layer was ignored in the developed models (Yin, 1997a, b; Deb et al., 2007;

152 Deb, 2010). For application of the Euler-Bernoulli theory in geosynthetic reinforced 153 soil (Maheshwari et al. 2004; Maheshwari and Viladkar, 2009; Zhang et al., 2012a, b), 154 by considering the plane sections remain plane and perpendicular to the neutral axis after deformation, the shear deformation of a geosynthetic reinforced soil was ignored. However, after deformation of beams with the small length - to depth ratio, the cross section of the beam is still not be perpendicular to the neutral axis. To overcome the shortcomings of Euler-Bernoulli and Pasternak theories, the well-known Timoshenko (1921) beam can be adopted to simulate the LTP (Yin, 2000a, b). Yin (2000a, b) idealised the soft soil, the granular layer, and the geosynthetics by linear Winkler

161 springs, Timoshenko beam, and a rough membrane, respectively. Based on the 162 Timoshenko (1921) beam assumption, Yin's model considers the shear and the flexural

163 deformations of the granular layer since the rotation between the cross section and the 164 bending line of the beam is acceptable. However, the model considered a linear 165 behaviour for soft soil, and the infinite tensile stiffness for the granular fill materials 166 was assumed while column supports were not considered. Zhao et al. (2016) proposed 167 a new dual beam model for a geosynthetic-reinforced granular fill with an upper 168 pavement. Zhao et al. (2016) modelled the upper pavement by an Euler-Bernoulli beam, 169 while the geosynthetic reinforced granular fill was simulated by a reinforced 170 Timoshenko beam. The explicit derivation process for the behaviour of this dual beam-

171 foundation system was presented in this study and an exact solution was suggested.

172 However, effects of columns and negligible tensile strength of soil were not considered 
173 in that study. When the granular material in LTP is dense to very dense (relative density

174 greater $\geq 65 \%$ ) due to the compaction process, idealisation of LTP as Timoshenko

175 beam is more appropriate (Shukla and Yin, 2003). Indeed, the total settlement of LTP

176 can occur due to the beam bending mechanism as well as the shear action, similar to

177 the case of a reinforced concrete beam. After a few years of operation, LTP will become

178 stiffer and behave like a concrete beam, deforming in shear as well as in bending.

179 Hence, the settlement analysis of LTP in the construction stage or short time after may

180 be conducted using the existing models (Deb, 2010; Van Eekelen et al. 2013), but the

181 model proposed in this paper can be more suitable for the latter stages of LTP life as

182 well as construction stage or short time after construction (by assuming lower shear or

183 bending stiffness of LTP).

184 Most of the analytical and numerical studies related to geosynthetic reinforced

185 granular layer on soft soil have been conducted for the single layer geosynthetic

186 reinforced soil system (Yin, 1997a, b; Maheshwari et al., 2004; Huang and Han, 2009;

187 Zhao et al., 2016), while very limited number of studies have addressed multilayer 188 geosynthetic reinforced arrangement (Nogami and Yong, 2003; Liu and Rowe, 2015;

189 Van Eekelen et al., 2015; Borges and Gonçalves, 2016). Nogami and Yong (2003)

190 proposed a mechanical model for a multilayer geosynthetic reinforced soil subjected to

191 structural loading. Nogami and Yong (2003) considered each soil layer by a system of

192 an infinite number of closely spaced one-dimensional columns connected with

193 horizontal springs. Governing differential equations were solved iteratively by the finite

194 difference method. Therefore, the present study is an attempt to suggest a generalised

195 model that provides a closed-form solution to estimate the behaviour of multilayer

196 reinforced granular fill. 

behaviour of LTP on column reinforced soft soil by idealising the physical modelling of the LTP on the soil media as "membrane reinforced Timoshenko beam" on Kerr

200 foundation. The analytical model developed in this study can be applied by practicing 201 engineers to predict the deflection of the LTP and mobilised tension in the geosynthetic 202 reinforcement. Then, an analytical solution for the governing differential equation is 203 proposed. The suitability of the Kerr foundation model for engineering calculations of 204 LTP are evaluated while LTP is subjected to symmetric loading. To solve the governing 205 differential equations, the supports of column in the reinforced soft soil is counted in 206 by considering the reaction force in the column locations. To validate the proposed 207 model, the results from the proposed model simulating the soft soil as the Kerr 208 foundation model are compared to the corresponding solutions when the soft soil is 209 idealised by Winkler and Pasternak foundations. Similar approach to validate the 210 analytical model was taken by several other researchers available in the literature 211 (Maheshwari and Viladkar, 2009; Zhang et al., 2012b; Lei et al., 2016). Parametric 212 studies are also carried out to assess the overall behaviour of the multilayer geosynthetic 213 reinforced granular layer as well as that of the single layer geosynthetic reinforced 214 granular layer.

\section{2. Formulation of the problem}

216 The proposed mechanical model that idealises the mechanistic behaviour of a load 217 transfer platform (LTP) on column improved soft soil in plane strain condition is 218 presented in Fig. 1a. The free body diagrams of the small segments in LTP (i.e. element 219 A) and shear layer (i.e. element B) of length $d x$ are shown in Figs. 1b-c, respectively. 220 In this study, double layers of geosynthetic reinforcement embedded within compacted 221 granular layers are considered. The geosynthetic reinforcement is modelled as a rough 
elastic membrane, placed inside the Timoshenko beam representing the granular fill materials. Thus, the combined representation of the geosynthetic-reinforced granular layer is a structural element named as "membrane-reinforced Timoshenko beam". Columns and soft soil are idealised by Winkler springs and Kerr foundation model, respectively. It is implicit here that granular fill material in the load transfer platform (LTP) has insignificant tensile strength compared to compressive strength, so similar to a concrete beam, tension cracks are expected to spread from the tension face (bottom edge of LTP) in the direction of the neutral axis in the span. In contrast, since the granular layer is continuous over the column positions, the direction of the bending 231 moment changes adjacent to the columns. Accordingly, tension cracks are produced at 232 the top edge of the granular layer and spread towards the neutral axis. A typical profile 233 of deflection of the LTP assumed for the analytical development is shown in Fig. 2a. 234 After cracking, it may be presumed that plane sections continue to be plane, but as the 235 load increases, these cracks spread towards the neutral axis, and then the neutral axis 236 starts to change its position depending on tension cracks propagation. It is assumed here 237 that the flexural cracks are developed vertically. Since some parts of the granular layer 238 are cracked, the soil in those fractured zones cannot sustain tensile stresses and becomes 239 weaker. Therefore, geosynthetic reinforcement is embedded to strengthen the granular 240 fill. Similar approach (i.e. cracked load transfer platform) was considered previously 241 by Ghosh et al. (2016) while load transfer platform was analysed on Winkler foundation 242 considering the non-linear behaviour of soft soils. For the sake of obtaining an 243 analytical solution and following one of the basic assumptions used for flexural design 244 of reinforced concrete beams, it is presumed that the geosynthetic reinforcement is 245 attached to the granular material, thus it is reasonable to assume that the tensile and 246 compressive forces mobilised in LTP are carried by geosynthetic reinforcement and 
granular material, respectively. This means the strain in the geosynthetic reinforcement

248 is equal to the strain in the granular fill at the same level. It should be noted that by 249 making this simplifying assumption, possible gap or slip between the geosynthetics and 250 the granular fill materials is ignored. A similar assumption was adopted by several other 251 researchers to study the mechanical behavior of LTP (Yin 2000a, b; Shukla and Yin, 252 2003). Hence, section properties of a cracked LTP should be adopted for flexural 253 design. Since the initiation of the tension cracks and their propagation are varied in 254 different locations, the design of LTP would be more accurate if different cross section properties in different locations of LTP are considered, depending on the locations of 256 the tension cracks. Considering the position of the tension cracks, the loaded LTP is 257 divided into two sections, as shown in Fig. 2a. Region I (when $-r \leq x \leq+r$ ) where tension cracks in the LTP appear from the bottom edge; which means the bottom of LTP is under tension (sagging moment). In contrast, in Region II (when $\pm r \leq x \leq$ $260 \pm s / 2$ ), tension cracks in the LTP develop from the top edge (hogging moment). Figs. $2612 b-c$ illustrate the effective cross sections of the LTP in Regions I and II, respectively. 262 The cracked transformed section to carry out the flexural analysis is attained by 263 substituting the area of geosynthetic reinforcement with an equivalent area of granular 264 fill material equal to $n A_{r}$, where $n\left(n=E_{r} / E_{g}\right)$ is the modular ratio with the elastic 265 modulus of geosynthetic reinforcement $\left(E_{r}\right)$ and granular fill material $\left(E_{g}\right)$ and $A_{r}$ is 266 the cross sectional area of geosynthetic reinforcement. To analyse the response of LTP, 267 the neutral axis is located first, positioned at a distance $\left(h_{s}\right)$ from the compression end 268 of LTP in the sagging bending moment region which is indicated in Fig. 2b. The first 269 moment of the compression area in the LTP $\left(A_{s}\right)$ above the neutral axis with respect to 270 neutral axis must be equal that of the tension area in the transformed geosynthetic 271 layer $\left(n A_{r}^{b}\right)$ under the neutral axis; that is $A_{s} h_{s} / 2=n A_{r}^{b}\left(y_{r}^{b}+y_{s}\right)$. where $A_{r}^{b}$ is the 
cross-section area of bottom geosynthetic reinforcement; $y_{r}^{b}$ is the locations of bottom

273 geosynthetic layer from the centroid axis; and $y_{s}$ is the distance between neutral axis 274 and centroid axis of LTP within the sagging bending moment section. The above275 mentioned equation is a quadratic equation in terms of $h_{s}$, the value of which 276 determines the location of the neutral axis. Similarly, to establish the neutral axis $\left(h_{h}\right)$ 277 in the hogging region, first moment of the compression area in the LTP $\left(A_{h}\right)$ above the 278 neutral axis with respect to neutral axis must be equal that of the tension area in the 279 transformed geosynthetic layer $\left(n A_{r}^{t}\right)$ below the neutral axis. To acquire the depth of 280 the neutral axis $\left(h_{s}\right.$ or $\left.h_{h}\right)$, the solutions of the resulting quadratic equations are found 281 as follows:

$$
h= \begin{cases}h_{s}=\sqrt{\left(\frac{s_{r}^{b}}{E_{g}}\right)^{2}+\left[\frac{s_{r}^{b}}{E_{g}}\left(2 y_{r}^{b}+h\right)\right]}-\left(\frac{s_{r}^{b}}{E_{g}}\right), & -r \leq x \leq+r \\ h_{h}=\sqrt{\left(\frac{s_{r}^{t}}{E_{g}}\right)^{2}+\left[\frac{s_{r}^{t}}{E_{g}}\left(2 y_{r}^{t}+h\right)\right]}-\left(\frac{s_{r}^{t}}{E_{g}}\right), & \pm r \leq x \leq \pm \frac{s}{2}\end{cases}
$$

282 where $h$ is the thickness of LTP before cracking; $h_{s}$ and $h_{h}$ are the locations of neutral 283 axis in sagging moment and hogging moment zones, respectively; $y_{r}^{t}$ and $y_{r}^{b}$ are the 284 locations of top and bottom geosynthetic layer from the centroid axis, respectively; $285 S_{r}^{t}\left(=A_{r}^{t} E_{r}^{t}\right)$ and $S_{r}^{b}\left(=A_{r}^{b} E_{r}^{b}\right)$ are the tensile stiffness of top and bottom geosynthetic 286 layers, respectively; $E_{r}^{t}$ and $E_{r}^{b}$ are the Young's moduli of top and bottom 287 reinforcements, respectively; $E_{g}$ is the Young's modulus of the granular material; and $288 A_{r}^{t}$ and $A_{r}^{b}$ are the cross-sectional area of top and bottom geosynthetic reinforcements, 289 respectively

290 After locating the neutral axis, the equivalent bending stiffness of the granular layer 291 with geosynthetic reinforcement $\left(D_{s}\right.$ and $\left.D_{h}\right)$ is calculated as follows. 


$$
D= \begin{cases}D_{s}=E_{g} I_{s}+S_{r}^{b}\left(y_{s}+y_{r}^{b}\right)^{2}, & -r \leq x \leq+r \\ D_{h}=E_{g} I_{h}+S_{r}^{t}\left(y_{h}+y_{r}^{t}\right)^{2}, & \pm r \leq x \leq \pm \frac{s}{2}\end{cases}
$$

Although in flexure, the existence of granular materials below/above the neutral axis is omitted, but the same granular material between the neutral axis and the cracks is needed for shear transfer between the geosynthetic reinforcement and the compression zone. Hence, the shear stiffness of the granular fill including geosynthetic 296 reinforcement $(C)$ can be calculated as follows.

$$
C=k_{s c}\left\{\frac{E_{g} h}{2\left(1+v_{g}\right)}+\frac{S_{r}^{t}}{2\left(1+v_{r}^{t}\right)}+\frac{S_{r}^{b}}{2\left(1+v_{r}^{b}\right)}\right\}, \quad-\frac{s}{2} \leq x \leq+\frac{s}{2}
$$

297 where $y_{s}$ and $y_{h}$ are the distances between neutral axis and centroid axis of LTP within 298 the sagging and hogging bending moment sections, respectively; $v_{\mathrm{g}}, v_{r}^{t}$, and $v_{r}^{b}$ are 299 the Poisson's ratios of granular material, top and bottom geosynthetic layers, 300 respectively; $D_{s}$ and $D_{h}$ are the equivalent bending stiffness of LTP within the sagging 301 and hogging bending moment sections, respectively; $C$ is the shear stiffness of LTP 302 irrespective of the sagging and hogging bending moments; $I_{s}$ and $I_{h}$ are the second 303 moment of inertias of the granular materials within the sagging and hogging bending 304 moment sections, respectively $\left(I_{s}=h_{s}{ }^{3} / 3\right.$ and $\left.I_{h}=h_{h}{ }^{3} / 3\right)$; and $k_{s c}$ is the shear factor 305 suggested by Cowper (1966) and Hutchinson (2001) for the rectangular cross section 306 of a beam.

307 As the LTP settles on the column improved soft soil, shear stresses are generated 308 in the soft soil. Thus, Winkler foundation model to simulate the soft soil under the LTP 309 would not be suitable in this case as the differential settlement occurs underneath the 310 granular layer. Because of the discontinuity amongst the spring elements, Winkler 311 foundation model cannot consider the shear stress transfer in the soil. Hence, for the 312 sake of realistic modelling of the soft soil, the connectivity of the individual Winkler 
springs must be achieved through a structural element such as a beam, a shear layer, or

314 a plate. However, this structural element cannot be introduced just below the granular

315 layer. Since the differential settlement of soft soil just underneath the granular layer is

316 very high, large shear stresses are generated in this region. However, since soil is a

317 continuum medium, the differential settlement dissipates over the soil depth, resulting

318 in less shear stresses generated in the soft soil. Therefore, structural elements such as a

319 shear layer must be introduced in combination with the Winkler springs at some

320 distance below the granular layer. Hence, the Kerr foundation model which consists of

321 two spring layers interconnected by a shear layer is adopted to simulate the soft soil.

322 The three-parameter Kerr foundation model consists of two linear spring layers with

323 modulus of subgrade reactions $k_{u}$ and $k_{l}$, interconnected by a shear layer with shear

324 modulus $G$ (as shown in Fig. 1a). Plane strain condition allowing the consideration of

325 a LTP strips of finite length " $s$ " and unit width, is considered. To analyse the LTP, the

326 equilibrium equations (i.e. externally applied loads equal to the sum of the internal

327 element forces at all nodes of a structure) and the compatibility equations (i.e. one or

328 more equations which state either that no gaps exist internally or deflections are

329 consistent with the geometry imposed by the supports) which are the most fundamental

330 equations in structural analysis. Therefore, the concept of "Load-Displacement

331 compatibility method" in the present research is adopted from fundamental laws of

332 physics. Similar concept was implemented by Smith (2005) and Filz and Smith (2007)

333 for design of bridging layers in geosynthetics reinforced embankments. Hence, to

334 satisfy the vertical deformation continuity, the following conditions should be satisfied.

$$
w_{L T P}= \begin{cases}w_{s}^{L T P}=w_{s}^{u s}+w_{s}^{l s}, & -r \leq x \leq+r \\ w_{h}^{L T P}=w_{h}^{u s}+w_{h}^{l s}, & \pm r \leq x \leq \pm \frac{s}{2}\end{cases}
$$


where $w_{s}^{L T P}$ and $w_{h}^{L T P}$ are the deflections of the LTP in the sagging and hogging

336 regions, respectively; $w_{s}^{u s}$ and $w_{s}^{l s}$ are the contractions or extensions of the upper and

337 lower springs layers in the sagging region, respectively; $w_{h}^{u s}$ and $w_{h}^{l s}$ are the contraction

338 or extension of the upper and lower spring layers in the hogging region, respectively.

339 The contact pressures $(q)$ under the LTP as shown in Fig. $1 \mathrm{~b}$ can be expressed as:

$$
q= \begin{cases}q_{s}=k_{u} w_{s}^{u s}, & -r \leq x \leq+r \\ q_{h}=k_{u} w_{h}^{u s}, & \pm r \leq x \leq \pm \frac{s}{2}\end{cases}
$$

The governing equation for the Pasternak shear layer as displayed in Fig. 1c is

341 given by:

$$
q= \begin{cases}q_{s}=k_{l} w_{s}^{l s}-G w_{s}^{l s^{\prime \prime}}, & -r \leq x \leq+r \\ q_{h}=k_{l} w_{h}^{l s}-G w_{h}^{l s^{\prime \prime}}, & \pm r \leq x \leq \pm \frac{s}{2}\end{cases}
$$

342 where $k_{u}$ and $k_{l}$ are the spring constants for upper and lower layers, respectively and

$343 G$ is the shear modulus of soft soil. According to Lagrange's notation, a prime mark

344 denotes a derivative (e.g. $\left.w_{s}^{l s^{\prime \prime}}=\frac{d^{2} w_{s}^{l s}}{d x^{2}}\right)$.

345 Rearranging Eqs. (5a) and (5b), the relationship between the deflection of the upper 346 soil layer and the contact pressure at the interface of LTP and soft soil can be obtained 347 as below:

$$
\frac{k_{l}}{k_{u}} q_{s}-\frac{G}{k_{u}} q_{s}^{\prime \prime}=k_{l} w_{s}^{u s}-G w_{s}^{u s^{\prime \prime},}, \quad-r \leq x \leq+r
$$

348 and

$$
\frac{k_{l}}{k_{u}} q_{h}-\frac{G}{k_{u}} q_{h}^{\prime \prime}=k_{l} w_{h}^{u s}-G w_{h}^{u s^{\prime \prime}}, \quad \pm r \leq x \leq \pm \frac{s}{2}
$$

Combining Eqs. (6a) and (7a) and then substituting the resulting equation in Eq.

350 (4a), leads the relationship between the deflection of the LTP and the contact pressure 351 at the interface of LTP and soft soil in sagging region which is stated in Eq. (8a) (similar 352 steps are applied for Eq. (8b)): 


$$
\left(1+\frac{k_{l}}{k_{u}}\right) q_{s}-\frac{G}{k_{u}} q_{s}^{\prime \prime}=k_{l} w_{s}^{L T P}-G w_{s}^{L T P^{\prime \prime}}, \quad-r \leq x \leq+r
$$

353 and

$$
\left(1+\frac{k_{l}}{k_{u}}\right) q_{h}-\frac{G}{k_{u}} q_{h}^{\prime \prime}=k_{l} w_{h}^{L T P}-G w_{h}^{L T P^{\prime \prime}}, \quad \pm r \leq x \leq \pm \frac{s}{2}
$$

The differential equations for a LTP in the plane strain condition adopting membrane reinforced Timoshenko (1921) beam can be rewritten as:

$$
D_{s} w_{s}^{L T P^{i v}}-\frac{D_{s}}{C} q_{s}^{\prime \prime}+q_{s}=p-\frac{D_{s}}{C} p^{\prime \prime}, \quad-r \leq x \leq+r
$$

356 and

$$
D_{h} w_{h}^{L T P^{i v}}-\frac{D_{h}}{C} q_{h}^{\prime \prime}+q_{h}=p-\frac{D_{h}}{C} p^{\prime \prime}, \quad \quad \pm r \leq x \leq \pm \frac{s}{2}
$$

Combining Eqs. (8a) and (9a) yields the governing differential equation of the

359 below.

$$
\begin{aligned}
& \left(\frac{G D_{s}}{k_{u}}\right) w_{s}^{L T P^{v i}}-D_{s}\left(1+\frac{k_{l}}{k_{u}}+\frac{G}{C}\right) w_{s}^{L T P^{i v}}+\left(\frac{D_{s} k_{l}}{C}+G\right) w_{s}^{L T P^{\prime \prime}}-k_{l} w_{s}^{L T P}= \\
& -\left(\frac{G D_{s}}{C k_{u}}\right) p^{i v}+\left(\frac{D_{s}}{C}+\frac{D_{s} k_{l}}{C k_{u}}+\frac{G}{k_{u}}\right) p^{\prime \prime}-\left(1+\frac{k_{l}}{k_{u}}\right) p
\end{aligned}
$$

360 where Roman numerals, as in $w_{S}^{L T P^{v i}}, w_{S}^{L T P^{i v}}$, and $w_{S}^{L T P^{\prime \prime}}$ denote sixth, fourth, and 361 second order derivatives with respect to $x$, respectively.

362 Similarly, combining Eqs. (8b) and (9b), the response of LTP in the hogging region

363 (i.e. for $\pm r \leq x \leq \pm s / 2$ ) can be represented as:

$$
\begin{aligned}
& \left(\frac{G D_{h}}{k_{u}}\right) w_{h}^{L T P^{v i}}-D_{h}\left(1+\frac{k_{l}}{k_{u}}+\frac{G}{C}\right) w_{h}^{L T P^{i v}}+\left(\frac{D_{h} k_{l}}{C}+G\right) w_{h}^{L T P^{\prime \prime}}-k_{l} w_{h}^{L T P}= \\
& -\left(\frac{G D_{h}}{C k_{u}}\right) p^{i v}+\left(\frac{D_{h}}{C}+\frac{D_{h} k_{l}}{C k_{u}}+\frac{G}{k_{u}}\right) p^{\prime \prime}-\left(1+\frac{k_{l}}{k_{u}}\right) p
\end{aligned}
$$

\section{The analytical solutions}

In the present study, two-dimensional plane strain analysis has been carried out for

366 column-supported structures. Analytical solutions are obtained for calculating the 
settlement of the load transfer platform at any arbitrary point for the symmetric loading condition. Fourier series is utilised to consider the symmetric distribution of vertical loading $(p)$ on LTP between the two adjacent columns. Hence, $p$ can be described as:

$$
p=P_{0}+\sum_{n=1}^{n=\infty} P_{n} \cos \left(\frac{2 n \pi x}{s}\right)
$$

370 where

$$
P_{0}=\frac{1}{s} \int_{-s / 2}^{s / 2} f(x) d x \text { and } P_{n}=\frac{2}{s} \int_{-s / 2}^{s / 2} f(x) \cos \left(\frac{2 n \pi x}{s}\right)
$$

Region I (i.e. for $-r \leq x \leq+r$ ).

$$
\begin{aligned}
& w_{s}^{L T P^{v i}}+X_{s} w_{s}^{L T P^{i v}}+Y_{s} w_{s}^{L T P^{\prime \prime}}+Z_{s} w_{s}^{L T P}=-\left(\frac{k_{u}+k_{l}}{G D_{s}}\right) P_{0}- \\
& \sum_{n=1}^{n=\infty}\left[\left(\frac{k_{u}+k_{l}}{G D_{s}}\right)+\left(\frac{k_{u}}{G C}+\frac{k_{l}}{G C}+\frac{1}{D_{s}}\right)\left(\frac{2 n \pi}{s}\right)^{2}+\frac{1}{C}\left(\frac{2 n \pi}{s}\right)^{4}\right] P_{n} \cos \left(\frac{2 n \pi x}{s}\right)
\end{aligned}
$$

Similarly, by substituting Eq. (11) into Eq. (10b), the following differential

equation for Region II (i.e. for $\pm r \leq x \leq \pm s / 2$ ) can be derived:

$$
\begin{aligned}
& w_{h}^{L T P^{v i}}+X_{h} w_{h}^{L T P^{i v}}+Y_{h} w_{h}^{L T P^{\prime \prime}}+Z_{h} w_{h}^{L T P}=-\left(\frac{k_{u}+k_{l}}{G D_{h}}\right) P_{0}- \\
& \sum_{n=1}^{n=\infty}\left[\left(\frac{k_{u}+k_{l}}{G D_{h}}\right)+\left(\frac{k_{u}}{G C}+\frac{k_{l}}{G C}+\frac{1}{D_{h}}\right)\left(\frac{2 n \pi}{s}\right)^{2}+\frac{1}{C}\left(\frac{2 n \pi}{s}\right)^{4}\right] P_{n} \cos \left(\frac{2 n \pi x}{s}\right)
\end{aligned}
$$

375 where

$$
\left\{\begin{array}{l}
X_{S}=-\frac{1}{G}\left(k_{u}+k_{l}+\frac{k_{u} G}{C}\right) \\
X_{h}=-\frac{1}{G}\left(k_{u}+k_{l}+\frac{k_{u} G}{C}\right)
\end{array}\right\} ;\left\{\begin{array}{l}
Y_{S}=\frac{k_{u} k_{l}}{G C}+\frac{k_{u}}{D_{s}} \\
Y_{h}=\frac{k_{u} k_{l}}{G C}+\frac{k_{u}}{D_{h}}
\end{array}\right\} ; \text { and }\left\{\begin{array}{l}
Z_{s}=-\frac{k_{u} k_{l}}{G D_{s}} \\
Z_{h}=-\frac{k_{u} k_{l}}{G D_{h}}
\end{array}\right\}
$$

The governing differential equations (i.e. Eqs. (13a) and (13b)) are sixth order, linear, and nonhomogeneous equations with constant coefficients. To obtain general 378 solutions for the governing differential equations, auxiliary or complementary 379 equations corresponding to the homogeneous equations are solved. The auxiliary equations to the homogeneous equations can be expressed in a generalised form as 381 stated in Eqs. (15a) and (15b) sourcing the solution for the original nonhomogeneous 
equations with roots $a_{s 1}$ to $a_{s 6}$ and $a_{h 1}$ to $a_{h 6}$. The auxiliary equations corresponding

383 to Eqs. (13a) and (13b) are:

$$
a_{s}^{6}+X_{s} a_{s}^{4}+Y_{s} a_{s}^{2}+Z_{s}=0, \quad-r \leq x \leq+r
$$

384 and

$$
a_{h}^{6}+X_{h} a_{h}^{4}+Y_{h} a_{h}^{2}+Z_{h}=0, \quad \pm r \leq x \leq \pm \frac{s}{2}
$$

For the sake of paper length, detailed calculation steps for the sagging section are explained in details and readers can simply use the same method to obtain the solution for the hogging region. Eq. (15a) is a polynomial equation of degree 6. Therefore, Eq. (15a) has 6 real and/or complex roots (not necessarily distinct). Considering $a_{s}{ }^{2}=\mu_{s}$, the following relation is obtained from Eq. (15a):

$$
\mu_{s}^{3}+X_{s} \mu_{s}^{2}+Y_{s} \mu_{s}+Z_{s}=0
$$

$$
b_{s}^{3}+3 \alpha_{s} b_{s}+2 \beta_{s}=0
$$

391 where

$$
\alpha_{s}=\frac{1}{3}\left(Y_{s}-\frac{X_{s}{ }^{2}}{3}\right) \text { and } \beta_{s}=\frac{1}{2}\left(\frac{2 X_{s}{ }^{3}}{27}-\frac{X_{S} Y_{S}}{3}+Z_{s}\right)
$$

$$
\Delta_{s}=-108\left(\alpha_{s}^{3}+\beta_{s}^{2}\right)
$$

It is well established in the literature (Avramidis and Morfidis, 2006; Morfidis, 2007) that the most common solution case corresponding to the positive sign of the auxiliary parameter $\Delta_{s}$ is when $\Delta_{s}<0$. Thus Eq. (19) converts to $\alpha_{s}{ }^{3}+\beta_{s}{ }^{2}>0$ with one real and two conjugate complex roots. The real root $\left(\mu_{s 1}\right)$ is as following:

$$
\mu_{s 1}=-\frac{X_{s}}{3}+\sqrt[3]{-\beta_{s}+\sqrt{\Delta_{s}}}+\sqrt[3]{-\beta_{s}-\sqrt{\Delta_{s}}}
$$

398 and the two complex roots $\left(\mu_{s 2}\right.$ and $\left.\mu_{s 3}\right)$ are as below: 


$$
\begin{aligned}
& \mu_{s 2}=-\frac{X_{s}}{3}-\frac{1}{2}\left(\sqrt[3]{-\beta_{s}+\sqrt{\Delta_{s}}}+\sqrt[3]{-\beta_{s}-\sqrt{\Delta_{s}}}\right)+i \frac{\sqrt{3}}{2}\left(\sqrt[3]{-\beta_{s}+\sqrt{\Delta_{s}}}-\right. \\
& \left.\sqrt[3]{-\beta_{s}-\sqrt{\Delta_{s}}}\right)
\end{aligned}
$$

399 and

$$
\begin{aligned}
& \mu_{s 3}=-\frac{X_{s}}{3}-\frac{1}{2}\left(\sqrt[3]{-\beta_{s}+\sqrt{\Delta_{s}}}+\sqrt[3]{-\beta_{s}-\sqrt{\Delta_{s}}}\right)-i \frac{\sqrt{3}}{2}\left(\sqrt[3]{-\beta_{s}+\sqrt{\Delta_{s}}}-\right. \\
& \left.\sqrt[3]{-\beta_{s}-\sqrt{\Delta_{s}}}\right)
\end{aligned}
$$

400 If six roots of Eq. (15a) are known as $a_{s j}$ where $j=1-6$, then the solution of the 401 homogeneous equation (Eq. (15a)) can be tabulated as:

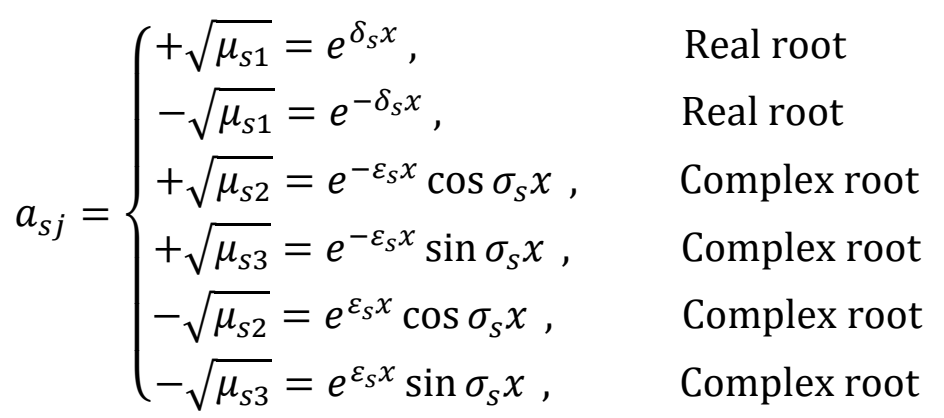

402 where

$$
\left\{\begin{array}{l}
\delta_{s}= \pm \sqrt{-\frac{X_{s}}{3}+\sqrt[3]{-\beta_{s}+\sqrt{\Delta_{s}}}+\sqrt[3]{-\beta_{s}-\sqrt{\Delta_{s}}}} \\
\varepsilon_{s}=\sqrt{\frac{1}{2}\left(\sqrt{m_{s}^{2}+n_{s}^{2}}+m_{s}\right)} \\
\sigma_{s}=\sqrt{\frac{1}{2}\left(\sqrt{m_{s}^{2}+n_{s}^{2}}-m_{s}\right)}
\end{array}\right.
$$

403 Following equations can be used to obtain $m_{s}$ and $n_{s}$ required in Eq. (22).

$$
m_{s}=-\frac{1}{2}\left(\frac{2 X_{s}}{3}+\sqrt[3]{-\beta_{s}+\sqrt{\Delta_{s}}}+\sqrt[3]{-\beta_{s}-\sqrt{\Delta_{s}}}\right)
$$

404 and

$$
n_{s}=\frac{\sqrt{3}}{2}\left(\sqrt[3]{-\beta_{S}+\sqrt{\Delta_{s}}}-\sqrt[3]{-\beta_{S}-\sqrt{\Delta_{S}}}\right)
$$


406 solutions $\left(y_{p}\right)$ must be found. Thus, trial forms for the particular integral are assumed

407 for the two differential equations with different constants which are presented in 408 Eqs.(24a) and (24b).

$$
y_{p}= \begin{cases}y_{p s}=W_{s} \cos \left(\frac{2 n \pi x}{s}\right), & -r \leq x \leq+r \\ y_{p h}=W_{h} \cos \left(\frac{2 n \pi x}{s}\right), & \pm r \leq x \leq \pm \frac{s}{2}\end{cases}
$$

409 where $W_{s}$ and $W_{h}$ are the arbitrary constants for the sagging and hogging regions,

410 respectively. These trial functions are then substituted into the corresponding 411 differential equations (i.e. Eqs. (13a) and (13b)) and the constants resulting in particular 412 solutions are obtained. Subsequently, the following expressions are obtained for the 413 particular solutions:

$$
y_{p}= \begin{cases}y_{p s}=\left(\frac{k_{u}+k_{l}}{k_{u} k_{l}}\right) P_{0}+\sum_{n=1}^{n=\infty} p_{n s} \cos \left(\frac{2 n \pi x}{s}\right), & -r \leq x \leq+r \\ y_{p h}=\left(\frac{k_{u}+k_{l}}{k_{u} k_{l}}\right) P_{0}+\sum_{n=1}^{n=\infty} p_{n h} \cos \left(\frac{2 n \pi x}{s}\right), & \pm r \leq x \leq \pm \frac{s}{2}\end{cases}
$$

414 where

$$
p_{n s}=\frac{P_{n}\left[\frac{1}{k_{u}}\left(\frac{2 n \pi}{s}\right)^{4}+\frac{k_{u}}{G D_{s}}\left(\frac{D_{s}}{C}+\frac{D_{s} k_{l}}{k_{u} C}+\frac{G}{k_{u}}\right)\left(\frac{2 n \pi}{s}\right)^{2}+\frac{\left(k_{u}+k_{l}\right)}{G D_{s}}\right]}{\left(\frac{2 n \pi}{s}\right)^{6}+\frac{1}{G}\left(k_{u}+k_{l}+\frac{k_{u} G D_{s}}{C}\right)\left(\frac{2 n \pi}{s}\right)^{4}+\frac{k_{u}}{D_{s}}\left(1+\frac{k_{l} D_{s}}{G C}\right)\left(\frac{2 n \pi}{s}\right)^{2}+\frac{k_{u} k_{l}}{G D_{s}}}
$$

415 and

$$
p_{n h}=\frac{P_{n}\left[\frac{1}{k_{u}}\left(\frac{2 n \pi}{s}\right)^{4}+\frac{k_{u}}{G D_{h}}\left(\frac{D_{h}}{C}+\frac{D_{h} k_{u}}{k_{1} C}+\frac{G}{k_{u}}\right)\left(\frac{2 n \pi}{s}\right)^{2}+\frac{\left(k_{u}+k_{l}\right)}{G D_{h}}\right]}{\left(\frac{2 n \pi}{s}\right)^{6}+\frac{1}{G}\left(k_{u}+k_{l}+\frac{k_{u} G D_{h}}{C}\right)\left(\frac{2 n \pi}{s}\right)^{4}+\frac{k_{u}}{D_{h}}\left(1+\frac{k_{l} D_{h}}{G C}\right)\left(\frac{2 n \pi}{s}\right)^{2}+\frac{k_{u} k_{l}}{G D_{h}}}
$$

416 Finally, using the superposition principle, the solution of the governing differential 417 equation (i.e. Eq. (13a)) for the settlement of the LTP with symmetric loading in the 418 sagging region (i.e. for $-r \leq x \leq+r$ ) can be written as follows: 


$$
\begin{aligned}
& w_{s}^{L T P}=c_{1} e^{-\delta_{s} x}+c_{2} e^{\delta_{s} x}+e^{-\varepsilon_{s} x}\left(c_{3} \cos \sigma_{s} x+c_{4} \sin \sigma_{s} x\right)+ \\
& e^{\varepsilon_{s} x}\left(c_{5} \cos \sigma_{s} x+c_{6} \sin \sigma_{s} x\right)+\left(\frac{k_{u}+k_{l}}{k_{u} k_{l}}\right) P_{0}+\sum_{n=1}^{n=\infty} p_{n s} \cos \left(\frac{2 n \pi x}{s}\right)
\end{aligned}
$$

Similarly, the solution of the governing differential equation for the deflection of 420 the LTP with symmetric loading in the hogging region (i.e. for $\pm r \leq x \leq \pm s / 2$ ) is 421 given by:

$$
\begin{aligned}
& w_{h}^{L T P}=d_{1} e^{-\delta_{h} x}+d_{2} e^{\delta_{h} x}+e^{-\varepsilon_{h} x}\left(d_{3} \cos \sigma_{h} x+d_{4} \sin \sigma_{h} x\right)+ \\
& e^{\varepsilon_{h} x}\left(d_{5} \cos \sigma_{h} x+d_{6} \sin \sigma_{h} x\right)+\left(\frac{k_{\mathrm{u}}+k_{1}}{k_{\mathrm{u}} k_{\mathrm{l}}}\right) P_{0}+\sum_{n=1}^{n=\infty} p_{n h} \cos \left(\frac{2 n \pi x}{s}\right)
\end{aligned}
$$

422 where $\delta_{h}, \varepsilon_{h}$, and $\sigma_{h}$ for the hogging section can be calculated following the similar 423 procedures as described for the sagging region in Eqs. (22) and (23a). Once the 424 deflections of LTP at different locations are obtained using Eqs. (27a) and (27b), the 425 rotational angles of cross sections of LTP, the shear forces generated in LTP, the 426 bending moments developed in LTP, and the tension mobilised in the geosynthetic 427 reinforcement for each section can be obtained as set out in the following sections.

428 Deflection of the shear layer embedded in the Kerr foundation can be expressed in 429 terms of $w_{L T P}$. According to Eqs. (4a) and (5a):

$$
q_{s}=k_{u}\left(w_{s}^{L T P}-w_{s}^{l s}\right), \quad-r \leq x \leq+r
$$

430 Then combination of Eqs. (28) and (9a) yields the following equation.

$$
\begin{aligned}
& w_{S}^{l s}=U_{1} w_{S}^{L T P^{i v}}-\left(\frac{U_{1} k_{u}}{C}\right) w_{S}^{L T P^{\prime \prime}}+\left(\frac{U_{1} k_{u} k_{l}}{C G}+1\right) w_{S}^{L T P}-\left(\frac{U_{1}}{D_{S}}\right) p+ \\
& \left(\frac{U_{1}}{C}\right) p^{\prime \prime}, \quad-r \leq x \leq+r
\end{aligned}
$$

431 Similarly, for the hogging region, deflection of the shear layer within the Kerr 432 foundation is given by:

$$
\begin{aligned}
& w_{h}^{l s}=U_{2} w_{h}^{L T P^{i v}}-\left(\frac{U_{2} k_{u}}{C}\right) w_{h}^{L T P^{\prime \prime}}+\left(\frac{U_{2} k_{u} k_{l}}{C G}+1\right) w_{h}^{L T P}-\left(\frac{U_{2}}{D_{h}}\right) p+ \\
& \left(\frac{U_{2}}{C}\right) p^{\prime \prime}, \quad \pm r \leq x \leq \pm \frac{s}{2}
\end{aligned}
$$


where

$$
U_{1}=\frac{D_{s} C G}{k_{u}\left[C G-D_{s}\left(k_{u}+k_{l}\right)\right]} \text { and } U_{2}=\frac{D_{h} C G}{k_{u}\left[C G-D_{h}\left(k_{u}+k_{l}\right)\right]}
$$

\subsection{Rotation of LTP}

According to the direction of bending moment (i.e. sagging or hogging), the rotation of the cross section of LTP (reinforced Timoshenko beam model) on the Kerr foundation model is given by:

$$
\theta_{L T P}=\left\{\begin{array}{c}
\theta_{s}^{L T P}=\frac{D_{s}}{C} w_{s}^{L T P^{\prime \prime \prime}}+w_{s}^{L T P^{\prime}}-\frac{D_{s}}{C^{2}} q_{s}{ }^{\prime}+\frac{D_{s}}{C^{2}} p^{\prime},-r \leq x \leq+r \\
\theta_{h}^{L T P}=\frac{D_{h}}{C} w_{h}^{L T P^{\prime \prime \prime}}+w_{h}^{L T P^{\prime}}-\frac{D_{h}}{C^{2}} q_{h}{ }^{\prime}+\frac{D_{h}}{C^{2}} p^{\prime}, \pm r \leq x \leq \pm \frac{s}{2}
\end{array}\right.
$$

Substituting Eqs. (5a) and (11) into Eq. (31a) and then utilising Eq. (27a) lead to the governing equation for rotation of the cross section of LTP in sagging region which is written below.

$$
\begin{aligned}
& \theta_{s}^{L T P}=-c_{1} A_{1} \delta_{s} e^{-\delta_{s} x}+c_{2} A_{1} \delta_{s} \mathrm{e}^{\delta_{s} \mathrm{x}}-\mathrm{c}_{3} \mathrm{e}^{-\varepsilon_{s} \mathrm{x}}\left(B_{1} \sin \sigma_{s} x-C_{1} \cos \sigma_{s} x\right)+ \\
& c_{4} e^{-\varepsilon_{S} x}\left(C_{1} \sin \sigma_{s} x+B_{1} \cos \sigma_{s} x\right)-c_{5} e^{\varepsilon_{s} x}\left(B_{1} \sin \sigma_{s} x+C_{1} \cos \sigma_{s} x\right)- \\
& c_{6} e^{\varepsilon_{S} x}\left(C_{1} \sin \sigma_{s} x-B_{1} \cos \sigma_{s} x\right)-\sum_{n=1}^{n=\infty}\left\{\left[D_{1}+E_{1}\left(\frac{2 n \pi}{s}\right)^{4}-\right.\right. \\
& \left.\left.F_{1}\left(\frac{2 n \pi}{s}\right)^{2}\right] p_{n s}+\left[\left(\frac{G F_{1} D_{s}{ }^{2}}{C^{2}}\right)\left(\frac{2 n \pi}{s}\right)^{2}+\left[\left(\frac{G F_{1} D_{s}}{C}\right)+\frac{D_{s}}{C^{2}}\right]\right] P_{n}\right\}\left(\frac{2 n \pi}{s}\right) \sin \left(\frac{2 n \pi x}{s}\right)
\end{aligned}
$$

In the same way, combining Eqs. (5b), (11), (27b), and (31b), the governing equation for rotation of the cross section of LTP in hogging region can be expressed as:

$$
\begin{aligned}
& \theta_{h}^{L T P}=-d_{1} A_{2} \delta_{h} e^{-\delta_{h} x}+d_{2} A_{2} \delta_{h} e^{\delta_{h} x}-d_{3} e^{-\varepsilon_{h} x}\left(B_{2} \sin \sigma_{h} x-\right. \\
& \left.C_{2} \cos \sigma_{h} x\right)+d_{4} e^{-\varepsilon_{h} x}\left(C_{2} \sin \sigma_{h} x+B_{2} \cos \sigma_{h} x\right)-d_{5} e^{\varepsilon_{h} x}\left(B_{2} \sin \sigma_{h} x+\right. \\
& \left.C_{2} \cos \sigma_{h} x\right)-d_{6} e^{\varepsilon_{h} x}\left(C_{2} \sin \sigma_{h} x-B_{2} \cos \sigma_{h} x\right)-\sum_{n=1}^{n=\infty}\left\{\left[D_{2}+\right.\right. \\
& \left.E_{2}\left(\frac{2 n \pi}{s}\right)^{4}-F_{2}\left(\frac{2 n \pi}{s}\right)^{2}\right] p_{n h}+\left[\left(\frac{G F_{2} D_{h}^{2}}{C^{2}}\right)\left(\frac{2 n \pi}{s}\right)^{2}+\left[\left(\frac{G F_{2} D_{h}}{C}\right)+\right.\right.
\end{aligned}
$$




$$
\left.\left.\left.\frac{D_{h}}{C^{2}}\right]\right] P_{n}\right\}\left(\frac{2 n \pi}{s}\right) \sin \left(\frac{2 n \pi x}{s}\right)
$$

443 where

$$
\begin{aligned}
& \left\{\begin{array}{l}
A_{1}=\delta_{s}\left(\delta_{s}{ }^{4} E_{1}+\delta_{s}{ }^{2} F_{1}+D_{1}\right) \\
A_{2}=\delta_{h}\left(\delta_{h}{ }^{4} E_{2}+\delta_{h}{ }^{2} F_{2}+D_{2}\right)
\end{array}\right\} \\
& \left\{\begin{array}{c}
B_{1}=\sigma_{s}\left[E_{1}\left(\sigma_{s}{ }^{4}-10 \varepsilon_{s}{ }^{2} \sigma_{s}{ }^{2}+5 \varepsilon_{s}{ }^{4}\right)+F_{1}\left(3 \varepsilon_{s}{ }^{2}-\sigma_{s}{ }^{2}\right)+D_{1}\right] \\
B_{2}=\sigma_{h}\left[E_{2}\left(\sigma_{h}{ }^{4}-10 \varepsilon_{h}{ }^{2} \sigma_{h}{ }^{2}+5 \varepsilon_{h}{ }^{4}\right)+F_{2}\left(3 \varepsilon_{h}{ }^{2}-\sigma_{h}{ }^{2}\right)+D_{2}\right]
\end{array}\right\} \\
& \left\{\begin{array}{l}
C_{1}=-\varepsilon_{s}\left[E_{1}\left(\varepsilon_{s}{ }^{4}-10 \varepsilon_{s}{ }^{2} \sigma_{s}{ }^{2}+5 \sigma_{s}{ }^{4}\right)+F_{1}\left(\varepsilon_{s}{ }^{2}-3 \sigma_{s}{ }^{2}\right)+D_{1}\right] \\
C_{2}=-\varepsilon_{h}\left[E_{2}\left(\varepsilon_{h}{ }^{4}-10 \varepsilon_{h}{ }^{2} \sigma_{h}{ }^{2}+5 \sigma_{h}{ }^{4}\right)+F_{2}\left(\varepsilon_{h}{ }^{2}-3 \sigma_{h}{ }^{2}\right)+D_{2}\right]
\end{array}\right\} \\
& \left\{\begin{array}{l}
D_{1}=1-\left(\frac{k_{u} k_{l} G_{1} D_{s}{ }^{2}}{C^{2}}\right) \\
D_{2}=1-\left(\frac{k_{u} k_{l} G_{2} D_{h}{ }^{2}}{C^{2}}\right)
\end{array}\right\} \\
& \left\{\begin{array}{l}
\left.E_{1}=-\frac{G G_{1} D_{s}{ }^{2}}{C}\right) \\
\left.E_{2}=-\frac{G G_{2} D_{h}{ }^{2}}{C}\right)
\end{array}\right. \\
& \left\{\begin{array}{l}
F_{1}=\frac{D_{s}}{C}\left(1+\frac{G k_{u} G_{1} D_{s}}{C}\right) \\
F_{2}=\frac{D_{h}}{C}\left(1+\frac{G k_{u} G_{2} D_{h}}{C}\right)
\end{array}\right\}
\end{aligned}
$$

444 and

$$
\left\{\begin{array}{l}
G_{1}=\frac{D_{s}}{C}-\frac{D_{s}}{C^{2}} \frac{G k_{u}}{k_{u}+k_{l}} \\
G_{2}=\frac{D_{h}}{C}-\frac{D_{h}}{C^{2}} \frac{G k_{u}}{k_{u}+k_{l}}
\end{array}\right\}
$$

\subsection{Bending moment and shear force in LTP}

According to the theory of Timoshenko beam (1921), the relationship between

447 moment and the rate of rotation angle change can be written as:

$$
M_{L T P}=\left\{\begin{array}{l}
M_{S}^{L T P}=-D_{s} \theta_{s}^{L T P^{\prime}},-r \leq x \leq+r \\
M_{h}^{L T P}=-D_{h} \theta_{h}^{L T P^{\prime}}, \pm r \leq x \leq \pm \frac{s}{2}
\end{array}\right.
$$




$$
\begin{aligned}
& M_{s}^{L T P}=-D_{s}\left\{c_{1} A_{1} \delta_{s}{ }^{2} e^{-\delta_{s} x}+c_{2} A_{1} \delta_{s}{ }^{2} e^{\delta_{s} x}+c_{3} e^{-\varepsilon_{s} x}\left(J_{1} \sin \sigma_{s} x-\right.\right. \\
& \left.I_{1} \cos \sigma_{s} x\right)-c_{4} e^{-\varepsilon_{s} x}\left(I_{1} \sin \sigma_{s} x+J_{1} \cos \sigma_{s} x\right)-c_{5} e^{\varepsilon_{s} x}\left(J_{1} \sin \sigma_{s} x+\right. \\
& \left.I_{1} \cos \sigma_{s} x\right)-c_{6} e^{\varepsilon_{s} x}\left(I_{1} \sin \sigma_{s} x-J_{1} \cos \sigma_{s} x\right)-\sum_{n=1}^{n=\infty}\left\{\left[D_{1}+E_{1}\left(\frac{2 n \pi}{s}\right)^{4}-\right.\right. \\
& \left.\left.\left.F_{1}\left(\frac{2 n \pi}{s}\right)^{2}\right] p_{n s}+\left[\left(\frac{G F_{1} D_{s}^{2}}{C^{2}}\right)\left(\frac{2 n \pi}{s}\right)^{2}+\left[\left(\frac{G F_{1} D_{s}}{c}\right)+\frac{D_{s}}{C^{2}}\right]\right] P_{n}\right\}\left(\frac{2 n \pi}{s}\right)^{2} \cos \left(\frac{2 n \pi x}{s}\right)\right\}
\end{aligned}
$$

450 The following can be derived from Eqs. (32b) and (34b):

$$
\begin{aligned}
& M_{h}^{L T P}=-D_{h}\left\{d_{1} A_{2} \delta_{h}{ }^{2} e^{-\delta_{2} x}+d_{2} A_{2} \delta_{h}{ }^{2} e^{\delta_{h} x}+d_{3} e^{-\varepsilon_{h} x}\left(J_{2} \sin \sigma_{h} x-\right.\right. \\
& \left.I_{2} \cos \sigma_{h} x\right)-d_{4} e^{-\varepsilon_{h} x}\left(I_{2} \sin \sigma_{h} x+J_{2} \cos \sigma_{h} x\right)-d_{5} e^{\varepsilon_{h} x}\left(J_{2} \sin \sigma_{h} x+\right. \\
& \left.I_{2} \cos \sigma_{h} x\right)-d_{6} e^{\varepsilon_{h} x}\left(I_{2} \sin \sigma_{h} x-J_{2} \cos \sigma_{h} x\right)-\sum_{n=1}^{n=\infty}\left\{\left[D_{2}+E_{2}\left(\frac{2 n \pi}{s}\right)^{4}-\right.\right. \\
& \left.F_{2}\left(\frac{2 n \pi}{s}\right)^{2}\right] p_{n h}+\left[\left(\frac{G F_{2} D_{h}{ }^{2}}{C^{2}}\right)\left(\frac{2 n \pi}{s}\right)^{2}+\left[\left(\frac{G F_{2} D_{h}}{C}\right)+\right.\right. \\
& \left.\left.\left.\left.\frac{D_{h}}{C^{2}}\right]\right] P_{n}\right\}\left(\frac{2 n \pi}{s}\right)^{2} \cos \left(\frac{2 n \pi x}{s}\right)\right\}
\end{aligned}
$$

451 According to the direction of bending moment (i.e. sagging or hogging) the shear 452 force in LTP can be expressed as:

$$
V_{L T P}=\left\{\begin{array}{l}
V_{s}^{L T P}=C\left(w_{s}^{L T P^{\prime}}-\theta_{s}^{L T P}\right),-r \leq x \leq+r \\
V_{h}^{L T P}=C\left(w_{h}^{L T P^{\prime}}-\theta_{h}^{L T P}\right), \quad \pm r \leq x \leq \pm \frac{s}{2}
\end{array}\right.
$$

453 By substituting Eqs. (27a) and (32a) into Eq.(36a), the shear forces developed in 454 the LTP can be obtained as:

$$
\begin{aligned}
& V_{s}^{L T P}=C\left\{c_{1} K_{1} \delta_{s} e^{-\delta_{s} x}-c_{2} K_{1} \delta_{s} e^{\delta_{s} x}-c_{3} e^{-\varepsilon_{s} x}\left(M_{1} \sin \sigma_{s} x+L_{1} \cos \sigma_{s} x\right)-\right. \\
& c_{4} e^{-\varepsilon_{s} x}\left(L_{1} \sin \sigma_{s} x-M_{1} \cos \sigma_{s} x\right)-c_{5} e^{\varepsilon_{s} x}\left(M_{1} \sin \sigma_{s} x-L_{1} \cos \sigma_{s} x\right)+
\end{aligned}
$$




$$
\begin{aligned}
& c_{6} e^{\varepsilon_{s} x}\left(L_{1} \sin \sigma_{s} x+M_{1} \cos \sigma_{s} x\right)+\sum_{n=1}^{n=\infty}\left[D_{1}+E_{1}\left(\frac{2 n \pi}{s}\right)^{4}-F_{1}\left(\frac{2 n \pi}{s}\right)^{2}-\right. \\
& \left.1] p_{n s}+\left[\left(\frac{G F_{1} D_{s}^{2}}{C^{2}}\right)\left(\frac{2 n \pi}{s}\right)^{2}+\left[\left(\frac{G F_{1} D_{s}}{C}\right)+\frac{D_{s}}{C^{2}}\right]\right] P_{n}\left(\frac{2 n \pi}{s}\right) \sin \left(\frac{2 n \pi x}{s}\right)\right\}
\end{aligned}
$$

457 developed in the LTP in hogging region can be obtained as:

$$
\begin{aligned}
& V_{h}^{L T P}=C\left\{d_{1} K_{2} \delta_{h} e^{-\delta_{h} x}-d_{2} K_{2} \delta_{h} e^{\delta_{h} x}-d_{3} e^{-\varepsilon_{h} x}\left(M_{2} \sin \sigma_{h} x+\right.\right. \\
& \left.L_{2} \cos \sigma_{h} x\right)-d_{4} e^{-\varepsilon_{h} x}\left(L_{2} \sin \sigma_{h} x-M_{2} \cos \sigma_{h} x\right)-d_{5} e^{\varepsilon_{h} x}\left(M_{2} \sin \sigma_{h} x-\right. \\
& \left.L_{2} \cos \sigma_{h} x\right)+d_{6} e^{\varepsilon_{h} x}\left(L_{2} \sin \sigma_{h} x+M_{2} \cos \sigma_{h} x\right)+\sum_{n=1}^{n=\infty}\left[D_{2}+E_{2}\left(\frac{2 n \pi}{s}\right)^{4}-\right. \\
& \left.F_{2}\left(\frac{2 n \pi}{s}\right)^{2}-1\right] p_{n h}+\left[\left(\frac{G F_{2} D_{h}{ }^{2}}{C^{2}}\right)\left(\frac{2 n \pi}{s}\right)^{2}+\left[\left(\frac{G F_{2} D_{h}}{C}\right)+\right.\right. \\
& \left.\left.\left.\frac{D_{h}}{C^{2}}\right]\right] P_{n}\left(\frac{2 n \pi}{s}\right) \sin \left(\frac{2 n \pi x}{s}\right)\right\}
\end{aligned}
$$

458 where

$$
\begin{aligned}
& \left\{\begin{array}{l}
I_{1}=\varepsilon_{s} C_{1}+\sigma_{s} B_{1} \\
I_{2}=\varepsilon_{h} C_{2}+\sigma_{h} B_{2}
\end{array}\right\} \\
& \left\{\begin{array}{l}
J_{1}=\varepsilon_{s} B_{1}-\sigma_{s} C_{1} \\
J_{2}=\varepsilon_{h} B_{2}-\sigma_{h} C_{2}
\end{array}\right\} \\
& \left\{\begin{array}{l}
K_{1}=\delta_{s}-A_{1} \\
K_{2}=\delta_{s}-A_{2}
\end{array}\right\} \\
& \left\{\begin{array}{l}
L_{1}=\varepsilon_{s}+C_{1} \\
L_{2}=\varepsilon_{h}+C_{2}
\end{array}\right\}
\end{aligned}
$$

459 and

$$
\left\{\begin{array}{l}
M_{1}=\sigma_{s}-B_{1} \\
M_{2}=\sigma_{h}-B_{2}
\end{array}\right\}
$$

\subsection{Tension in geosynthetic reinforcement}

Tension mobilised in the geosynthetic reinforcement is the product of axial strain

462 in the geosynthetic reinforcement (which is assumed to be equal to the strain developed 
in the LTP at the location of geosynthetic reinforcement) and the tensile stiffness of the

464 geosynthetic reinforcement. Following the Timoshenko beam theory and depending on

465 the bending moment directions, the tension mobilised in the geosynthetic reinforcement

466 can be expressed as follows:

$$
T= \begin{cases}-S_{r}^{b}\left(y_{r}^{b}+y_{s}\right) \theta_{s}^{L T P^{\prime}}, & -r \leq x \leq+r \\ -S_{r}^{t}\left(y_{r}^{t}+y_{h}\right) \theta_{h}^{L T P^{\prime}}, & \pm r \leq x \leq \pm \frac{s}{2}\end{cases}
$$

467 where $y_{r}^{t}$ and $y_{r}^{b}$ are the distances from the top and bottom geosynthetic layer to the 468 centroid axis, respectively as shown in Fig. $2 \mathrm{~b} ; y_{s}$ and $y_{h}$ are the distances between 469 neutral axis and centroid axis of LTP within the sagging and hogging moment sections, 470 respectively as shown in Figs. $2 \mathrm{~b}-\mathrm{c}$; and $S_{r}^{t}$ and $S_{r}^{b}$ are the tensile stiffnesses of top and

471 bottom geosynthetic reinforcements, respectively.

\subsection{Pressure distribution under LTP}

473 Combining Eqs. (4a), (7a), and (9a), the pressure distribution under the LTP for

$474-r \leq x \leq+r$ can be obtained as below:

$$
\begin{aligned}
& q_{s}=\frac{G C D_{S}}{\left[D_{S}\left(k_{u}+k_{l}\right)-G C\right]} w_{S}^{L T P^{i v}}-\frac{k_{u} D_{S} G}{\left[D_{S}\left(k_{u}+k_{l}\right)-G C\right]} w_{S}^{L T P^{\prime \prime}}+\frac{k_{u} k_{l} D_{S}}{\left[D_{S}\left(k_{u}+k_{l}\right)-G C\right]} w_{S}^{L T P}- \\
& \frac{G C}{\left[D_{s}\left(k_{u}+k_{l}\right)-G C\right]} p+\frac{D_{S} G C}{\left[D_{s}\left(k_{u}+k_{l}\right)-G C\right]} p^{\prime \prime}
\end{aligned}
$$

475 Similarly, from Eqs. (4b), (7b), and (9b), the pressure distribution under the LTP

476 for $\pm r \leq x \leq \pm s / 2$ can be expressed as:

$$
\begin{aligned}
& q_{h}=\frac{G C D_{h}}{\left[D_{h}\left(k_{u}+k_{l}\right)-G C\right]} w_{h}^{L T P^{i v}}-\frac{k_{u} D_{h} G}{\left[D_{h}\left(k_{u}+k_{l}\right)-G C\right]} w_{h}^{L T P^{\prime \prime}}+ \\
& \frac{k_{u} k_{l} D_{h}}{\left[D_{h}\left(k_{u}+k_{l}\right)-G C\right]} w_{h}^{L T P}-\frac{G C}{\left[D_{h}\left(k_{u}+k_{l}\right)-G C\right]} p+\frac{D_{h} G C}{\left[D_{h}\left(k_{u}+k_{l}\right)-G C\right]} p^{\prime \prime}
\end{aligned}
$$




\subsection{Boundary and continuity conditions}

Referring to Eqs. (27a) and (27b), there are twelve constants of integration ( $c_{1}$ to $c_{6}$ and $d_{1}$ to $d_{6}$ ) and one unknown length $(r)$ that can be estimated using the boundary and continuity conditions. Due to symmetric loading, at the middle of loaded region, the shear force and the slope of the deflected LTP are zero. Additionally, it is presumed that at the column location, the shear force produced in LTP is equivalent to the reaction force from the column. It is also assumed here that due to inclusion of the geosynthetic reinforcement in LTP and continuity of LTP above the column, LTP will not be rotating at the column support. Summary of the above-mentioned boundary conditions are expressed in Eq. (41).

$$
\text { at } x=0,\left\{\begin{array}{l}
V_{s}^{L T P}=0 \\
w_{s}^{L T P^{\prime}}=0
\end{array} \quad \text { and } \quad \text { at } x=\frac{s}{2},\left\{\begin{array}{c}
V_{h}^{L T P}=-\left(K_{c}\right)_{e q} w_{h}^{L T P} \\
\theta_{h}^{L T P}=0
\end{array}\right.\right.
$$

where $\left(K_{c}\right)_{e q}$ is the equivalent modulus of subgrade reaction for a column in a plane strain condition $(\mathrm{kN} / \mathrm{m})$ which can be calculated as Eq. (42).

$$
\left(K_{c}\right)_{e q}=\frac{\left(E_{c}\right)_{e q}}{H_{c}} \times \frac{A_{c}}{s}
$$

where $A_{c}$ is the area of the column in plane strain condition (i.e. $A_{c}=s \times d$ ); $s$ and $d$ are the clear spacing and the diameter of the column, respectively as shown in Fig. 3a; $H_{c}$ is the length of column; and $\left(E_{c}\right)_{e q}$ is the equivalent elastic modulus of the column wall in plane strain condition. Since in the field, discrete columns are placed in a square or triangular pattern, the equivalent plane strain material stiffness must be determined for the two-dimensional plane strain modeling. In the literature, there are two approaches for plane strain equivalent conversion (Tan et al., 2008). In the first approach, the width of the column (in plane-strain condition) can be taken equal to the diameter of the column (in axisymmetric condition). However, the material stiffness in axisymmetric model should be converted to equivalent plane-strain material stiffness 
by the suggested relationship based on the matching of the column-soil composite

500 stiffness. This approach was adopted by Huang et al., (2009) where the equivalent

501 elastic modulus and cohesion of the deep mixing walls were calculated during the

502 investigation of coupled mechanical and hydraulic modelling of geosynthetic-

503 reinforced column-supported embankments. In the second approach, geometrical

504 conversion can be done to obtain similar response in both axisymmetric and plane-

505 strain conditions as adopted by Tan et al. (2008). In this study, first approach to convert

506 a 3D or axisymmetric model into an equivalent plane-strain model is adopted. The

507 equivalent modulus is calculated using the area replacement ratio as stated by Huang et

508 al. (2009) as follows:

$$
\left(E_{c}\right)_{e q}=E_{c} a_{r}+E_{s}\left(1-a_{r}\right)
$$

509 where $E_{c}$ and $E_{s}$ denote the elastic moduli of the column and soft soil, respectively; 510 while $a_{r}$ is the area replacement ratio. Similar approach (i.e. first approach) was 511 adopted by Huang et al. (2009) and Deb and Mohapatra (2013) where deep mixing 512 columns and stone columns supported embankments were analysed in plane-strain 513 condition in which the equivalent plane-strain material stiffness of column was 514 determined using the suggested relationship based on the matching of the column-soil 515 composite stiffness.

516 On the other hand, the effective cross section of the LTP in the sagging region (the 517 left side of point "A" as shown in Fig. 2a) is not the same as the hogging region (right 518 side of point "A"). Hence, the deflections and internal forces in the LTP beam should 519 be represented by two separate functions. However, the deflection curve and internal 520 forces of LTP are physically continuous at point "A" and therefore the continuity 521 conditions for the deflections and moments must be satisfied at point "A". Each of these 
continuity conditions yields to an equation for evaluating the unknowns. The continuity conditions can be summarised as below:

$$
\text { at } x=r \text { (Point "A"), }\left\{\begin{array}{c}
w_{s}^{L T P}=w_{h}^{L T P} \\
\theta_{s}^{L T P}=\theta_{h}^{L T P} \\
M_{s}^{L T P}=0 \\
M_{h}^{L T P}=0 \\
V_{s}^{L T P}=V_{h}^{L T P}
\end{array}\right.
$$
shear force of LTP and the shear layer from Eqs. (27), (32), (35), (37), and (29) respectively into the boundary and the continuity conditions (Eqs. (41) and (44)-(46))

535 yields thirteen algebraic equations which are summarised in Appendix. Once all the 536 constants of integration and unknown lengths are determined by solving the 
simultaneous equations, then the deflections, bending moments, shear forces, rotations

538 of the LTP, and mobilised tension in the geosynthetic reinforcement at any point in the

539 LTP can be determined.

540 Although the overall behaviour of LTP due to bending and shear actions on a soft

541 soil foundation can be predicted using the proposed mechanical model, it should be 542 noted that possible pull-out resistance force of geosynthetic reinforcement, 543 permeability of soft soil, and cyclic loading can significantly affect the performance of 544 soft soil (Indraratna et al., 2005, Suksiripattanapong et al., 2012, Indraratna et al., 545 2013b).

\section{Results and discussions}

Due to symmetry, only half of the problem is considered for the parametric study.

548 Based on the formulations and for the sake of convenience and practical use, all the 549 algebraic equations have been programmed in MATLAB R2016b (MathWorks) and 550 the results are presented graphically. Similar to Maheshwari and Viladkar (2009), 551 Zhang et al. (2012b), and Lei et al. (2016), to evaluate the accuracy of implementation of 552 the Kerr foundation model as the soft soil model, the response of double layer geosynthetic 553 reinforced LTP, the tension mobilised in the geosynthetic reinforcement, and stress 554 concentration ratio are compared with the results gained from the Pasternak and the 555 Winkler foundation models. Maheshwari and Viladkar (2009) developed a mechanical 556 model for geosynthetic reinforced soil-foundation system subjected to strip loading and 557 carried out a parametric study to understand the effect of various parameters influencing 558 the response of such a system without validating the proposed model with field or 559 experimental results. Zhang et al. (2012b) proposed a mechanical model of geocell mattress 560 subjected to symmetric loads and the presented solution was verified through comparison 561 with the other existing published solutions namely Zhang et al. (2010) and Qu (2009). Lei 
et al. (2016) derived an analytical solution to predict consolidation with vertical drains under impeded drainage boundary conditions and multi-ramp surcharge loading. To verify the validity and accuracy of the proposed analytical solution, the results calculated from the proposed solution were compared to those given by the analytical solution of Gray (1944). As far as the maximum settlement of LTP and tension mobilised in the geosynthetic reinforcement (GR) are concerned, the parametric studies have been carried out to show the effects of various parameters on the maximum settlement of LTP and tension mobilised in the geosynthetic reinforcement when the soft soil is idealised by the Kerr foundation model. In this study, mobilised tension in the reinforcement is expressed as a normalised form $\left(T / T_{y}\right)$ assuming ultimate or yield strength of geosynthetic reinforcement is $10 \%$ of tensile stiffness of geosynthetic (i.e. $T_{y}=10 \% \times S_{r}$ ). Additionally, the results of a double layer geosynthetic reinforced granular fill are compared with a single layer geosynthetic reinforced granular fill. Most of the guidelines adopt single layer of geosynthetics, whereas in practice, it is often common to use two or three layers of geosynthetics. However, to reduce the thickness of LTP, use of single layer but stronger geosynthetic reinforcement may be a good option. Thus, the intention of this parametric study is to investigate whether the use of one stronger geosynthetic layer (e.g. $1 \times 2000 \mathrm{kN} / \mathrm{m}$ ) with the equivalent stiffness of two weaker geosynthetic layers (e.g. $2 \times 1000 \mathrm{kN} / \mathrm{m}$ ), results in the same settlement of LTP and the tension of the geosynthetic reinforcement when compared to two weaker geosynthetic layers or not. For the sake of reasonable comparison, similar overall tensile stiffness due to the geosynthetic layers is adopted. For example, $2 \times 1000 \mathrm{kN} / \mathrm{m}$ tensile stiffness of geosynthetics for the double layer is compared with $1 \times 2000 \mathrm{kN} / \mathrm{m}$ tensile stiffness of a single layer geosynthetics. For two layers' case, geosynthetic reinforcement is placed such that the reinforcement layers equally divide the granular fill layer while the 
one layer of geosynthetic layer is simply placed at the centre of granular layer for the single layer case. It has been noticed in the literature that many researchers placed the single layer of geosynthetic reinforcement at the mid-level of LTP in their studies (Liu et al., 2007; Nunez et al., 2013). However, it should be noted that geosynthetics can be

591 placed at any level of LTP in case of single layer analysis in the proposed mechanical 592 model. For practical application purposes, the spring constants and the shear modulus 593 of soft soil can be estimated following the procedures proposed by Jones and 594 Xenophontos (1976) for the Kerr foundation model which are summarised as below:

$$
\begin{aligned}
& k_{u}=\frac{E_{1}\left(1-v_{1}\right)}{h_{1}\left(1-v_{1}-2 v_{1}^{2}\right)} ; k_{l}=\frac{E_{2} \gamma\left(1-v_{2}\right)\left(\sinh \gamma h_{2} \cosh \gamma h_{2}+\gamma h_{2}\right)}{2\left(1-v_{2}-2 v_{2}^{2}\right) \sinh ^{2} \gamma h_{2}} ; \text { and } \\
& G=\frac{E_{2}\left(\sinh \gamma h_{2} \cosh \gamma h_{2}-\gamma h_{2}\right)}{4 \gamma\left(1+v_{2}\right) \sinh ^{2} \gamma h_{2}}
\end{aligned}
$$

595 where Jones and Xenophontos (1976) assumed a foundation consisting of two layers 596 with elastic coefficients $\left(E_{1}, v_{1}\right)$ and $\left(E_{2}, v_{2}\right)$ and thicknesses $h_{1}$ and $h_{2}$ as illustrated 597 in Fig. 1a, respectively. The term $\gamma$ is a constant, governing the vertical deformation 598 profile. In this study, it is assumed that $\gamma=0.46$ at the mid-depth of the second layer 599 with thickness $h_{2}$ as Kneifati (1985) assumed in his study. Since the analytical solution 600 for homogeneous soil deposit is obtained for one layer only (i.e. $H=10 \mathrm{~m}$ ), and in 601 order to determine the corresponding parameters for the Kerr foundation, (see Eq. (47)), 602 it is assumed that $h_{1}=1 \mathrm{~m} ; h_{2}=9 \mathrm{~m} ; E_{1}=E_{2}=E_{s}=1000 \mathrm{kPa} ; v_{1}=v_{2}=v_{s}=0.3$. 603 Following the Kerr foundation model, it is presumed that the upper layer of soft soil experiences significant shear deformations (exceeding the shear strength of the soft soil) as commonly modelled by the Winkler foundation. While the lower layer in Kerr

606 foundation model is subjected to both compressive and shear stresses without 607 exceeding the shear strength. Therefore, $h_{1}$ and $h_{2}$ have been selected in such a way 608 that the maximum shear stress generated in the top section of the soft soil $\left(h_{1}\right)$ reaches 
the shear strength of the soil, while the shear strength of the soft soil is not exceeded in the bottom part $\left(h_{2}\right)$. It has been noticed that decreasing the depth of upper layer results in larger shear stresses generated in the bottom part of the soft soil $\left(h_{2}\right)$ which exceeds the shear strength of the soft soil. The foregoing solution is evaluated for a uniform load of $200 \mathrm{kPa}$ which includes the self-weight of LTP. The proposed analytical model is a generalised model to analyse the ground stabilised using columns (such as controlled modulus columns, piles, deep soil mixing columns) where load transfer platform is used to enhance the distribution of the load from the super-structures (such as silos, and fuel tanks) to the columns. However, typical properties of controlled modulus columns (CMCs) from a real project in Australia (Highway upgrade, approximately $100 \mathrm{~km}$ south of Sydney), is adopted in this study. The material properties used in this study for the baseline case are summarised in Table 1. For the parametric study, one parameter is changed at one time to investigate the influence of that particular parameter. The adopted range of the parameters for the parametric study summarised in Table 2 is considered to cover the typical ranges observed in real projects for the column improved soft soil. In addition, the calculated LTP parameters for double and single layer cases for the baseline case are summarised in Table 3.

\subsection{Predictions of Kerr foundation versus other foundation models}

In order to verify the validity and accuracy of the proposed analytical solution, the results calculated from the proposed solution for load transfer platform are compared with those given by the analytical solution of the same LTP resting on the Winkler (1867) and the Pasternak (1954) foundations. It is noted that when the shear modulus is equal to zero (i.e. $G=0$ ), Eqs. (10a) and (10b) reduce to fourth-order governing differential equations which simulates the response of LTP on Winkler foundation model. Additionally, when the upper spring modulus approaches infinite (i.e. $k_{u} \rightarrow \infty$ ), 
634 Eqs. (10a) and (10b) are reduced to a fourth-order governing differential equations of 635 the LTP on Pasternak foundation model. For the Winkler model, according to Horvath $636 \quad(1983)$

$$
k_{w}=\frac{E_{S}}{H}
$$

For the Pasternak model, according to Kerr (1964)

$$
k_{p}=\frac{E_{S}}{H} \text { and } G_{p}=\frac{E_{S} H}{6\left(1+v_{S}\right)}
$$

Fig. 3a shows a comparison of the deflection of the LTP adopting the Kerr

639 foundation model to simulate the soft soil against the Winkler and the Pasternak 640 models. There are notable variations in the predictions considering different foundation 641 models. As evident, adopting the Winkler foundation model results in larger deflection 642 of LTP compared to the Kerr foundation model. In contrast, Pasternak model results in 643 less deflection of LTP than the Kerr foundation model. For example, the maximum 644 deflection of LTP adopting the soft soil as Winkler foundation model is about $29 \mathrm{~mm}$, 645 while in Kerr foundation model case the value drops to $25 \mathrm{~mm}$, shown in Fig. 3a. 646 Winkler model only considers the compressibility of the soft soil without any shear 647 resistance. Therefore, the soft soil which is idealised by the Winkler foundation model 648 is prone to an excessive settlement resulting in the largest deformation of the LTP. In 649 contrast, Pasternak foundation model predicts the maximum deflection of LTP of 18 $650 \mathrm{~mm}$, which is $28 \%$ less than the corresponding value from the Kerr foundation model 651 as given in Fig. 3a. Since the Pasternak shear layer beneath the LTP is a continuous 652 layer deforming based on elastic shear only, minimum settlement of soil and 653 consequently LTP is occurred. In case of the soft soil idealised by the Kerr foundation, 654 the soil just below the LTP (from the ground surface up to $h_{1}$ ) deforms due to the 655 compressibility of the soft soil only, while in deeper areas both shear resistance and compressibility of the soft soil are contributing to the deformation. Therefore, soft soil 
simulated with the Kerr foundation behaves stiffer than the Winkler foundation while being softer than the Pasternak foundation. Hence, the Kerr foundation model predicts the deformations more realistically between two upper and lower bounds which are the Winkler and the Pasternak foundation models, respectively.

Fig. $3 b$ shows the predictions of the variation of the rotations of the LTP adopting the soft soil as Kerr, Winkler, and Pasternak foundation models. It is noticed that the Winkler foundation predicts larger LTP rotation compared to the Kerr foundation model. In contrast, the Pasternak model calculates less rotation of LTP compared to the Kerr model. For example, the maximum rotation of LTP when the Kerr foundation model is adopted for the soft soil is -0.03 radians, which increases to -0.04 radians for the Winkler foundation model (i.e. 33\% increase) and decreases to -0.019 radians for the Pasternak foundation model (i.e. 37\% decrease) as displayed in Fig. 3b. This is since implementing the Winkler model predicts the largest deformation of the LTP (see Fig. 3a); hence the largest rotation of LTP is achieved in the Winkler model. In contrast, adopting the Pasternak model predicts the smallest deformation of LTP (see Fig. 3a), it results in the least rotation of LTP. Accordingly, the Kerr foundation model predicts the rotations more precisely which is between two upper and lower bounds corresponding to the Winkler and the Pasternak foundation models, respectively.

$$
\text { In Fig. 4a, the distribution of the bending moment along the length of the LTP is }
$$
presented. It is observed that the maximum positive and negative moments in the LTP adopting the Winkler foundation model are approximately $6 \%$ and $12 \%$ more, respectively, than the corresponding values when the Kerr foundation model is used to simulate the soft soil. In contrast, Pasternak model predicts smaller positive (sagging) and negative (hogging) bending moments in the LTP compared to the Kerr foundation model. As an illustration, the Pasternak foundation model estimates the maximum 
positive and negative moments in the LTP approximately $35 \%$ and $21 \%$ less than the corresponding values when the Kerr foundation model is used to simulate the soft soil, respectively, as illustrated in Fig. 4a. Referring to Fig. 3a, since implementing the Winkler model results in the largest deformation of the LTP, the largest moments in the LTP are developed correspondingly. In contrast, the Pasternak model predicts the smallest deformation of LTP (see Fig. 3a), hence it predicts the least moments in the LTP. Accordingly, similar to the deformations reported, the Kerr foundation model calculates the moments more accurately, which are between the upper (i.e. Winkler foundation) and lower bounds(i.e. Pasternak foundation) .

Fig. $4 \mathrm{~b}$ shows a comparison of the shear forces developed in the LTP using the

692 Kerr foundation model to pretend the soft soil against the Winkler and the Pasternak foundation models. From Fig. $4 \mathrm{~b}$ it is depicted that the Winkler model estimates larger shear force in LTP as compared to the Kerr model. Whereas, the Pasternak model predicts less shear force in the LTP incomparision to the Kerr model. For example, the maximum shear force in LTP adopting the Kerr foundation model is $131 \mathrm{kN} / \mathrm{m}$, which increases to $140 \mathrm{kN} / \mathrm{m}$ and reduces to $128 \mathrm{kN} / \mathrm{m}$ in the Winkler and the Pasternak foundation models, respectively. Since adopting the Winkler model predicts larger deflection of LTP compared to the Kerr model (refer to Fig. 3a), shear force induced in the LTP is also greater. On the other hand, adopting the Pasternak model predicts less

701 deflection of LTP incomparision to the Kerr model (see Fig. 3a); hence predicted shear 702 force induced in LTP is also smaller.

Fig. $4 \mathrm{c}$ represents the variation of shear forces developed in the soft soil between two columns. As expected, at the mid span, the shear force in the soil is zero due to the

705 symmetric condition while the Kerr and the Pasternak foundation models are used to 706 idealise the soft soil. As evident in Fig. 4c, the shear forces generated in the soft soil 
for the Pasternak model are greater than those of the Kerr model. Simulating the soft soil as Winkler foundation model, the shear modulus of soft soil is assumed to be zero; therefore, no shear stresses can be predicted in the soft soil as shown in Fig. 4c. When the soft soil is idealised by the Pasternak shear layer, a shear layer is attached to the bottom of the load transfer platform at the ground surface. Hence the soft soil layer underneath the LTP is exposed to shear stresses which may unrealistically exceed the shear strength of the soft soil (violating the elastic assumption used in Pasternak shear layer theory) as shown in Fig. 4c.

Fig. 5a shows the mobilised tension in the top geosynthetic layer adopting the Kerr, Winkler, and Pasternak foundation models to simulate the soft soil. The predicted maximum normalised tensions mobilised in the top geosynthetic layer simulating the soft soil adopting the Kerr and the Winkler foundation models are found to be 0.53 and $0.47 \mathrm{kN} / \mathrm{m}$ (i.e. $13 \%$ larger than corresponding value when the Kerr model is used); while in the Pasternak foundation case that value is 0.38 (i.e. $20 \%$ less than corresponding value while the Kerr model is adopted). Referring to Fig. 3a, as the LTP resting on Winkler foundation deflects greater than the Kerr foundation model, more axial strains and tensions are mobilised in the geosynthetic reinforcement than the Kerr foundation model. In contrast, the Pasternak model results in the smaller deformation of LTP when compare to the Kerr model (see Fig. 3a), hence less axial strains and tensions are mobilised in the geosynthetic reinforcement than the Kerr foundation model. Similarly, the maximum tension in the bottom geosynthetic reinforcement at the mid-span is achieved when the Winkler foundation is adopted while the minimum tension in the bottom geosynthetic reinforcement corresponding to the Pasternak foundation case, which is demonstrated in Fig. 5b. The predicted maximum normalised tension generated in the bottom geosynthetic layer, simulating the soft soil adopting the 
732 Kerr, is 0.23 , which rises to 0.27 (i.e. $15 \%$ increase) and drops to 0.15 (i.e. $44 \%$

733 decrease) while the Winkler and the Pasternak foundation models are adopted to

734 idealise the soft soil, respectively. Figs. 5a-b also display that larger tensions hence

735 larger strains are generated at the column edge than in the mid-span. Van Eekelen et al.

736 (2015) reported that strains are larger at the edges of the pile caps than in the centre of

737 the GR strips while validating the limit equilibrium models for the arching of basal

738 reinforced piled embankments. However, like a continuous reinforced beam, bottom

739 layer would be under compression at the column location (due to the assumption of

740 small cracks propagation), and since, the geosynthetics only carries tension, there

741 would be no forces mobilised in the geosynthetics. However, when geosynthetics is not

742 stiff enough and granular material is very stiff, then the tension cracks can open and go

743 through low layers of geosynthetics. In that case, the bottom geosynthetic may also

744 attract tension. To consider cracks propagating deep inside the LTP, putting both

745 geosynthetic layers under tension, Eqs. (1a) and (1b) can be used. However, for the

746 selected case study and parametric study, cracks only cross one layer of geosynthetics

747 due to the geometry and material properties used. Hence, bottom geosynthetic was not

748 subjected to tension.

749 The stress concentration ratios (SCR) when the soft soil is simulated with the Kerr,

750 the Pasternak, and the Winkler foundation models have also been examined in this

751 study. The stress concentration ratio is usually used to analyse the load distribution

752 between the columns and the soil. The higher the stress concentration ratio, the more

753 stress is transferred onto the columns. Since the stress distribution at the interface of

754 LTP and soft soil is not uniform, average stress transferred to the soil is used to

755 determine the stress concentration ratio. The stress concentration ratio can be stated as

756 (Han and Gabr, 2002; Indraratna et al., 2013a): 


$$
(S C R)_{a v g}=\frac{\sigma_{c}}{\bar{\sigma}_{s}}
$$

757 where $\sigma_{c}$ is the stress transferred to the columns and $\bar{\sigma}_{s}$ is the average stress transferred 758 to the soil on the surface. The stress concentration ratio for the soft soil idealised as the 759 Winkler foundation is larger than that of the Kerr foundation. Since the behaviour of 760 soft soil under applied load simulated with the Winkler foundation is softer than that of 761 the Kerr foundation model, almost entire applied loads transferred to the column. Very 762 less stresses transferred to the soft soil. Hence very large SCR $(\mathrm{SCR}=90)$ is observed 763 for the Winkler foundation model case. In contrast, the stress concentration ratio for the 764 soft soil idealised as the Pasternak foundation $(\mathrm{SCR}=6)$ is less than that of the Kerr 765 foundation $(\mathrm{SCR}=15)$. Inclusion of the shear layer just beneath the LTP reduces the 766 load transfer to the columns. In other words, soft soil simulated with the Pasternak 767 foundation model behaves stiffer than that of the Kerr foundation model and results in 768 the reduction of the stresses transferred to the column; hence least stress concentration 769 ratio is observed. Similar ranges of stress concentration ratios (as Kerr and Pasternak 770 foundation models) were reported by Han (2001) while stone column reinforced soft 771 soil was analysed.

772 By comparing the Kerr model to the Winkler and the Pasternak models, it is evident 773 that the combined effect of shear and compression of soft soil results in the most 774 accurate prediction of the response of LTP on soft soil. Since significant differential 775 settlement is expected near the ground surface (i.e. zone $h_{1}$ in Fig. 1a), Winkler springs 776 would be more appropriate for simulating the soil near the ground surface. However, in 777 deeper soil layers, experiencing the stress distribution and reduction in the differential 778 settlements, Pasternak shear layer attached to the springs considering both shear and 779 compressive deformations would be more appropriate. Therefore, among these, Kerr 780 foundation model is the most suitable soil foundation model to idealise the mechanistic 
behaviour of the soft soil beneath LTP. The simplified Winkler model always overpredicts the response of LTP due to the assumption of no shear resistance of soft soil. Whereas, the Pasternak model always underpredicts the deflection of LTP due to large shear resistance near the ground surface.

\subsection{Effects of column spacing}

Fig. 6a represents the effect of column spacing on the maximum settlement of LTP with one layer $(1 \times 1000 \mathrm{kN} / \mathrm{m})$ and two layers $(2 \times 1000 \mathrm{kN} / \mathrm{m})$ of geosynthetic reinforcement. It is evident from Fig. 6a that as the column spacing increases the maximum settlement of LTP which occurs at the middle of two adjacent columns also increases (as shown in Fig. 3a and as reported by Liu et al., 2015). For example, as the non-dimensional column spacing $(s / d)$ increases from 3 to 3.5 the maximum settlement is increased from $25 \mathrm{~mm}$ to $37 \mathrm{~mm}$ (i.e. $48 \%$ increase) for the granular layer with two geosynthetic layers (i.e. $2 \times 1000 \mathrm{kN} / \mathrm{m}$ ) which is shown in Fig. 6a. This is due to the accumulation of more loads on the LTP in the soft soil region for larger column spacing. Furthermore, since the area replacement ratio reduces as the spacing rises, the equivalent subgrade reaction of column decreases, and therefore the equivalent rigidity of the column supports also decreases, resulting in more settlement of LTP. Fig 6a also illustrates that the maximum settlement of the single layer geosynthetic reinforced LTP (i.e. $1 \times 2000 \mathrm{kN} / \mathrm{m}$ ) is higher than that of the double layer geosynthetic reinforced LTP (i.e. $2 \times 1000 \mathrm{kN} / \mathrm{m}$ ). For example, at $s / d=3$, the maximum settlement of LTP with single geosynthetic reinforcement (i.e. $1 \times 2000 \mathrm{kN} / \mathrm{m}$ ) is $27 \mathrm{~mm}$ which decreases to 25 $\mathrm{mm}$ while the LTP is reinforced with double geosynthetic layers (i.e. $2 \times 1000 \mathrm{kN} / \mathrm{m}$ ). As Table 3 indicates that the bending stiffness of the LTP with the single geosynthetic layer is less than that of double layer geosynthetic reinforcement. As a result, settlement is higher for single layer case. Figs. 6b shows the influence of column spacing on 
tension of geosynthetic reinforcement. It is observed that tension increases with the increase in column spacing. For example, the maximum normalised tensions in the top and the bottom geosynthetic layers increase from 0.46 to 0.57 (i.e. $24 \%$ rise) and from 0.22 to 0.28 (i.e. $27 \%$ growth), respectively, as $s / d$ increases from 3 to 3.5 . Referring to Fig. 6a, it is obvious that as the settlement of LTP increases with the increasing column spacing, the axial strain of the geosynthetic reinforcement also increases causing more tension in the geosynthetic reinforcement. Abusharar et al. (2009) also observed similar trend during an empirical analysis of a pile supported embankment. Similar ranges of strains developed in the geosynthetics were reported by Rowe and Liu (2015) while a finite element modelling of a full-scale geosynthetic-reinforced, pile-supported embankment was presented. It can be seen that the change in the tensile force with column spacing for one geosynthetic reinforcement follows the similar trend as double layers' case reported in Fig. 6b. Furthermore, for $s / d=3$, it is displayed that the one layer of geosynthetic reinforcement (i.e. $1 \times 2000 \mathrm{kN} / \mathrm{m}$ ) attracts $8 \%$ and $55 \%$ more normalised tension than the top and the bottom layer of geosynthetics, respectively in case of two layers geosynthetic reinforcement.

\subsection{Effects of LTP thickness}

As anticipated, increase in the LTP thickness results in the reduced maximum settlement of LTP which is displayed in Fig. 7a. For example, when the granular layer is reinforced with two geosynthetic layers (i.e. $2 \times 1000 \mathrm{kN} / \mathrm{m}$ ), the maximum settlement of LTP decreases $20 \%$ (i.e. from $25 \mathrm{~mm}$ to $20 \mathrm{~mm}$ ) as the non-dimensional LTP thickness $(h / d)$ increases from 1.5 to 1.75 , which is presented in Fig. 7a. Parametric study reveals that as the thickness of LTP increases the equivalent bending stiffness and shear stiffness of LTP also increase. For example, as the non-dimensional thickness of LTP $(h / d)$ increases from 1.5 to 1.75 , the equivalent bending stiffness and shear 
831 stiffness of LTP with two geosynthetic layers (i.e. $2 \times 1000 \mathrm{kN} / \mathrm{m}$ ) increase by $33 \%$ and

$83214 \%$, respectively. Thus, as the LTP becomes thicker, it becomes more inflexible which

833 results in reduced settlement as visualised in Fig 7a. Referring to Fig 7a, the maximum

834 settlement of LTP decreases when a single layer geosynthetic layer (i.e. $1 \times 2000 \mathrm{kN} / \mathrm{m}$ )

835 is replaced by two geosynthetics layers (i.e. $2 \times 1000 \mathrm{kN} / \mathrm{m}$ ). In addition, it is also

836 noticed that this reduction in the maximum settlement is more noticeable for thinner

837 LTP as compared to thicker LTP. For example, at the non-dimensional LTP thickness

$838 h / d=1.25,9 \%$ reduction in the maximum settlement of LTP is observed when a

839 single layer of geosynthetic reinforcement (i.e. $1 \times 2000 \mathrm{kN} / \mathrm{m})$ is replaced by two layers

840 of geosynthetic reinforcement (i.e. $2 \times 1000 \mathrm{kN} / \mathrm{m}$ ) as shown in Fig. $7 \mathrm{a}$. On the other

841 hand, when the non-dimensional LTP thickness $h / d=2$ is adopted, only $4 \%$ drop in

842 the maximum settlement of LTP is perceived when a single layer of geosynthetic

843 reinforcement (i.e. $1 \times 2000 \mathrm{kN} / \mathrm{m}$ ) is replaced by two layers of geosynthetic

844 reinforcement (i.e. $2 \times 1000 \mathrm{kN} / \mathrm{m}$ ). The effect of LTP thickness on the maximum

845 tension in the geosynthetic reinforcement is captured in Fig. 7b. This figure shows that

846 the maximum mobilised tension in the geosynthetic reinforcement decreases with the

847 thickness of LTP. The reason is that as LTP becomes thicker, it settles less (refer to Fig.

848 7a), and thus the axial strain of the geosynthetic reinforcement decreases, mobilising

849 less tension in the geosynthetic reinforcement. As shown in Fig. 7b, for the granular

850 layer with two geosynthetic layers $(2 \times 1000 \mathrm{kN} / \mathrm{m})$, the maximum normalised

851 mobilised tension in the top and the bottom geosynthetic layers are reduced by $13 \%$

852 and $9 \%$, respectively when $h / d$ increases from 1.5 to 1.75 . It should be noted that

853 similar trends occur for granular fill with a single geosynthetic layer (i.e. $1 \times 2000 \mathrm{kN} / \mathrm{m}$ )

854 in which the maximum mobilised tension in the geosynthetics is smaller with thicker

855 LTP compared with thinner LTP which is shown in Fig. 7b. 


\subsection{Effects of soft soil stiffness}

Effects of the soft soil stiffness on the maximum settlement of LTP are demonstrated in Fig. 8a. As evident in Fig. 8a, the maximum settlement of LTP decreases as the stiffness of soft soil increases. For example, the maximum deflection of LTP is reduced by $30 \%$ as elastic modulus of the soft soil $\left(E_{S}\right)$ increases from 1000 $\mathrm{kPa}$ to $4000 \mathrm{kPa}$ for LTP with double geosynthetics (i.e. $2 \times 1000 \mathrm{kN} / \mathrm{m}$ ). This can be explained by the fact that when soil is stiffer (i.e. soil with higher $E_{s}$ value), the spring constants $\left(k_{u}\right.$ and $\left.k_{l}\right)$ and shear modulus $(G)$ of the soil are also larger resulting in less deflection predictions for the soil. Hence, as the soil stiffness increases, the soft soil experiences less settlement, reflected in the LTP deformation. Obviously, similar relationship between the maximum deflection of LTP and the stiffness of the soft soil is observed when only one geosynthetic layer (i.e. $1 \times 2000 \mathrm{kN} / \mathrm{m}$ ) is adopted. Fig. $8 \mathrm{~b}$ shows the effect of soft soil stiffness on mobilised tension in geosynthetic reinforcement. It is observed that as the stiffness of soft soil increases tension in geosynthetic reinforcement decreases. This is due to the fact that the increase in stiffness of soft soil causes less settlement of LTP and due to this reason less axial strain and tension are induced in the geosynthetic layer. For example, as the elastic modulus of the soft soil increases from $1000 \mathrm{kPa}$ to $4000 \mathrm{kPa}$, the maximum normalised tension in the top and the bottom geosynthetic layers decreases from 0.46 to 0.3 (i.e. $35 \%$ reduction) and from 0.23 to 0.16 (i.e. $30 \%$ fall), respectively. A similar trend is observed for the case with single layer of geosynthetic as presented in Figs. 8b.

\subsection{Effects of tensile stiffness of geosynthetic reinforcement}

Fig. 9a displays the effect of tensile stiffness of geosynthetic reinforcement on the maximum settlement of LTP. As shown in Fig. 9a, the maximum settlement of LTP decreases as the tensile stiffness of geosynthetic reinforcement increases. For example, 
881 as the tensile stiffness of the each geosynthetic reinforcement for double layer case 882 increases from $1000 \mathrm{kN} / \mathrm{m}$ to $2000 \mathrm{kN} / \mathrm{m}$ (i.e. from $2 \times 1000 \mathrm{kN} / \mathrm{m}$ to $2 \times 2000 \mathrm{kN} / \mathrm{m}$ ), 883 the maximum deflection of LTP decreases $24 \%$ (i.e. from $25 \mathrm{~mm}$ to $19 \mathrm{~mm}$ ) which is 884 plotted in Fig. 9a. This can be clarified by the point that as the tensile stiffness of 885 geosynthetic reinforcement increases from $2 \times 1000 \mathrm{kN} / \mathrm{m}$ to $2 \times 2000 \mathrm{kN} / \mathrm{m}$, the 886 equivalent bending and shear stiffness of LTP becomes almost double (see Eqs. (2) and 887 (3)) which results in less deflection of LTP. Similar patterns were also observed in the 888 literature during the numerical analysis of a geosynthetic-reinforced embankments 889 over soft foundation (Rowe and Li, 2005, Han et al., 2007). Referring to Fig. 9b, due 890 to the increase in the tensile stiffness of geosynthetic reinforcement, the maximum 891 normalised tension in the geosynthetic reinforcement decreases. For example, as the 892 tensile stiffness of the each geosynthetic reinforcement increases from $1000 \mathrm{kN} / \mathrm{m}$ to $8932000 \mathrm{kN} / \mathrm{m}$ for the case of double layer, the maximum normalised tension in the top 894 layer decreases $50 \%$ (i.e. from 0.46 to 0.23 ) (see Fig. 9b). As the tensile stiffness of 895 the geosynthetic reinforcement increases, the settlement of the LTP decreases (see Fig. 896 9a), and consequently the axial strain of the geosynthetic reinforcement decreases. Liu 897 and Rowe (2015) also observed similar trend during a numerical analysis of a deep898 mixing column supported embankment. However, the tension mobilised in the 899 geosynthetic reinforcement increases. This increase in the mobilised tension is due to 900 the fact that the mobilised tension is the product of the tensile stiffness and the axial 901 strain of the geosynthetic layer (see Eqs. (39a) and (39b)). Therefore, as the tensile 902 stiffness of the geosynthetic reinforcement increases the maximum mobilised tension 903 also increases. Similar results were reported by Huang and Han (2010), and Bhasi and 904 Rajagopal (2015) for geosynthetic reinforced embankments constructed on columns 905 where numerical simulations were carried out. However, normalised tension is the ratio 
906 of mobilised tension in the geosynthetic $(T)$ and ultimate strength $\left(T_{y}\right)$ of the 907 geosynthetics. It is observed that as the tensile stiffness of the geosynthetic 908 reinforcement increases this ratio is decreased. Similar trends of the maximum 909 deflections and normalised tensions are observed for the case with single layer of 910 geosynthetic as presented in Figs. 9a-b.

911 It is mention worthy that the variations of deflection of LTP or tension in the 912 geosynthetic reinforcement with the distance between two geosynthetic layers can be 913 predicted using the proposed analytical solution in this study. It has been noticed that 914 as the distance between two layers of geosynthetic reinforcements reduces, more 915 deflection of LTP as well as the tension in geosynthetics are observed. Indeed, when 916 the geosynthetic layers are positioned closely, the effective bending stiffness of the LTP 917 (cracked LTP) is reduced contributing to more deflection of LTP and hence more 918 tension in the geosynthetics. For example, for the baseline case, when the distance 919 between two layers of geosynthetics is $2 h / 3$, the equivalent bending stiffness of LTP 920 in sagging and hogging regions is equal to $263 \mathrm{kN}$.m. However, when the distance 921 between two layers of geosynthetics is $h / 3$, the equivalent bending stiffness of LTP in 922 sagging and hogging regions is reduced to $161 \mathrm{kN} . \mathrm{m}$. Therefore, deflection of LTP as 923 well as mobilised tension in geosynthetics reinforcement increase as the spacing 924 between geosynthetic layers decreases.

925 Indeed, in this paper a simple analytical model to predict the settlement behaviour 926 of LTP on soft soil, reinforced by column inclusions such as unreinforced concrete 927 columns and reinforced piles, has been presented. To achieve the objective of the paper, 928 a closed-form solution has been developed to assess the performance of the load transfer 929 platform for a general symmetric loading pattern. Therefore, the proposed model can 930 be applied for any shape of symmetric loads from super structures such as 
931 embankments, silo, or fuel tanks where LTP over the columns is used. Indeed, since a

932 general form of symmetric external loading has been adopted in this study (see Eq.

933 (11)), user can adjust the model parameters to simulate different patterns of applied

934 loading including those obtained from existing arching theories for embankments. It

935 can be noted that a similar scenario of uniform loading was adopted by other researchers

936 (Yin, 2000a, b; Zhang et al., 2012a; Borges and Gonçalves, 2016) to investigate the

937 behaviour of load transfer platform on soft soil.Although, the loading due to arching

938 can be symmetric close to middle of the embankments, but close to the batter or slopes,

939 the loading due to arching would not be symmetric. The proposed model cannot be used

940 for asymmetric loads such as arching below batters of embankments. Thus, this is one

941 of the limitations of the proposed model.

\section{5. Conclusions}

943 The present study makes an attempt to suggest a reasonably accurate mechanical

944 model for LTP reinforced with double layers of geosynthetics on column reinforced

945 soft soil, which can be used by practicing engineers to investigate the flexural and shear

946 behaviours of the LTP. The response function of the system has been derived for

947 symmetric loading in plane strain conditions. This has been achieved by developing

948 governing differential equations for the proposed model and its solutions. In order to

949 develop analytical equations, the basic differential equations of a Timoshenko beam

950 subjected to a distributed transverse load and a foundation interface pressure, generated

951 from the Kerr foundation model were adopted. The homogeneous solution of the

952 governing sixth order nonhomogeneous differential equation was found from the roots

953 of the characteristic polynomial equation. Then adopting the method of Undetermined

954 Coefficients, the particular solution was obtained. The proposed mechanical model can 
955 be beneficial for practicing engineers in analysing the settlement response of the 956 multilayer geosynthetic reinforced granular bed overlying column improved soft soil.

957 Furthermore, soft soil idealised by the Winkler and the Pasternak foundations were 958 used to evaluate the accuracy of the adopted Kerr foundation model to detail study of 959 LTP on column improved soft soil. In general, the Winkler model produced higher 960 values of displacements, rotations, bending moments, shear forces, and tensions than 961 the reference solutions adopting the Kerr foundation model. However, the values of the 962 displacements, rotations, bending moments, shear forces, and tensions obtained from 963 Pasternak foundation model were smaller than the respective reference values adopting 964 the Kerr foundation model. Kerr foundation model predicted the response of the soft 965 soil more accurately, which were between two upper and lower bounds corresponding 966 to the Winkler and the Pasternak foundation models. Therefore, it can be concluded 967 that the Kerr foundation model is superior to the Winkler and the Pasternak models for 968 the representation of the soil response. It should be noted that this theoretical model 969 with its closed form solution may simulate the exact performance of the LTP under 970 loading. However, the presented model can be used as a tool for a better estimation of 971 the LTP behaviour with multi layers of geosynthetics, in comparison with the situation 972 that soft soil is modelled by Winkler and Pasternak foundations.

973 Furthermore, using the proposed mechanical model, response of double layer 974 geosynthetic reinforced LTP was compared with a single layer geosynthetic reinforced 975 LTP. It was observed that inclusion of the two geosynthetic layers (i.e. $2 \times 1000 \mathrm{kN} / \mathrm{m}$ ) 976 further reduced the maximum deflection of the LTP when compared to a single layer 977 (i.e. $1 \times 2000 \mathrm{kN} / \mathrm{m}$ ). However, for the double layer case, the strength of geosynthetics 978 was less utilised than that of the single layer case. It was also revealed that in the double 979 layer reinforcement, the top geosynthetic layer was more effective at the column 
980 location (in the hogging region), whereas the bottom geosynthetic layer was more 981 effective in the middle span (in the sagging region). It was also noticed that top 982 geosynthetic layer was subjected to higher mobilised tension than the bottom layer.

983 Moreover, it can be concluded that the use of one stronger geosynthetic layer (e.g. $9841 \times 2000 \mathrm{kN} / \mathrm{m}$ ) with the equivalent stiffness of two geosynthetic layers (e.g. $2 \times 1000$ $985 \mathrm{kN} / \mathrm{m}$ ), does not result in the same settlement of LTP and the tension of the geosynthetic 986 reinforcement when compared to two weaker geosynthetic layers (e.g. $2 \times 1000 \mathrm{kN} / \mathrm{m}$ ). 


\section{Acknowledgement}

988 The first author acknowledges the financial support received from Roads and 989 Maritime Services (RMS), SMEC Australia, Fulton Hogan, and Menard-Oceania. The 990 authors wish to thank A. H. M. Kamruzzaman from Roads and Maritime Services 991 (RMS) for his valuable comments at different stages of the project. 


\section{Appendix:}

993 Summary of thirteen algebraic equations obtained from the adopted boundary and continuity conditions

994 According to the boundary condition $V_{S}^{L T P}=0$, the following equation is obtained:

$$
c_{1} K_{1}-c_{2} K_{1}+c_{3} L_{1}-c_{4} M_{1}-c_{5} L_{1}-c_{6} M_{1}=R_{1}
$$

995 Boundary condition $w_{S}^{L T P^{\prime}}=0$ results:

$$
c_{1} \delta_{s}-c_{2} \delta_{s}+c_{3} \varepsilon_{s}-c_{4} \sigma_{s}-c_{5} \varepsilon_{s}-c_{6} \sigma_{s}=R_{2}
$$

996 From the boundary condition $V_{h}^{L T P}=-\left(K_{c}\right)_{e q} w_{h}^{L T P}$ following equation is obtained:

$$
\begin{aligned}
& -d_{1} e^{-\left(\frac{\delta_{h} s}{2}\right)} A_{22}+d_{2} e^{\left(\frac{\delta_{h} s}{2}\right)} B_{22}-d_{3} e^{-\left(\frac{\varepsilon_{h} s}{2}\right)}\left[C_{22} \sin \left(\frac{\sigma_{h} s}{2}\right)+E_{22} \cos \left(\frac{\sigma_{h} s}{2}\right)\right]-d_{4} e^{-\left(\frac{\varepsilon_{h} s}{2}\right)}\left[E_{22} \sin \left(\frac{\sigma_{h} s}{2}\right)-C_{22} \cos \left(\frac{\sigma_{h} s}{2}\right)\right]- \\
& d_{5} e^{\left(\frac{\varepsilon_{h} s}{2}\right)}\left[C_{22} \sin \left(\frac{\sigma_{h} s}{2}\right)-D_{22} \cos \left(\frac{\sigma_{h} s}{2}\right)\right]+d_{6} e^{\left(\frac{\varepsilon_{h} s}{2}\right)}\left[D_{22} \sin \left(\frac{\sigma_{h} s}{2}\right)+C_{22} \cos \left(\frac{\sigma_{h} s}{2}\right)\right]=R_{3}
\end{aligned}
$$

997 Assuming

$$
A_{22}=K_{2} C-\left(K_{c}\right)_{e q} ; B_{22}=K_{2} C+\left(K_{c}\right)_{e q} ; C_{22}=C M_{2} ; D_{22}=C L_{2}+\left(K_{c}\right)_{e q} ; \text { and } E_{22}=C L_{2}-\left(K_{c}\right)_{e q}
$$

998 The equation below is obtained from the boundary condition $\theta_{h}^{L T P}=0$ : 


$$
\begin{aligned}
& -d_{1} e^{-\left(\frac{\delta_{h} s}{2}\right)} A_{2}+d_{2} e^{\left(\frac{\delta_{h} s}{2}\right)} A_{2}-d_{3} e^{-\left(\frac{\varepsilon_{h} s}{2}\right)}\left[B_{2} \sin \left(\frac{\sigma_{h} s}{2}\right)-C_{2} \cos \left(\frac{\sigma_{h} s}{2}\right)\right]+d_{4} e^{-\left(\frac{\varepsilon_{h} s}{2}\right)}\left[C_{2} \sin \left(\frac{\sigma_{h} s}{2}\right)+B_{2} \cos \left(\frac{\sigma_{h} s}{2}\right)\right]- \\
& d_{5} e^{\left(\frac{\varepsilon_{h} s}{2}\right)}\left[B_{2} \sin \left(\frac{\sigma_{h} s}{2}\right)+C_{2} \cos \left(\frac{\sigma_{h} s}{2}\right)\right]-d_{6} e^{\left(\frac{\varepsilon_{h} s}{2}\right)}\left[C_{2} \sin \left(\frac{\sigma_{h} s}{2}\right)-B_{2} \cos \left(\frac{\sigma_{h} s}{2}\right)\right]=R_{4}
\end{aligned}
$$

1000 From the boundary condition $w_{S}^{L T P}=w_{h}^{L T P}$ the following equation is obtained:

$-c_{1} e^{-\delta_{s} r}-c_{2} e^{\delta_{S} r}-c_{3} e^{-\varepsilon_{S} r} \cos \sigma_{s} r-c_{4} e^{-\varepsilon_{s} r} \sin \sigma_{s} r-c_{5} e^{\varepsilon_{S} r} \cos \sigma_{s} r-c_{6} e^{\varepsilon_{s} r} \sin \sigma_{s} r+d_{1} e^{-\delta_{h} r}+d_{2} e^{\delta_{h} r}+d_{3} e^{-\varepsilon_{h} r} \cos \sigma_{h} r+$ $d_{4} e^{-\varepsilon_{h} r} \sin \sigma_{h} r+d_{5} e^{\varepsilon_{h} r} \cos \sigma_{h} r+d_{6} e^{\varepsilon_{h} r} \sin \sigma_{h} r=R_{5}$

1001 According to the boundary condition $\theta_{S}^{L T P}=\theta_{h}^{L T P}$ the following equation is obtained:

$$
\begin{aligned}
& c_{1} e^{-\delta_{s} r} A_{1}-c_{2} e^{\delta_{s} r} A_{1}+c_{3} e^{-\varepsilon_{s} r}\left(B_{1} \sin \sigma_{s} r-C_{1} \cos \sigma_{s} r\right)-c_{4} e^{-\varepsilon_{s} r}\left(C_{1} \sin \sigma_{s} r+B_{1} \cos \sigma_{s} r\right)+c_{5} e^{\varepsilon_{s} r}\left(B_{1} \sin \sigma_{s} r+C_{1} \cos \sigma_{s} r\right)+ \\
& c_{6} e^{\varepsilon_{s} r}\left(C_{1} \sin \sigma_{s} r-B_{1} \cos \sigma_{s} r\right)-d_{1} e^{-\delta_{h} r} A_{2}+d_{2} e^{\delta_{h} r} A_{2}-d_{3} e^{-\varepsilon_{h} r}\left(B_{2} \sin \sigma_{h} r-C_{2} \cos \sigma_{h} r\right)+d_{4} e^{-\varepsilon_{h} r}\left(C_{2} \sin \sigma_{h} r+\right. \\
& \left.B_{2} \cos \sigma_{h} r\right)-d_{5} e^{\varepsilon_{h} r}\left(B_{2} \sin \sigma_{h} r+C_{2} \cos \sigma_{h} r\right)-d_{6} e^{\varepsilon_{h} r}\left(C_{2} \sin \sigma_{h} r-B_{2} \cos \sigma_{h} r\right)=R_{6}
\end{aligned}
$$

1002 The following equation is after $M_{h}^{L T P}=0$ :

$$
\begin{aligned}
& d_{1} e^{-\delta_{h} r} \delta_{h} A_{2}+d_{2} e^{\delta_{h} r} \delta_{h} A_{2}+d_{3} e^{-\varepsilon_{h} r}\left(J_{2} \sin \sigma_{h} r-I_{2} \cos \sigma_{h} r\right)-d_{4} e^{-\varepsilon_{h} r}\left(I_{2} \sin \sigma_{h} r+J_{2} \cos \sigma_{h} r\right)-d_{5} e^{\varepsilon_{h} r}\left(J_{2} \sin \sigma_{h} r+\right. \\
& \left.I_{2} \cos \sigma_{h} r\right)-d_{6} e^{\varepsilon_{h} r}\left(I_{2} \sin \sigma_{h} r-J_{2} \cos \sigma_{h} r\right)=R_{7}
\end{aligned}
$$

1003 The following equation is obtained from $V_{s}^{L T P}=V_{h}^{L T P}$ : 
$-c_{1} e^{-\delta_{s} r} K_{1} C+c_{2} e^{\delta_{s} r} K_{1} C-c_{3} e^{-\varepsilon_{S} r} C\left(M_{1} \sin \sigma_{s} r+L_{1} \cos \sigma_{s} r\right)-c_{4} e^{-\varepsilon_{s} r} C\left(L_{1} \sin \sigma_{s} r-M_{1} \cos \sigma_{s} r\right)-c_{5} e^{\varepsilon_{S} r} C\left(M_{1} \sin \sigma_{s} r-\right.$

$\left.L_{1} \cos \sigma_{s} r\right)+c_{6} e^{\varepsilon_{s} r} C\left(L_{1} \sin \sigma_{s} r+M_{1} \cos \sigma_{s} r\right)+d_{1} e^{-\delta_{h} r} K_{2} C-d_{2} e^{\delta_{h} r} K_{2} C+d_{3} e^{-\varepsilon_{h} r} C\left(M_{2} \sin \sigma_{h} r+L_{2} \cos \sigma_{h} r\right)+$

$d_{4} e^{-\varepsilon_{h} r} C\left(L_{2} \sin \sigma_{h} r-M_{2} \cos \sigma_{h} r\right)+d_{5} e^{\varepsilon_{h} r} C\left(M_{2} \sin \sigma_{h} r-L_{2} \cos \sigma_{h} r\right)-d_{6} e^{\varepsilon_{h} r} C\left(L_{2} \sin \sigma_{h} r+M_{2} \cos \sigma_{h} r\right)=R_{8}$

1004 The next equation is obtained using $M_{S}^{L T P}=0$ :

$c_{1} e^{-\delta_{s} r} \delta_{s} A_{1}+c_{2} e^{\delta_{s} r} \delta_{s} A_{1}+c_{3} e^{-\varepsilon_{s} r}\left(J_{1} \sin \sigma_{s} r-I_{1} \cos \sigma_{s} r\right)-c_{4} e^{-\varepsilon_{s} r}\left(I_{1} \sin \sigma_{s} r+J_{1} \cos \sigma_{s} r\right)-c_{5} e^{\varepsilon_{s} r}\left(J_{1} \sin \sigma_{s} r+I_{1} \cos \sigma_{s} r\right)-$

$c_{6} e^{\varepsilon_{s} r}\left(I_{1} \sin \sigma_{s} r-J_{1} \cos \sigma_{s} r\right)=R_{9}$

1005 The equation below is obtained from $V_{h}^{l s}=\left(K_{c}\right)_{e q}\left(\frac{G}{C}\right) w_{h}^{l s}$ :

$$
\begin{aligned}
& d_{1} e^{-\left(\frac{\delta_{h} s}{2}\right)} L_{22}-d_{2} e^{\left(\frac{\delta_{h} S}{2}\right)} L_{22}+d_{3} e^{-\left(\frac{\varepsilon_{h} s}{2}\right)}\left[M_{22} \cos \left(\frac{\sigma_{h} s}{2}\right)-N_{22} \sin \left(\frac{\sigma_{h} s}{2}\right)\right]+d_{4} e^{-\left(\frac{\varepsilon_{h} s}{2}\right)}\left[N_{22} \cos \left(\frac{\sigma_{h} s}{2}\right)+M_{22} \sin \left(\frac{\sigma_{h} s}{2}\right)\right]- \\
& d_{5} e^{\left(\frac{\varepsilon_{h} s}{2}\right)}\left[M_{22} \cos \left(\frac{\sigma_{h} s}{2}\right)+M_{22} \sin \left(\frac{\sigma_{h} s}{2}\right)\right]+d_{6} e^{\left(\frac{\varepsilon_{h} s}{2}\right)}\left[N_{22} \cos \left(\frac{\sigma_{h} s}{2}\right)-M_{22} \sin \left(\frac{\sigma_{h} s}{2}\right)\right]=R_{10}
\end{aligned}
$$

1006 Assuming

$$
\begin{aligned}
& L_{22}=-\delta_{h}\left\{\delta_{h}{ }^{4} U_{2}-\frac{\delta_{h}{ }^{2} U_{2} k_{u}}{C}+Y_{2}\right\} ; M_{22}=-\left(\varepsilon_{h}{ }^{5}-10 \varepsilon_{h}{ }^{3} \sigma_{h}{ }^{2}+5 \varepsilon_{h} \sigma_{h}{ }^{4}\right) U_{2}+\left(\varepsilon_{h}{ }^{3}-3 \varepsilon_{h} \sigma_{h}{ }^{2}\right) \frac{U_{2} k_{u}}{C}-Y_{2} \varepsilon_{h} ; \text { and } \\
& N_{22}=\left(5 \varepsilon_{h}{ }^{4} \sigma_{h}-10 \varepsilon_{h}{ }^{2} \sigma_{h}{ }^{3}+\sigma_{h}{ }^{5}\right) U_{2}-\left(3 \varepsilon_{h}{ }^{2} \sigma_{h}-\sigma_{h}{ }^{3}\right) \frac{U_{2} k_{u}}{C}+Y_{2} \sigma_{h}
\end{aligned}
$$

1007 The following equation is obtained from $w_{s}^{l s^{\prime}}=0$ : 
$c_{1} L_{11}-c_{2} L_{11}+c_{3} M_{11}+c_{4} N_{11}-c_{5} M_{11}+c_{6} N_{11}=R_{11}$

1008

Assuming

$$
\begin{aligned}
& L_{11}=-\delta_{s}\left(\delta_{s}^{4} U_{1}-\frac{U_{1} k_{u} \delta_{s}{ }^{2}}{C}+Y_{1}\right) ; M_{11}=-\left(\varepsilon_{s}{ }^{5}-10 \varepsilon_{s}{ }^{3}{\sigma_{s}}^{2}+5 \varepsilon_{s} \sigma_{s}^{4}\right) U_{1}+\left(\varepsilon_{s}{ }^{3}-3 \varepsilon_{s} \sigma_{s}{ }^{2}\right) \frac{U_{1} k_{u}}{C}-Y_{1} \varepsilon_{s} ; \text { and } \\
& N_{11}=\left(5 \varepsilon_{s}{ }^{4} \sigma_{s}-10 \varepsilon_{s}{ }^{2} \sigma_{s}{ }^{3}+\sigma_{s}{ }^{5}\right) U_{1}-\left(3 \varepsilon_{s}{ }^{2} \sigma_{s}-\sigma_{s}{ }^{3}\right) \frac{U_{1} k_{u}}{C}+\sigma_{s} Y_{1}
\end{aligned}
$$

1009 The equation below is obtained using $w_{s}^{l s}=w_{h}^{l s}$ :

$$
c_{1} e^{-\delta_{S} r} F_{11}+c_{2} e^{\delta_{S} r} F_{11}+c_{3} e^{-\varepsilon_{S} r}\left(G_{11} \sin \sigma_{S} r+H_{11} \cos \sigma_{S} r\right)+c_{4} e^{-\varepsilon_{S} r}\left(H_{11} \sin \sigma_{S} r-G_{11} \cos \sigma_{S} r\right)-c_{5} e^{\varepsilon_{1} r}\left(G_{11} \sin \sigma_{S} r-\right.
$$

$\left.H_{11} \cos \sigma_{S} r\right)+c_{6} e^{\varepsilon_{S} r}\left(H_{11} \sin \sigma_{S} r+G_{11} \cos \sigma_{s} r\right)-d_{1} e^{-\delta_{h} r} F_{22}-d_{2} e^{\delta_{h} r} F_{22}-d_{3} e^{-\varepsilon_{h} r}\left(G_{22} \sin \sigma_{h} r+H_{22} \cos \sigma_{h} r\right)-$

$d_{4} e^{-\varepsilon_{h} r}\left(H_{22} \sin \sigma_{h} r-G_{22} \cos \sigma_{h} r\right)+d_{5} e^{\varepsilon_{h} r}\left(G_{22} \sin \sigma_{h} r-H_{22} \cos \sigma_{h} r\right)-d_{6} e^{\varepsilon_{h} r}\left(H_{22} \sin \sigma_{h} r+G_{22} \cos \sigma_{h} r\right)=R_{12}$

1010 Assuming

$$
\begin{aligned}
& G_{11}=U_{1}\left(4 \varepsilon_{s}{ }^{3} \sigma_{s}-4 \varepsilon_{s} \sigma_{s}{ }^{3}\right)-\frac{U_{1} k_{u}}{C}\left(2 \varepsilon_{s} \sigma_{s}\right) ; H_{11}=U_{1}\left(\varepsilon_{s}{ }^{4}-6 \varepsilon_{s}{ }^{2} \sigma_{s}{ }^{2}+\sigma_{s}{ }^{4}\right)-\frac{U_{1} k_{u}}{C}\left(\varepsilon_{s}{ }^{2}-\sigma_{s}{ }^{2}\right)+Y_{1} ; F_{11}=\delta_{s}{ }^{4} U_{1}-\delta_{s}{ }^{2} \frac{Q_{1} k_{u}}{C}+Y_{1} ; \\
& G_{22}=U_{2}\left(4 \varepsilon_{h}{ }^{3} \sigma_{h}-4 \varepsilon_{h} \sigma_{h}{ }^{3}\right)-\frac{U_{2} k_{u}}{C}\left(2 \varepsilon_{h} \sigma_{h}\right) ; H_{22}=U_{2}\left(\varepsilon_{h}{ }^{4}-6 \varepsilon_{h}{ }^{2} \sigma_{h}{ }^{2}+\sigma_{h}{ }^{4}\right)-\frac{U_{2} k_{u}}{C}\left(\varepsilon_{h}{ }^{2}-\sigma_{h}{ }^{2}\right)+Y_{2} ; F_{22}=\delta_{h}{ }^{4} U_{2}-\delta_{h}{ }^{2} \frac{U_{2} k_{u}}{C}+
\end{aligned}
$$

1011 The following equation is obtained from $w_{s}^{l s^{\prime}}=w_{h}^{l s^{\prime}}$ : 
$c_{1} e^{-\delta_{s} r} L_{11}-c_{2} e^{\delta_{s} r} L_{11}+c_{3} e^{-\varepsilon_{S} r}\left(M_{11} \cos \sigma_{S} r-N_{11} \sin \sigma_{S} r\right)+c_{4} e^{-\varepsilon_{S} r}\left(N_{11} \cos \sigma_{s} r+M_{11} \sin \sigma_{s} r\right)-c_{5} e^{\varepsilon_{S} r}\left(M_{11} \cos \sigma_{s} r+\right.$ $\left.N_{11} \sin \sigma_{s} r\right)+e^{\varepsilon_{s} r} c_{6}\left(N_{11} \cos \sigma_{s} r-M_{11} \sin \sigma_{s} r\right)-d_{1} e^{-\delta_{h} r} L_{22}+d_{2} e^{\delta_{h} r} L_{22}-d_{3} e^{-\varepsilon_{h} r}\left(M_{22} \cos \sigma_{h} r-N_{22} \sin \sigma_{\mathrm{h}} \mathrm{r}\right)-$ $d_{4} e^{-\varepsilon_{h} r}\left(N_{22} \cos \sigma_{h} r+M_{22} \sin \sigma_{h} r\right)+d_{5} e^{\varepsilon_{h} r}\left(M_{22} \cos \sigma_{h} r+N_{22} \sin \sigma_{h} r\right)-d_{6} e^{\varepsilon_{h} r}\left(N_{22} \cos \sigma_{h} r-M_{22} \sin \sigma_{h} r\right)=R_{13}$

1012 where

$$
\begin{aligned}
& R_{1}=0 \\
& R_{2}=0 \\
& R_{3}=-P_{0}\left(K_{c}\right)_{e q} \frac{\left(k_{u}+k_{l}\right)}{k_{u} k_{l}}-C \sum_{n=1}^{n=\infty}\left\{\left[D_{2}+E_{2}\left(\frac{2 n \pi}{s}\right)^{4}-F_{2}\left(\frac{2 n \pi}{s}\right)^{2}-1\right] p_{n h}\right\}\left(\frac{2 n \pi}{s}\right) \sin n \pi-\sum_{n=1}^{n=\infty}\left(K_{c}\right)_{e q} p_{n h} \cos n \pi \\
& R_{4}=\sum_{n=1}^{n=\infty}\left\{\left[D_{2}+E_{2}\left(\frac{2 n \pi}{s}\right)^{4}-F_{2}\left(\frac{2 n \pi}{s}\right)^{2}\right] p_{n h}+\left[\left(\frac{G F_{2} D_{s}{ }^{2}}{C^{2}}\right)\left(\frac{2 n \pi}{s}\right)^{2}+\left[\left(\frac{G F_{2} D_{s}}{C}\right)+\frac{D_{s}}{C^{2}}\right]\right] P_{n}\right\}\left(\frac{2 n \pi}{s}\right) \sin n \pi \\
& R_{5}=\sum_{n=1}^{n=\infty}\left[\left(p_{n h}-p_{n s}\right) \cos \left(\frac{2 n \pi r}{s}\right)\right] \\
& R_{6}=\sum_{n=1}^{n=\infty}\left\{\left[D_{1}+E_{1}\left(\frac{2 n \pi}{s}\right)^{4}-F_{1}\left(\frac{2 n \pi}{s}\right)^{2}\right] p_{n s}+\left[\left(\frac{G F_{1} D_{s}{ }^{2}}{C^{2}}\right)\left(\frac{2 n \pi}{s}\right)^{2}+\left[\left(\frac{G F_{1} D_{s}}{C}\right)+\frac{D_{s}}{C^{2}}\right]\right] P_{n}\right\}\left(\frac{2 n \pi}{s}\right) \sin \left(\frac{2 n \pi r}{s}\right)-\sum_{n=1}^{n=\infty}\left\{\left[D_{2}+\right.\right. \\
& \left.\left.E_{2}\left(\frac{2 n \pi}{s}\right)^{4}-F_{2}\left(\frac{2 n \pi}{s}\right)^{2}\right] p_{n h}+\left[\left(\frac{G F_{2} D_{h}{ }^{2}}{C^{2}}\right)^{2}\left(\frac{2 n \pi}{s}\right)^{2}+\left[\left(\frac{G F_{2} D_{h}}{C}\right)+\frac{D_{h}}{C^{2}}\right]\right] P_{n}\right\}\left(\frac{2 n \pi}{s}\right) \sin \left(\frac{2 n \pi r}{s}\right)
\end{aligned}
$$




$$
\begin{aligned}
& R_{7}=\sum_{n=1}^{n=\infty}\left\{\left[D_{1}+E_{1}\left(\frac{2 n \pi}{s}\right)^{4}-F_{1}\left(\frac{2 n \pi}{s}\right)^{2}\right] p_{n s}+\left[\left(\frac{G F_{1} D_{s}{ }^{2}}{C^{2}}\right)\left(\frac{2 n \pi}{s}\right)^{2}+\left[\left(\frac{G F_{1} D_{s}}{C}\right)+\frac{D_{s}}{C^{2}}\right]\right] P_{n}\right\}\left(\frac{2 n \pi}{s}\right)^{2} \cos \left(\frac{2 n \pi r}{s}\right) \\
& R_{8}=-C \sum_{n=1}^{n=\infty}\left\{\left[D_{1}+E_{1}\left(\frac{2 n \pi}{s}\right)^{4}-F_{1}\left(\frac{2 n \pi}{s}\right)^{2}-1\right] p_{n s}+\left[\left(\frac{G F_{1} D_{s}{ }^{2}}{C^{2}}\right)\left(\frac{2 n \pi}{s}\right)^{2}+\left[\left(\frac{G F_{1} D_{s}}{C}\right)+\frac{D_{s}}{C^{2}}\right]\right] P_{n}\right\}\left(\frac{2 n \pi}{s}\right) \sin \left(\frac{2 n \pi r}{s}\right)+ \\
& C \sum_{n=1}^{n=\infty}\left\{\left[D_{1}+E_{1}\left(\frac{2 n \pi}{s}\right)^{4}-F_{1}\left(\frac{2 n \pi}{s}\right)^{2}-1\right] p_{n h}+\left[\left(\frac{G F_{1} D_{h}{ }^{2}}{C^{2}}\right)\left(\frac{2 n \pi}{s}\right)^{2}+\left[\left(\frac{G F_{1} D_{h}}{C}\right)+\frac{D_{h}}{C^{2}}\right]\right] P_{n}\right\}\left(\frac{2 n \pi}{s}\right) \sin \left(\frac{2 n \pi r}{s}\right) \\
& R_{9}=\sum_{n=1}^{n=\infty}\left\{\left[D_{2}+E_{2}\left(\frac{2 n \pi}{s}\right)^{4}-F_{2}\left(\frac{2 n \pi}{s}\right)^{2}\right] p_{n h}+\left[\left(\frac{G F_{2} D_{h}{ }^{2}}{C^{2}}\right)\left(\frac{2 n \pi}{s}\right)^{2}+\left[\left(\frac{G F_{2} D_{h}}{C}\right)+\frac{D_{h}}{C^{2}}\right]\right] P_{n}\right\}\left(\frac{2 n \pi}{s}\right)^{2} \cos \left(\frac{2 n \pi r}{s}\right) \\
& R_{10}=\frac{\left(K_{c}\right)_{e q} P_{n}}{C}\left[Z_{2}\left(\frac{k_{u}+k_{l}}{k_{u} k_{l}}\right)-\left(\frac{\left(K_{c}\right)_{e q}}{C}\right)\left(\frac{U_{2}}{D_{h}}\right)\right] P_{0}-\sum_{n=1}^{n=\infty}\left\{\left[\left(\frac{U_{2}}{D_{h}}\right)+\left(\frac{U_{2}}{C}\right)\left(\frac{2 n \pi}{s}\right)^{2}\right]+\left[V_{2}\left(\frac{2 n \pi}{s}\right)^{4}+X_{2}\left(\frac{2 n \pi}{s}\right)^{2}+Z_{2}\right] p_{n h}\right\} \cos n \pi- \\
& \sum_{n=1}^{n=\infty}\left\{\left[\left(\frac{U_{2}}{D_{h}}\right)-\left(\frac{U_{2}}{C}\right)\left(\frac{2 n \pi}{s}\right)^{2}\right]\left(\frac{2 n \pi}{s}\right) P_{n}-\left[U_{2}\left(\frac{2 n \pi}{s}\right)^{4}+W_{2}\left(\frac{2 n \pi}{s}\right)^{2}+Y_{2}\right]\left(\frac{2 n \pi}{s}\right) p_{n h}\right\} \sin n \pi \\
& R_{11}=\sum_{n=1}^{n=\infty} \frac{U_{1}}{C}\left(\frac{2 n \pi}{s}\right)^{3} P_{n} \\
& R_{12}=\left[\frac{\left(k_{1}+k_{2}\right)\left(Y_{2}-Y_{1}\right)}{k_{1}}+\left(\frac{U_{1}}{D_{s}}-\frac{U_{2}}{D_{h}}\right)\right] P_{0}+\sum_{n=1}^{n=\infty}\left[\left(\frac{U_{1}}{D_{s}}-\frac{U_{2}}{D_{h}}\right)-\left(\frac{U_{1}}{C}-\frac{U_{2}}{C}\right)\left(\frac{2 n \pi}{s}\right)^{2}\right] P_{n} \cos \left(\frac{2 n \pi r}{s}\right)-\sum_{n=1}^{n=\infty}\left[\left(1+\frac{U_{1} k_{u}{ }^{2}}{C G}\right)-\left(\frac{U_{1} k_{u}}{C}\right)\left(\frac{2 n \pi}{s}\right)^{2}+\right. \\
& \left.U_{1}\left(\frac{2 n \pi}{s}\right)^{4}\right] p_{n s} \cos \left(\frac{2 n \pi r}{s}\right)+\sum_{n=1}^{n=\infty}\left[\left(1+\frac{U_{2} k_{u}{ }^{2}}{C G}\right)+\left(\frac{U_{2} k_{u}}{C}\right)\left(\frac{2 n \pi}{s}\right)^{2}+U_{2}\left(\frac{2 n \pi}{s}\right)^{4}\right] p_{n h} \cos \left(\frac{2 n \pi r}{s}\right)
\end{aligned}
$$

1013 and 


$$
\begin{aligned}
& \mathrm{R}_{13}=\sum_{n=1}^{n=\infty}\left[\left(\frac{U_{2}}{C}-\frac{U_{1}}{\mathrm{C}}\right)\left(\frac{2 n \pi}{s}\right)^{2}-\left(\frac{U_{1}}{D_{s}}+\frac{U_{2}}{D_{h}}\right)\right]\left(\frac{2 n \pi}{s}\right) P_{n} \sin \left(\frac{2 n \pi r}{s}\right)+\sum_{\mathrm{n}=1}^{\mathrm{n}=\infty}\left[U_{1}\left(\frac{2 n \pi}{s}\right)^{4}+W_{1}\left(\frac{2 n \pi}{s}\right)^{2}+Y_{1}\right]\left(\frac{2 n \pi}{s}\right) p_{n s} \sin \left(\frac{2 n \pi r}{s}\right)- \\
& \sum_{\mathrm{n}=1}^{\mathrm{n}=\infty}\left[U_{2}\left(\frac{2 n \pi}{s}\right)^{4}+W_{2}\left(\frac{2 n \pi}{s}\right)^{2}+Y_{2}\right]\left(\frac{2 n \pi}{s}\right) p_{n h} \sin \left(\frac{2 n \pi r}{s}\right)
\end{aligned}
$$

1014 Assuming

$$
W_{1}=\frac{U_{1} k_{u}}{C} ; Y_{1}=\frac{U_{1} k_{u} k_{l}}{C G}+1 ; W_{2}=\frac{U_{2} k_{u}}{C} ; \quad Y_{2}=\frac{U_{2} k_{u} k_{l}}{C G}+1 ; X_{2}=\frac{\left(K_{C}\right)_{e q} U_{2} k_{u}}{C^{2}} ; \text { and } Z_{2}=\left(\frac{\left(K_{C}\right)_{e q}}{C}\right)\left(\frac{U_{2} k_{u} k_{l}}{C G}+1\right)
$$

1015 


\section{References}

1017 Abusharar, S.W., Zheng, J.J., Chen, B.G., Yin, J.H., 2009. A simplified method for analysis of a piled embankment reinforced with geosynthetics. Geotextiles and Geomembranes. 27 (1), 39-52.

Avramidis, I.E., Morfidis, K., 2006. Bending of beams on three-parameter elastic foundation. International Journal of Solids and Structures. 43 (2), 357-375.

Bergado, D., Ruenkrairergsa, T., Taesiriy, T.Y, Balasubramaniam, A.S., 1999. Deep soil mixing used to reduce embankment settlement. Ground Improvement. 3, $145-162$.

Bhasi, A., Rajagopal, K., 2015. Geosynthetic-reinforced piled embankments: comparison of numerical and analytical methods. International Journal of Geomechanics. 15 (5), 1-12.

Borges, J.L., Gonçalves, M.S., 2016. Jet-grout column-reinforced soft soils incorporating multilayer geosynthetic-reinforced platforms. Soils and Foundations. $56(1), 57-72$.

1031 Briançon, L., Simon, B., 2012. Performance of pile-supported embankment over soft soil: Full-scale experiment. Journal of Geotechnical and Geoenvironmental Engineering. 134 (4), 551-562.

BS8006-1, 2010. Code of practice for strengthened reinforced soils and other fills. British Standards Institution.

1036 Carlsson, B., 1987. Reinforced Soil, Principles for Calculation. Terratema AB, 1037 Linköping (in Swedish).

1038 Chen, R.P., Xu, Z.Z., Chen, Y.M., Ling, D.S., Zhu, B., 2010. Field test on a piled 1039 embankment over soft ground. Journal of Geotechnical and Geoenvironmental $1040 \quad$ Engineering. $136(6), 777-785$. 
1041 Chen, Y.M., Cao, W.P., Chen, R.P., 2008. An experimental investigation of soil arching 1042 within basal reinforced and unreinforced piled embankments. Geotextiles and 1043 Geomembranes. $26(2), 164-174$.

1044 Collin, J.G., Watson, C.H., Han, J., 2005. Column-supported embankment solves time 1045 constraint for new road construction. Foundation Engineering.

1046 Cowper, G., 1966. The shear coefficient in Timoshenko's beam theory. Journal of $1047 \quad$ applied mechanics. 33 (2), 335-340.

1048 Deb, K., Basudhar, P.K., Chandra, S., 2007. Generalized model for geosynthetic1049 reinforced granular fill-soft soil with stone columns. International Journal of $1050 \quad$ Geomechanics. 7 (4), 266-276.

1051 Deb, K., 2010. A mathematical model to study the soil arching effect in stone column1052 supported embankment resting on soft foundation soil. Applied Mathematical

1054 Deb, K., Mohapatra, S.R., 2013. Analysis of stone column-supported geosynthetic1055 reinforced embankments. Applied Mathematical Modelling. 37 (5), 2943-2960.

1056 EBGEO, 2010. Empfehlungen für den Entwurf und die Berechnung von Erdkörpern 1057 mit Bewehrungen aus Geokunststoffen German Geotechnical Society 2.

1058 Farag, G.S.F., 2008. Lateral spreading in basal reinforced embankments supported by 1059 pile-like elements. In: Schiftenreihe Getechnik. Universität Kassel. Heft 20.

1060 Filonenko-Borodich, M.M., 1940. Some approximate theories of the elastic foundation.

1061 Uchenyie Zapiski Moskovskogo Gosudarstvennogo Universiteta Mekhanica 46, 1062 3-18.

1063 Filz, G.M., Smith, M.E., 2007. Net Vertical Loads on Geosynthetic Reinforcement in 1064 Column-Supported Embankments. Soil Improvement GSP 172. 1-10. 
1065 Filz, G., Sloan, J., McGuire, M.P., Collin, J., Smith, M., 2012. Column-supported embankments: Settlement and load transfer. In: Proceedings of Geo-Congress, Oakland, California.

1068

Ghosh, B., Fatahi, B., Khabbaz, H., 2016. Analytical solution to analyze LTP on column-improved soft soil considering soil nonlinearity. International Journal of Geomechanics. 04016082, 1-24.

Girout, R., Blanc, M., Thorel, L., Fagundes, D.F., Almeida, M.S.S., 2016. Arching and Deformation in a Piled Embankment: Centrifuge Tests Compared to Analytical Calculations. Journal of Geotechnical and Geoenvironmental Engineering. 142 (12).

Gray, H., 1944. Simultaneous consolidation of contiguous layers of unlike compressible soils. Proceedings of the American Society of Civil Engineers, ASCE.

Han, J., 2001. Simplified method for consolidation rate of stone column reinforced foundations. Journal of Geotechnical and Geoenvironmental Engineering. 127 (7), 597-603.

Han, J., Gabr, M.A., 2002. Numerical analysis of geosynthetic-reinforced and pilesupported earth platforms over soft soil. Journal of Geotechnical and Geoenvironmental Engineering. 128 (1), 44-53.

Han, J., Collin, J.G., Huang, J., 2004. Recent development of geosynthetic-reinforced column-supported embankments. The 55th Highway Geology Symposium. Kansas City, Missouri: 299-321.

Han, J., Oztoprak, S., Parsons, R.L., Huang, J., 2007. Numerical analysis of foundation columns to support widening of embankments. Computers and Geotechnics. 34 (6), 435-448. 
Heitz, C., 2006. Bodengewölbe unter ruhender und nichtruhender Belastung bei Berücksichtigung von Bewehrungseinlagen aus Geogittern. In: Schriftenreihe Geotechnik. Uni Kassel. Heft 19, November 2006 (in German).

Hetényi, M., 1946. Beams on elastic foundation: Theory with applications in the fields of civil and mechanical engineering, University of Michigan Press.

Hewlett, W., Randolph, M.A., 1988. Analysis of piled embankments. Ground Engineering. 21 (3), 12-18.

Horvath, J.S., 1983. Modulus of subgrade reaction: New perspective. Journal of Geotechnical Engineering. 109 (12), 1591-1596.

Huang, J., Han, J., 2009. 3D coupled mechanical and hydraulic modeling of a geosynthetic-reinforced deep mixed column-supported embankment. Geotextiles and Geomembranes. 27 (4), 272-280.

Huang, J., Han, J., Oztoprak, S., 2009. Coupled mechanical and hydraulic modeling of geosynthetic-reinforced column-supported embankments. Journal of Geotechnical and Geoenvironmental Engineering. 135 (8), 1011-1021.

Huang, J., Han, J., 2010. Two-dimensional parametric study of geosynthetic-reinforced column-supported embankments by coupled hydraulic and mechanical modeling. Computers and Geotechnics. 37 (5), 638-648.

Hutchinson, J.R., 2001. Shear coefficients for Timoshenko beam theory. Journal of Applied Mechanics. 68 (1), 87.

Indraratna, B., Rujikiatkamjorn, C., Sathananthan, I., 2005. Radial consolidation of clay using compressibility indices and varying horizontal permeability. Canadian Geotechinical Journal. 42, 1330-1341. 
1113 Indraratna, B., Basack, S., Rujikiatkamjorn, C., 2013a. Numerical solution of stone 1114 column-improved soft soil considering arching, clogging, and smear effects. 1115 Journal of Geotechnical and Geoenvironmental Engineering. 139 (3), 377-394. 1116 Indraratna, B., Ngo, N.T., Rujikiatkamjorn, C., 2013b. Deformation of coal fouled 1117 ballast stabilized with geogrid under cyclic load. Journal of Geotechnical and 1118 Geoenvironmental Engineering. $139(8), 1275-1289$.

1119 Jones, C.J.F.P., Lawson, C.R., Ayres, D.J., 1990. Geotextile reinforced piled 1120 embankments. In: Hoedt, Den (Ed.), Geotextiles, Geomembranes and Related 1121 Products, Balkema, Rotterdam.

1122 Jones, R., Xenophontos, J., 1976. On the Vlasov and Kerr foundation models. Acta $1123 \quad$ Mechanica 25, 45-49.

1124 Kempfert, H.G., Göbel, C., Alexiew, D., Heitz, C., 2004. German recommendations for 1125 reinforced embankments on pile-similar elements. In: Proceedings of EuroGeo 3, $1126 \quad$ Munich.

1127 Kerr, A.D., 1964. Elastic and viscoelastic foundation models. Journal of Applied $1128 \quad$ Mechanics. 31 (3), 491-498.

1129 Kerr, A.D., 1965. A study of a new foundation model. Acta Mechanica 1, 135-147.

1130 Khabbazian, M., Kaliakin, V.N., Meehan, C.L., 2015. Column Supported 1131 Embankments with Geosynthetic Encased Columns: Validity of the Unit Cell 1132 Concept. Geotechnical and Geological Engineering. 33 (3), 425-442.

1133 Kneifati, M.C., 1985. Analysis of plates on a Kerr foundation model. Journal of 1134 Engineering Mechanics. 111 (11), 1325-1342.

1135 Lei, G.H., Fu, C.W., Ng, C.W.W., 2016. Vertical-drain consolidation using stone 1136 columns: An analytical solution with an impeded drainage boundary under multi1137 ramp loading. Geotextiles and Geomembranes. 44 (1), 122-131. 
1138 Liu, H.L., Ng, C.W.W., Fei, K., 2007. Performance of a geogrid-reinforced and pilesupported highway embankment over soft clay: Case study. Journal of

1140 Geotechnical and Geoenvironmental Engineering. 133 (12), 1483-1493.

1141 Liu, H., Kong, G., Chu, J., Ding, X., 2015. Grouted gravel column-supported highway 1142 embankment over soft clay: case study. Canadian Geotechnical Journal. 52 (11), $1143 \quad 1725-1733$.

1144 Liu, K.W., Rowe, R.K., 2015. Numerical study of the effects of geosynthetic 1145 reinforcement viscosity on behaviour of embankments supported by deep1146 mixing-method columns. Geotextiles and Geomembranes. 43 (6), 567-578.

1147 Low, B.K., Tang, S.K., Choa, V., 1994. Arching in piled embankments. Journal of $1148 \quad$ Geotechical Engineering. 120 (11), 1917-1938.

1149 Maheshwari, P., Basudhar, P., Chandra, S., 2004. Analysis of beams on reinforced 1150 granular beds. Geosynthetics International. 11 (6), 470-480.

1151 Maheshwari, P., Viladkar, M.N., 2009. A mathematical model for beams on 1152 geosynthetic reinforced earth beds under strip loading. Applied Mathematical $1153 \quad$ Modelling. 33 (4), 1803-1814.

1154 Marston, A., Anderson, A.O., 1913. The theory of loads on pipes in ditches: and tests 1155 of cement and clay drain tile and sewer pipe, Iowa State College of Agriculture $1156 \quad$ and Mechanic Arts.

1157 MathWorks, MATLAB R2016b Optimization Toolbox ${ }^{\text {TM }}$ User's Guide. U.S.A.

1158 McGuire, M., Sloan, J., Collin, J., Filz, G., 2012. Critical height of column-supported 1159 embankments from Bench-Scale and field-scale tests. In: TC 211 International 1160 Symposium on Ground Improvement IS-GI Brussels, ISSMGE.

1161 McKelvey, J.A., 1994. The anatomy of soil arching. Geotextiles and Geomembranes. $1162 \quad 13(5), 317-329$. 
1163 Morfidis, K., 2007. Exact matrices for beams on three-parameter elastic foundation. 1164 Computers and Structures. 85 (15-16), 1243-1256.

1165 Naughton, P., 2007. The significance of critical height in the design of piled 1166 embankments. Soil Improvement, 1-10.

1167 Nogami, T., Yong, T.Y., 2003. Load-settlement analysis of geosynthetic-reinforced soil with a simplified model. Soils and Foundations. 43 (3), 33-42.

1169 Nunez, M.A., Briançon, L., Dias, D., 2013. Analyses of a pile-supported embankment over soft clay: Full-scale experiment, analytical and numerical approaches. Engineering Geology. 153, 53-67.

1172 Parsa-Pajouh, A., Fatahi, B., Khabbaz, H., 2016. Experimental and numerical investigations to evaluate two-dimensional modeling of vertical drain-assisted preloading. International Journal of Geomechanics. 16 (1).

Pasternak, P.L., 1954. On a new method of analysis of an elastic foundation by means of two constants [Gosudarstvennoe Izdatelstvo Literaturi po Stroitelstvu I

Qu, C.Z., 2009. Deformation of geocell with different tensile and compressive modulus. Journal of Geotechnical Engineering.

1180 Rogbeck, Y., Gustavsson, S., Södergren, I., Lindquist, D., 1998. Reinforced piled embankments in Sweden e design aspects. In: Proceedings of the Sixth

1183 Rowe, R.K., Li, A.L., 2005. Geosynthetic-reinforced embankments over soft 1184 foundations. Geosynthetics International. 12 (1), 50-85.

1185 Rowe, R.K., Liu, K.W., 2015. Three-dimensional finite element modelling of a full1186 scale geosynthetic-reinforced, pile-supported embankment. Canadian 1187 Geotechnical Journal. 52 (12), 2041-2054. 
1188 Russell, D., Pierpoint, N., 1997. An assessment of design methods for piled 1189 embankments. Ground Engineering, 39-44.

1190 Shukla, S.K., Yin, J.H., 2003. Time-dependent settlement analysis of a geosynthetic1191 reinforced soil. Geosynthetics International. 10 (2), 70-76.

1192 Smith, M.E., 2005. Design of bridging layers in geosynthetic-reinforced column1193 supported embankments. Doctoral Dissertation, Virginia Tech, Blacksburg.

1194 Suksiripattanapong, C., Chinkulkijniwat, A., Horpibulsuk, S., Rujikiatkamjorn, C., 1195 Tanhsutthinon, T., 2012. Numerical analysis of bearing reinforcement earth 1196 (BRE) wall. Geotextiles and Geomembranes. 32 (1), 28-37.

1197 Svanø, G., Ilstad, T., Eiksund, G., Want, A., 2000. Alternative calculation principle for 1198 design of piled embankments with base reinforcement. In: Proceedings of the 4th $1199 \quad$ GIGS Helsinki.

1200 Tan, S.A., Tjahyono, S., Oo, K., 2008. Simplified plane-strain modeling of stone1201 column reinforced ground. Journal of Geotechnical and Geoenvironmental $1202 \quad$ Engineering. 134 (2), 185-194.

1203 Terzaghi, K., 1943. Theoretical soil mechanics. John Wiley and Sons, New York. 1204 Timoshenko, S.P., 1921. On the correction for shear of the differential equation for 1205 transverse vibrations of prismatic bars. Philosophical Magazine and Journal of $1206 \quad$ Science. 41 (245), 744-746.

1207 Van Eekelen, S.J.M., Bezuijen, A., Oung, O., 2003. Arching in piled embankments; 1208 experiments and design calculations. In: Proceedings of Foundations: 1209 Innovations, Observations, Design and Practice.

1210 Van Eekelen, S.J.M., Bezuijen, A., Van Tol, A.F., 2011. Analysis and modification of 1211 the British Standard BS8006 for the design of piled embankments. Geotextiles $1212 \quad$ and Geomembranes. 29 (3), 345-359. 
1213 Van Eekelen, S.J.M., Bezuijen, A., Lodder, H.J., Van Tol, A.F., 2012a. Model

1214 experiments on piled embankments. Part I. Geotextiles and Geomembranes. 32 $1215 \quad$ (2012), 69-81.

1216 Van Eekelen, S.J.M., Bezuijen, A., Lodder, H.J., Van Tol, A.F., 2012b. Model 1217 experiments on piled embankments. Part II. Geotextiles and Geomembranes. 32 $1218 \quad$ (2012), 82-94.

1219 Van Eekelen, S.J.M., Bezuijen, A., Van Tol, A.F., 2013. An analytical model for arching in piled embankments. Geotextiles and Geomembranes. 39, 78-102.

1221 Van Eekelen, S.J.M., Bezuijen, A., Van Tol, A.F., 2015. Validation of analytical 1222 models for the design of basal reinforced piled embankments. Geotextiles and $1223 \quad$ Geomembranes. 43 (1), 56-81.

1224 Winkler, E., 1867. Die Lehre von der Elasticitaet und Festigkeit: mit besonderer 1225 Rücksicht auf ihre Anwendung in der Technik für polytechnische Schulen. 1226 Dominicus.

1227 Yapage, N.N.S., Liyanapathirana, D.S., 2014. A parametric study of geosynthetic1228 reinforced column-supported embankments. Geosynthetics International. 21 (3), $1229 \quad 213-232$.

1230 Yin, J.H., 1997a. Modelling geosynthetic-reinforced granular fills over soft soil. 1231 Geosynthetics International. 4 (2), 165-185.

1232 Yin, J.H., 1997b. A nonlinear model of geosynthetic-reinforced granular fill over soft 1233 soil. Geosynthetics International. 4 (5), 523-537.

1234 Yin, J.H., 2000a. Closed form solution of reinforced Timoshenko beam on elastic 1235 foundation. Journal of Engineering Mechanics. 126 (8), 868-874. 
1236 Yin, J.H., 2000b. Comparative modeling study of reonforced beam on elastic foundation. Journal of Geotechnical and Geoenvironmental Engineering. 126 (3), $265-271$.

1239 Yu, Y., Bathurst, R.J., 2017. Modelling of geosynthetic-reinforced column-supported 1240 embankments using 2D full-width model and modified unit cell approach. 1241 Geotextiles and Geomembranes. 45 (2), 103-120.

1242 Zaeske, D., 2001. Zur Wirkungsweise von unbewehrten und bewehrten mineralischen Tragschichten über pfahlartigen Gründungselementen. Schriftenreihe 1244 Geotechnik, Uni Kassel. Heft 10 (in German).

1245 Zhang, L., Zhao, M., Zou, X., Zhao, H., 2010. Analysis of geocell-reinforced mattress 1246 with consideration of horizontal-vertical coupling. Computers and Geotechnics. $37(6), 748-756$.

1248 Zhang, L., Zhao, M., Hu, Y., Zhao, H., Chen, B., 2012a. Semi-analytical solutions for 1249 geosynthetic-reinforced and pile-supported embankment. Computers and $1250 \quad$ Geotechnics. 44, 167-175.

1251 Zhang, L., Zhao, M., Shi, C., Zhao, H., 2012b. Nonlinear analysis of a geocell mattress 1252 on an elastic-plastic foundation. Computers and Geotechnics. 42, 204-211.

1253 Zhao, L.S., Zhou, W.H., Fatahi, B., Li, X.B., Yuen, K.V., 2016. A dual beam model for 1254 geosynthetic-reinforced granular fill on an elastic foundation. Applied 1255 Mathematical Modelling. 40 (21-22), 9254-9268. 


\section{Notation}

The following symbols are used in this paper:

$A_{c}:$ plan area of the column $\left(\mathrm{m}^{2}\right)$;

$A_{h}$ : cross section area of the granular layer in hogging region after cracking $\left(\mathrm{m}^{2}\right)$;

$A_{s}$ : cross section area of the granular layer in sagging region after cracking $\left(\mathrm{m}^{2}\right)$;

$A_{r}:$ cross section area of the geosynthetic reinforcement $\left(\mathrm{m}^{2}\right) ;$

$a_{r}$ : area replacement ratio (non-dimensional);

$C: \quad$ shear stiffness of the beam $(\mathrm{kN} / \mathrm{m})$;

$D_{h}$ : equivalent bending stiffness of the load transfer platform in hogging region $(\mathrm{kN} . \mathrm{m})$;

$D_{s}$ : equivalent bending stiffness of the load transfer platform in sagging region (kN.m);

$d: \quad$ diameter of the column (m);

$E_{c}$ : Young's modulus of the controlled modulus column material $(\mathrm{kPa})$;

$E_{g}$ : Young's modulus of the granular material in load transfer platform $(\mathrm{kPa})$;

$E_{r}:$ elastic stiffness of the geosynthetic reinforcement $(\mathrm{kPa}) ;$

$G$ : shear modulus of the soft soil $(\mathrm{kPa})$;

$H$ : depth of the soft soil (m);

$h$ : thickness of the load transfer platform before cracking $(\mathrm{m})$;

$h_{h}$ : distance of the neutral axis from the compression surface of the load transfer platform for hogging moment (m);

$h_{s}$ : distance of the neutral axis from the compression surface of the load transfer platform for sagging moment (m);

$I_{h}$ : second moment of inertia of the granular fill about neutral axis for hogging $\left(\mathrm{m}^{3}\right)$;

$I_{s}:$ second moment of inertia of the granular fill about neutral axis for sagging $\left(\mathrm{m}^{3}\right)$;

$M$ : bending moment o (kN.m); 
n: modular ratio (non-dimensional);

$\left(K_{c}\right)_{e q}$ : equivalent modulus of the subgrade reaction for column $(\mathrm{kN} / \mathrm{m})$;

$k_{c}$ : modulus of subgrade reaction for the column $\left(\mathrm{kN} / \mathrm{m}^{2} / \mathrm{m}\right)$;

$k_{l}$ : modulus of subgrade reaction for the soft soil foundation attached to the bottom of shear layer $\left(\mathrm{kN} / \mathrm{m}^{2} / \mathrm{m}\right)$;

$k_{s c}$ : shear correction coefficient of the Timoshenko beam (non-dimensional);

$k_{u}$ : modulus of subgrade reaction for the soft soil foundation attached to LTP $\left(\mathrm{kN} / \mathrm{m}^{2} / \mathrm{m}\right)$;

$p: \quad$ transverse pressure on the beam from super structure $(\mathrm{kPa})$;

$q$ : normal stress at the interface of the beam and the soft soil $(\mathrm{kPa})$;

$S: \quad$ centre to centre spacing between the two adjacent columns (m);

$\mathrm{s}$ : clear spacing between the two adjacent columns (m);

$S_{r}:$ tensile stiffness of the geosynthetic $(\mathrm{kN} / \mathrm{m})$;

$S_{r}^{b}$ : tensile stiffness of the bottom geosynthetic reinforcement $(\mathrm{kN} / \mathrm{m})$;

$S_{r}^{t}$ : tensile stiffness of the top geosynthetic reinforcement $(\mathrm{kN} / \mathrm{m})$;

$T:$ tension mobilised in the geosynthetic layer $(\mathrm{kN} / \mathrm{m})$;

$V:$ shear force $(\mathrm{kN} / \mathrm{m})$;

$w$ : transverse deflection $(\mathrm{m})$;

$y_{h}$ : distance between the neutral axis and the centroid axis of the load transfer platform in hogging region $(\mathrm{m})$;

$y_{s}:$ distance between neutral and centroid axes of the load transfer platform in sagging region $(\mathrm{m})$;

$y_{r}^{b}:$ distance of the bottom geosynthetic layer from the centroid axis of load transfer platform $(\mathrm{m})$

$y_{r}^{t}:$ distance of the top geosynthetic layer from the centroid axis of load transfer platform (m); $v_{g}$ : Poisson's ratio of the granular material (non-dimensional); 
$v_{r}$ : Poisson's ratio of the geosynthetic reinforcement (non-dimensional);

$v_{r}^{t}$ : Poisson's ratio of the top geosynthetic reinforcement (non-dimensional);

$v_{r}^{b}$ : Poisson's ratio of the bottom geosynthetic reinforcement (non-dimensional);

$\theta$ : rotation angle of the cross section (radian). 


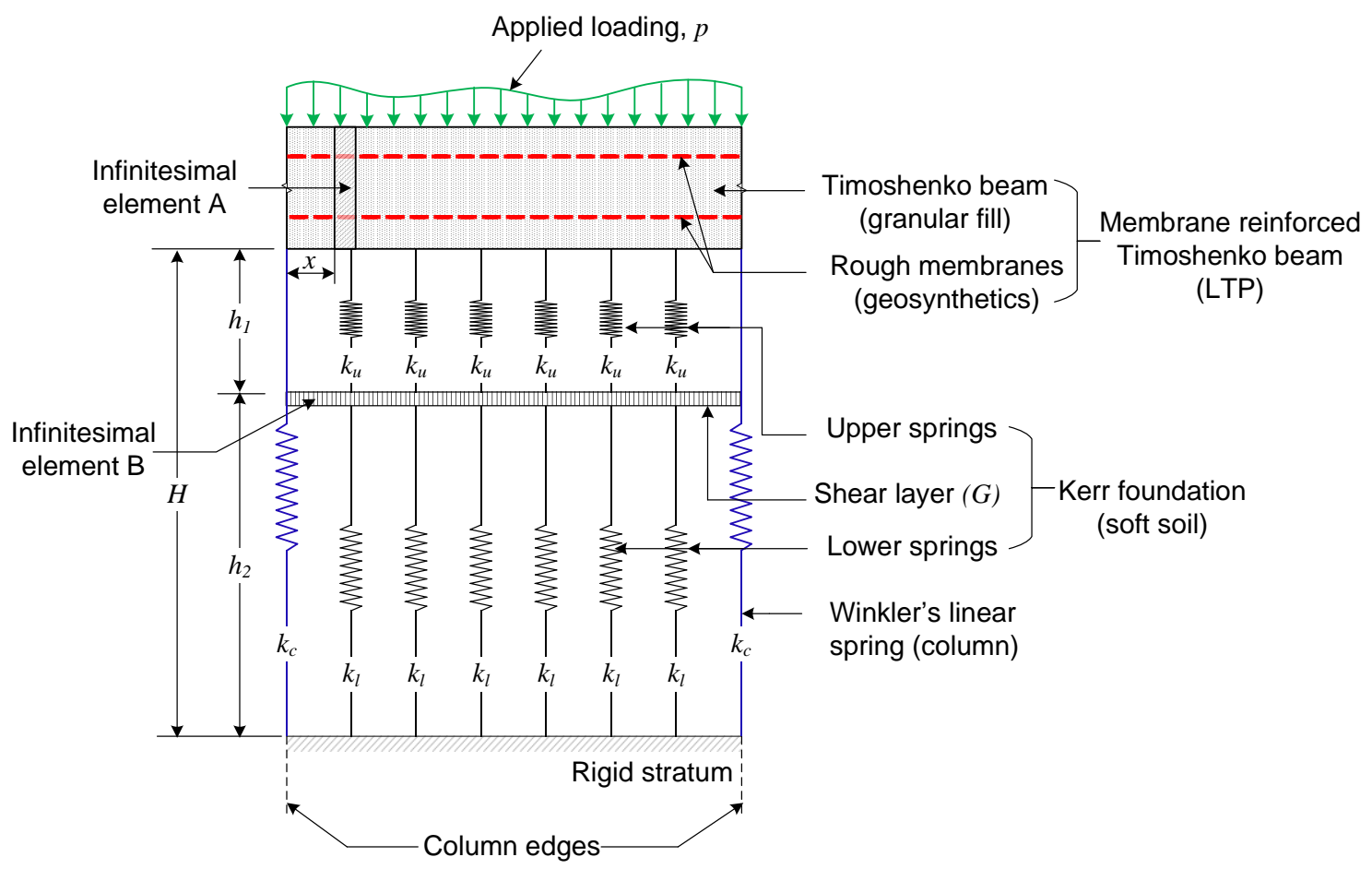

(a)

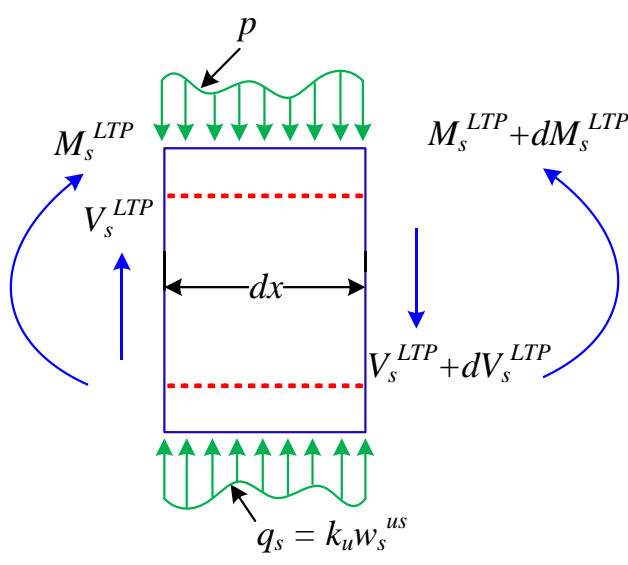

(b)

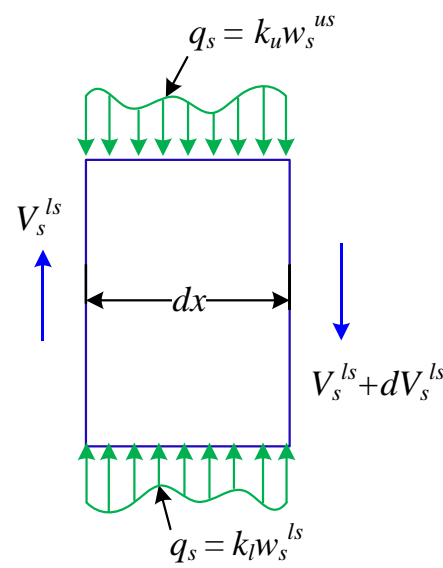

(c)

Fig. 1. Illustration of (a) proposed mechanical model of load transfer platform on column improved soft soil in plane strain condition, (b) free-body diagram of element A in sagging part, and (c) free-body diagram of element B in sagging part. 


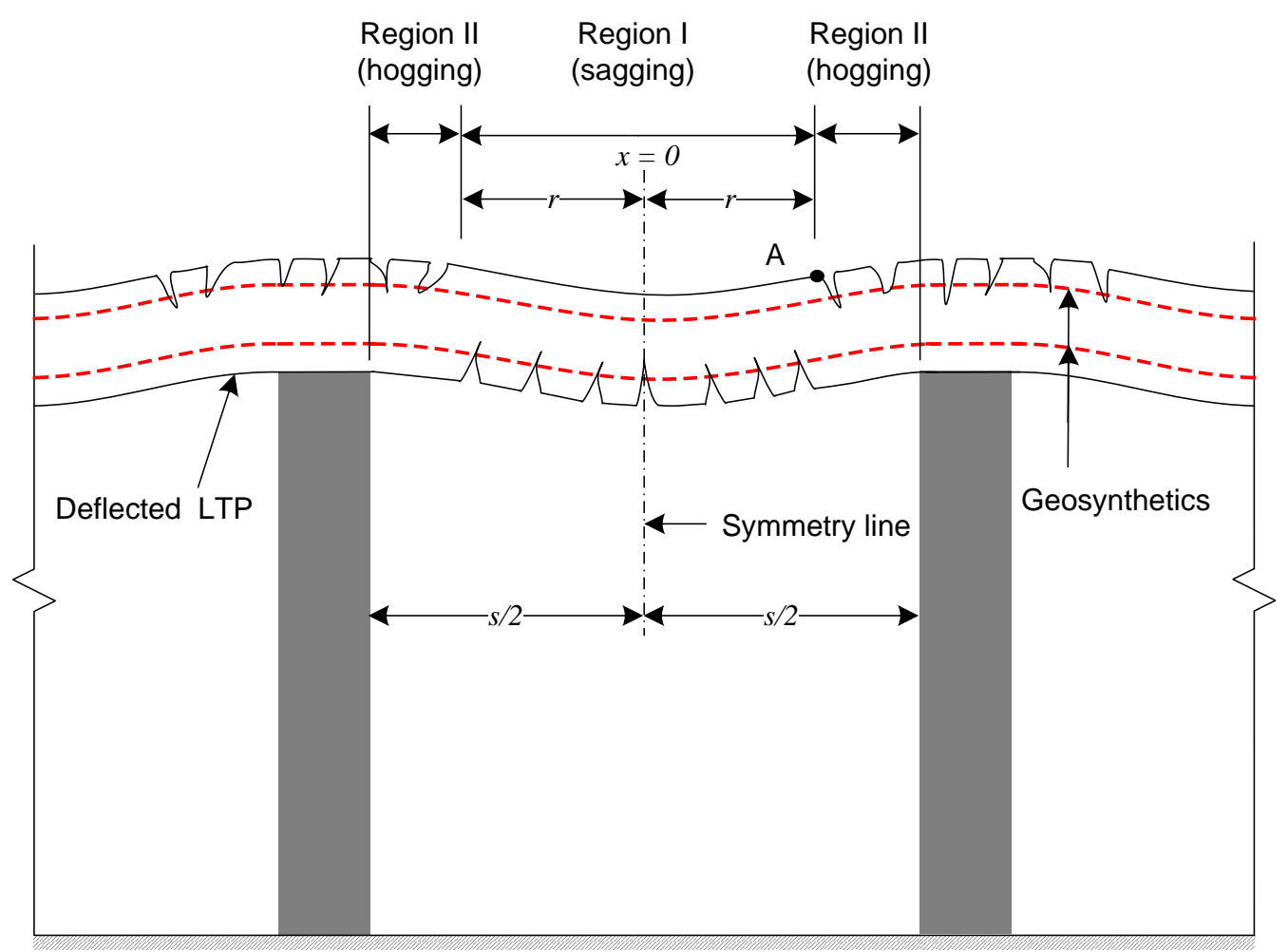

Rigid stratum

(a)

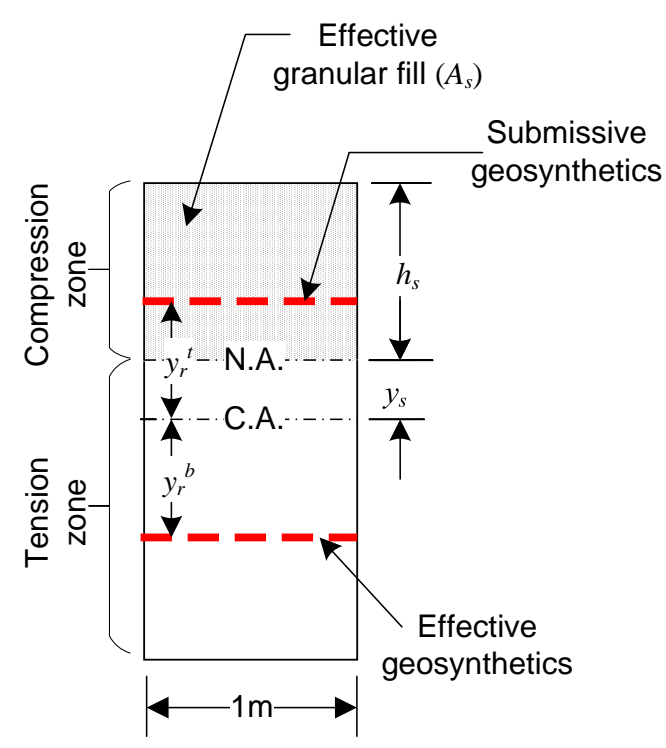

(b)

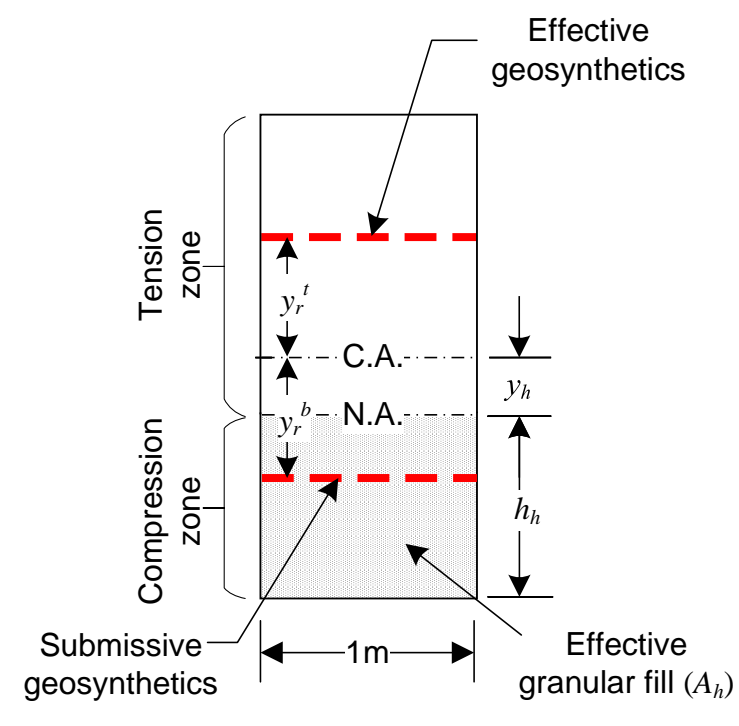

(c)

Fig. 2. Typical diagram of (a) deflection profile of load transfer platform (LTP), (b) effective cross-section of LTP in sagging region, and (c) effective cross-section of LTP in hogging region. 


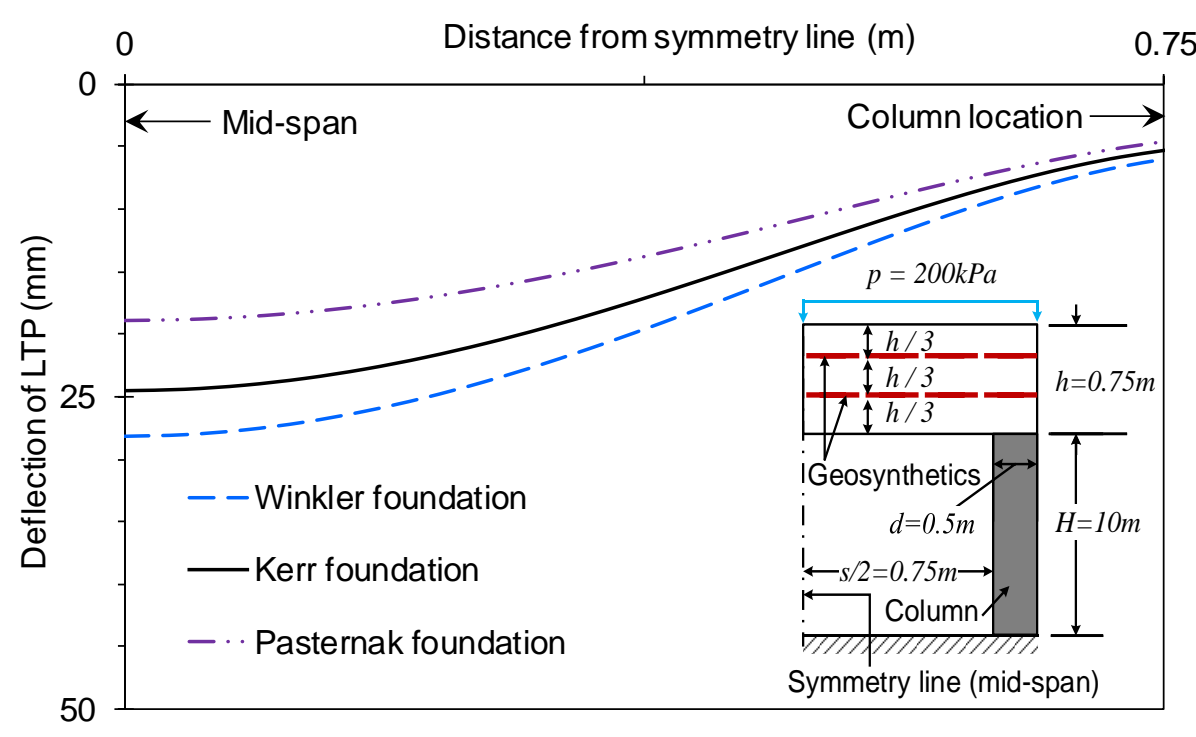

(a)

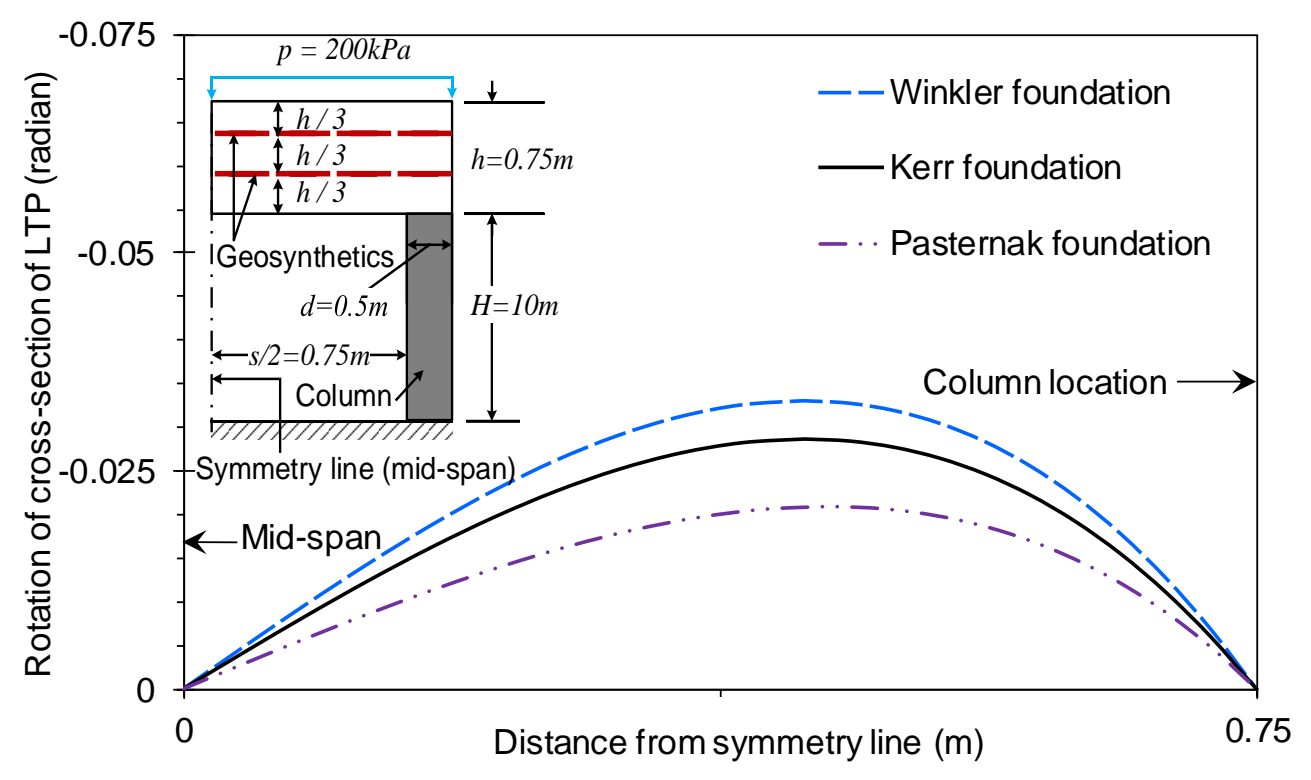

(b)

Fig. 3. Comparison of (a) settlement and (b) rotation profiles of LTP considering soft soil as Kerr, Pasternak, and Winkler foundation models. 


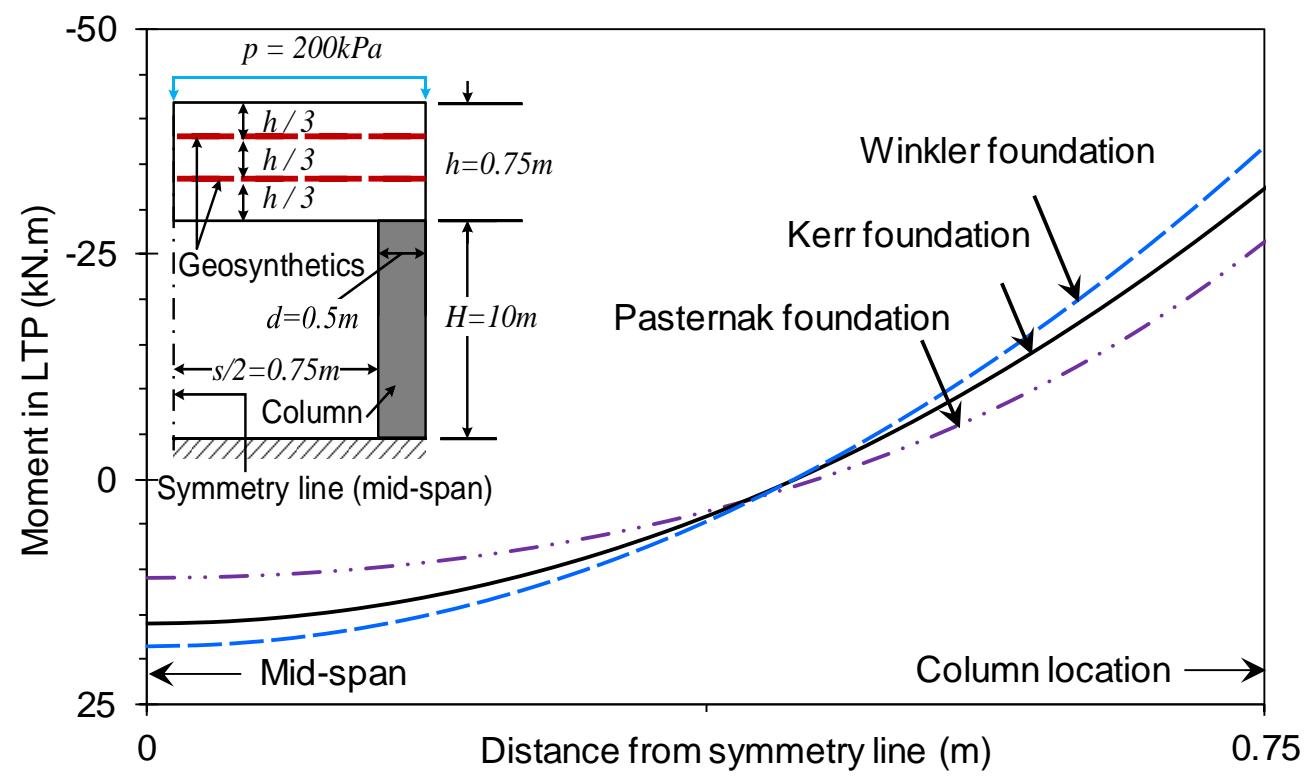

(a)

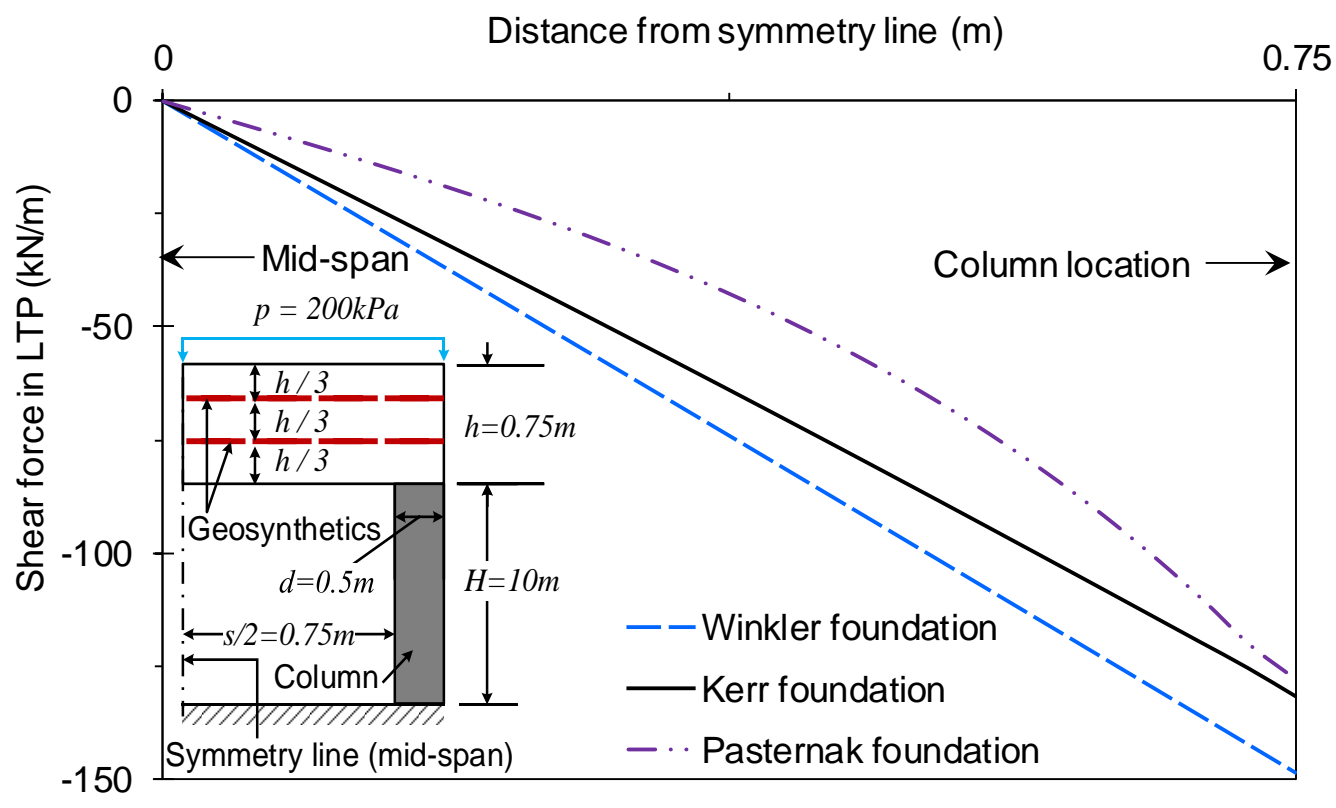

(b) 


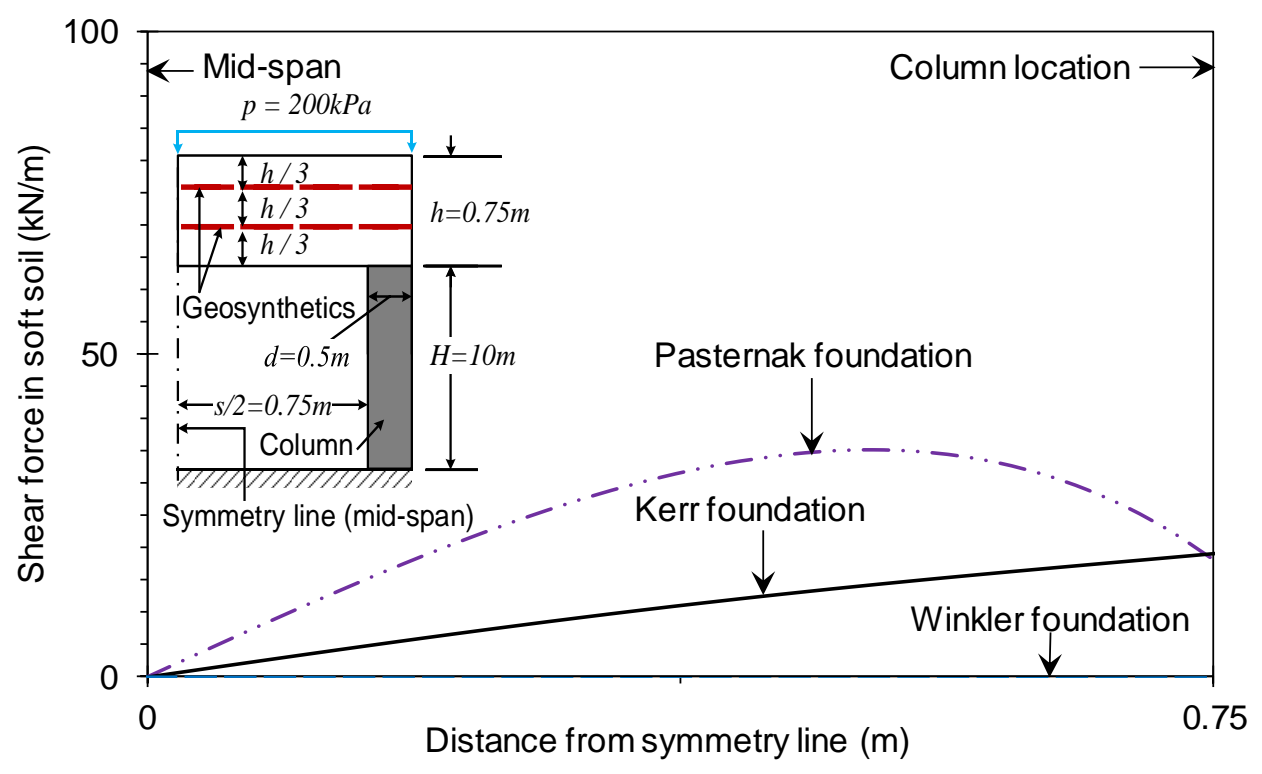

(c)

Fig. 4. Comparison of (a) bending moment of LTP, (b) shear force in LTP, and (c) shear force developed in soft soil considering soft soil as Kerr, Pasternak, and Winkler foundation models 


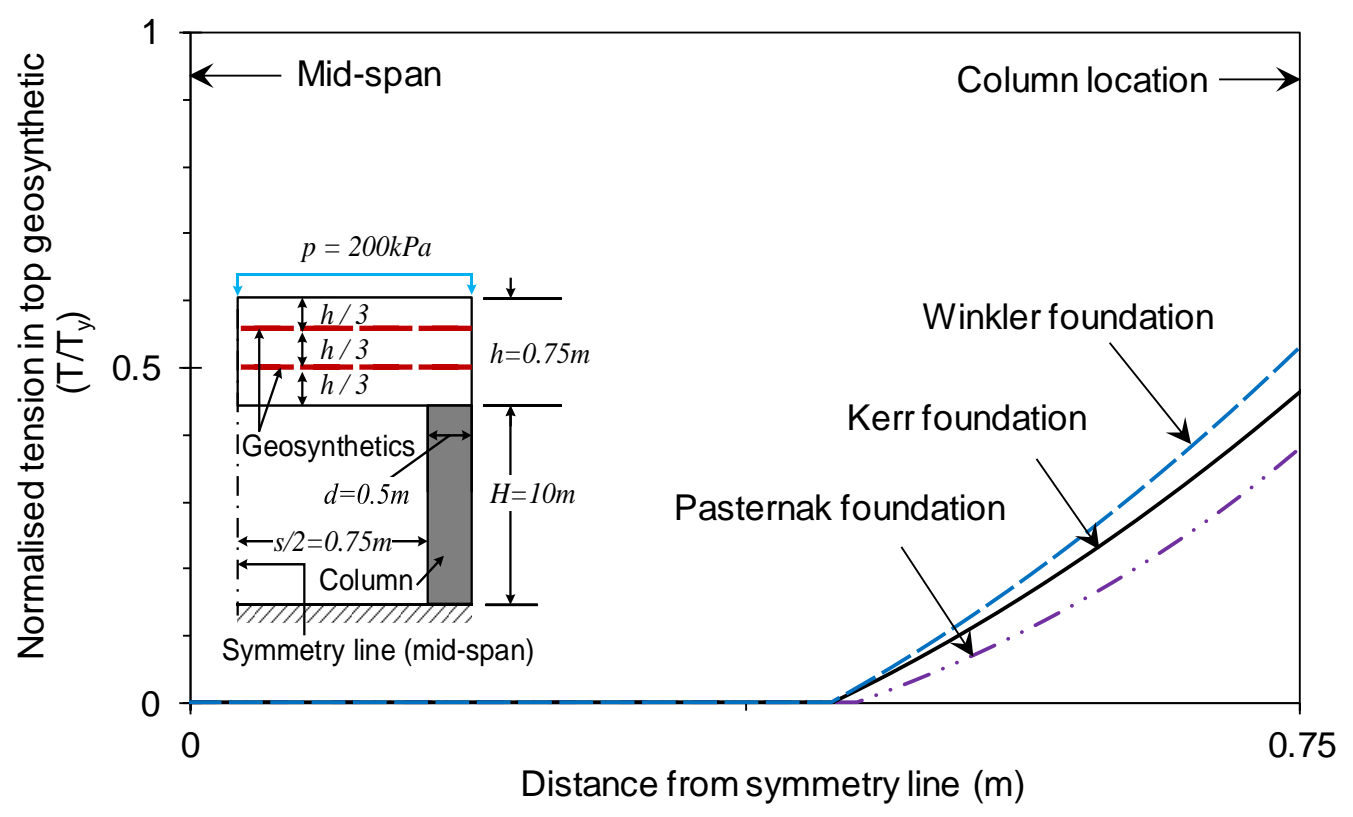

(a)

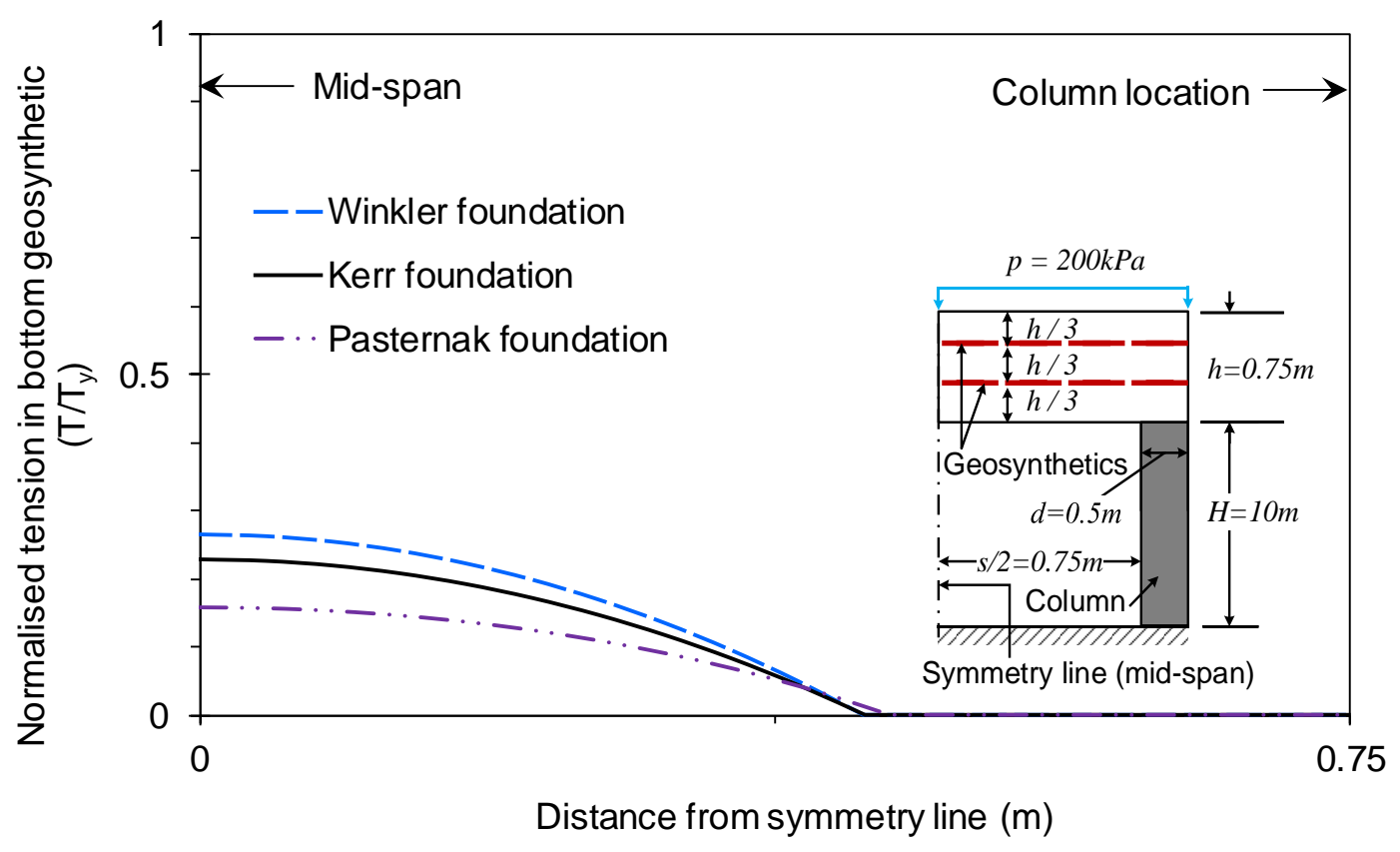

(b)

Fig. 5. Comparison of mobilised tensions in (a) top and (b) bottom geosynthetic layers considering soft soil as Kerr, Pasternak, and Winkler foundation models. 


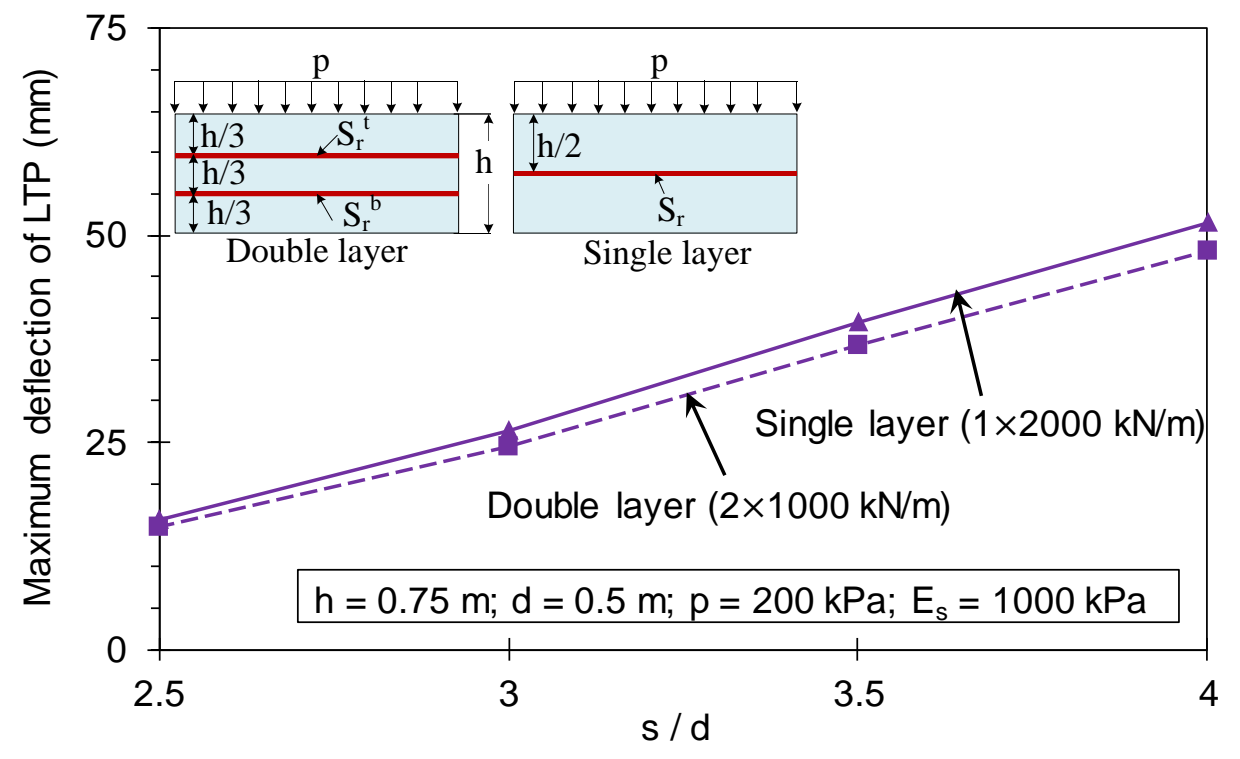

(a)

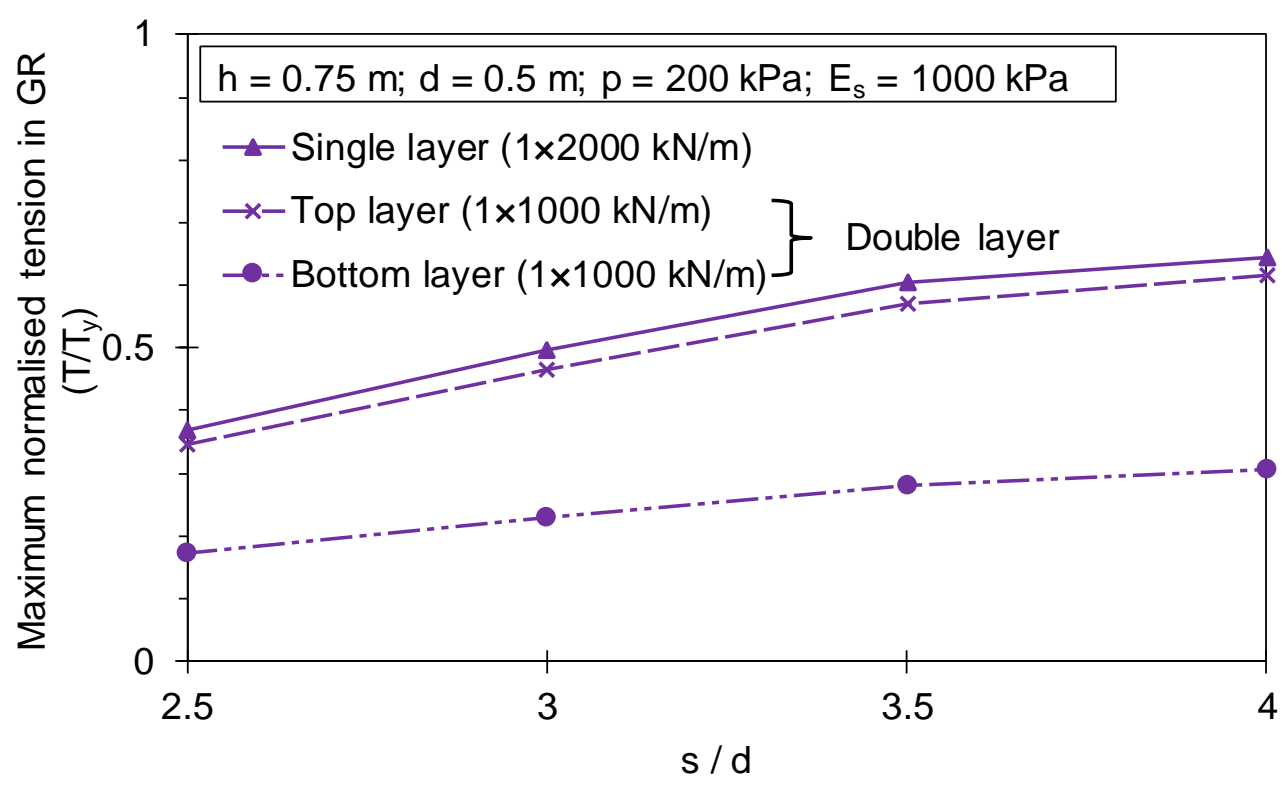

(b)

Fig. 6. Effect of column spacings for the case of LTP on Kerr foundation model on (a) the maximum deflections of LTP and (b) the maximum normalised tensions in the geosynthetics. 


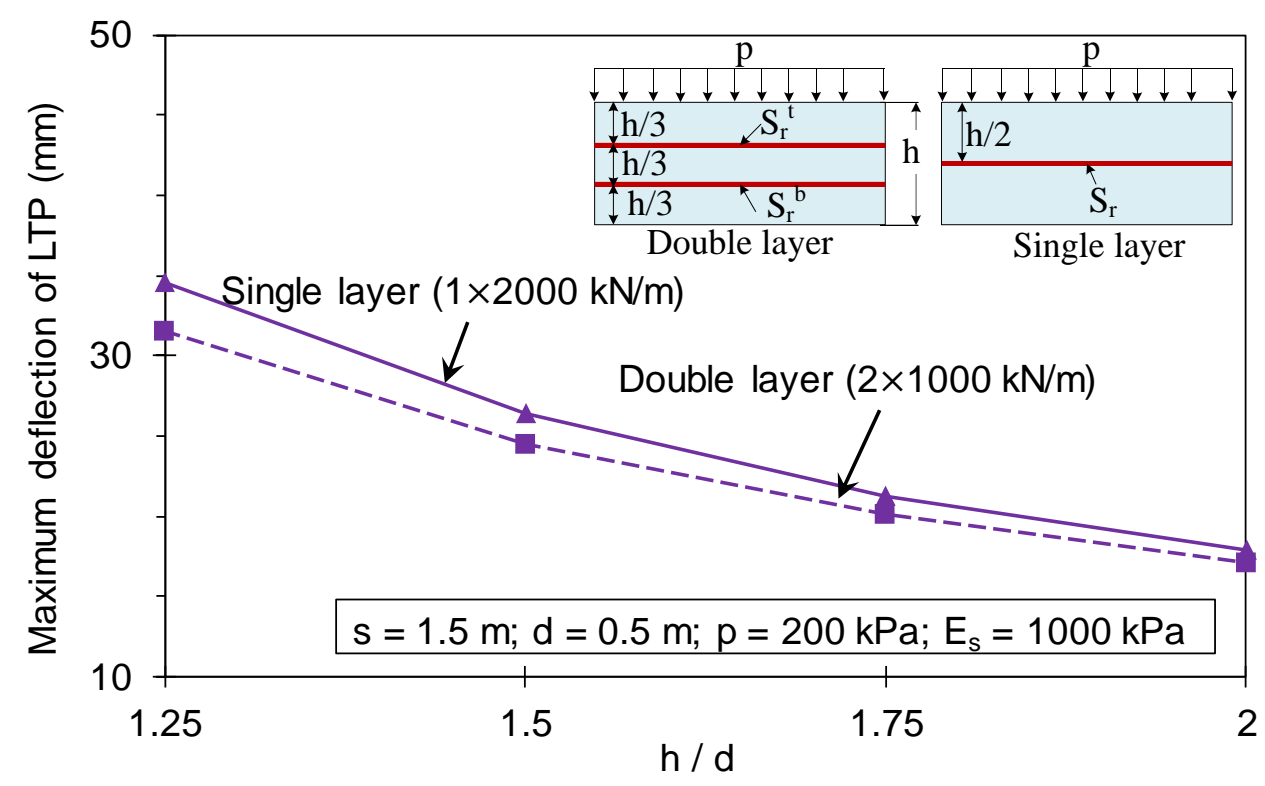

(a)

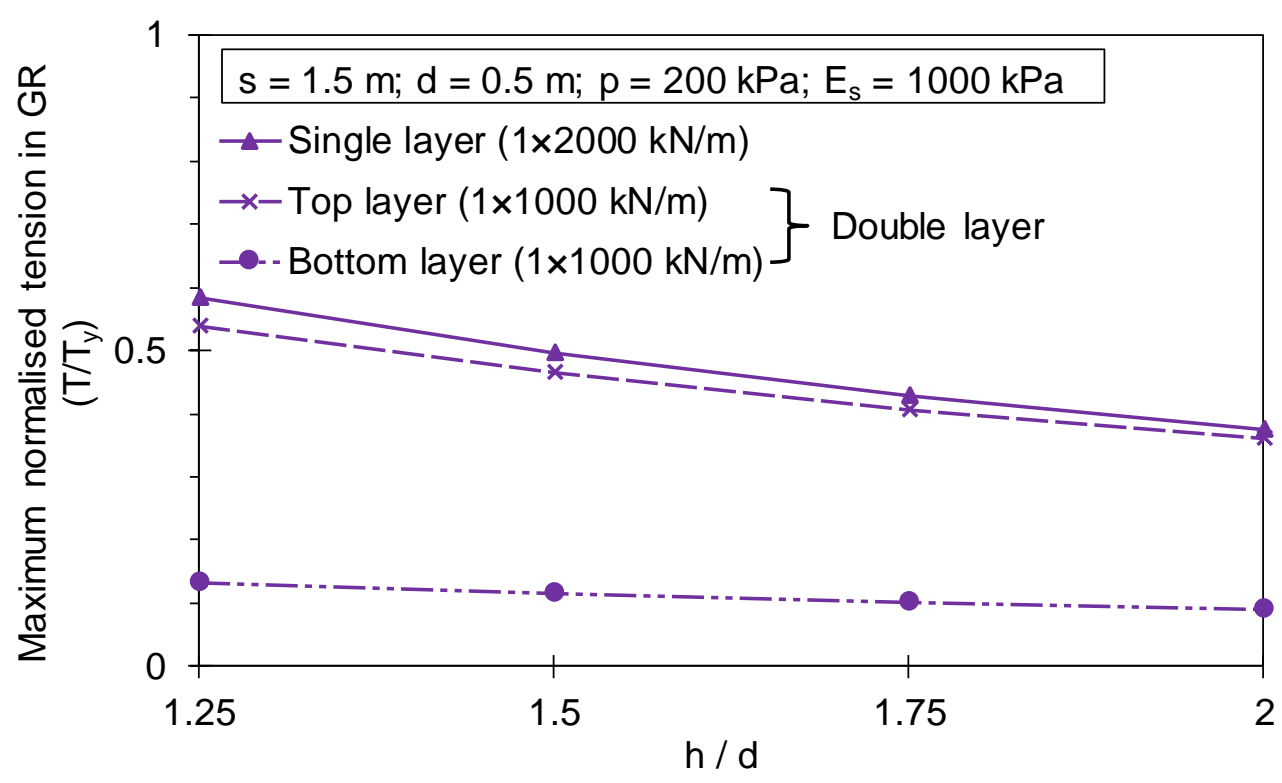

(b)

Fig. 7. Effect of LTP thicknesses for the case of LTP on Kerr foundation model on (a) the maximum deflections of LTP and (b) the maximum normalised tensions in the geosynthetics. 


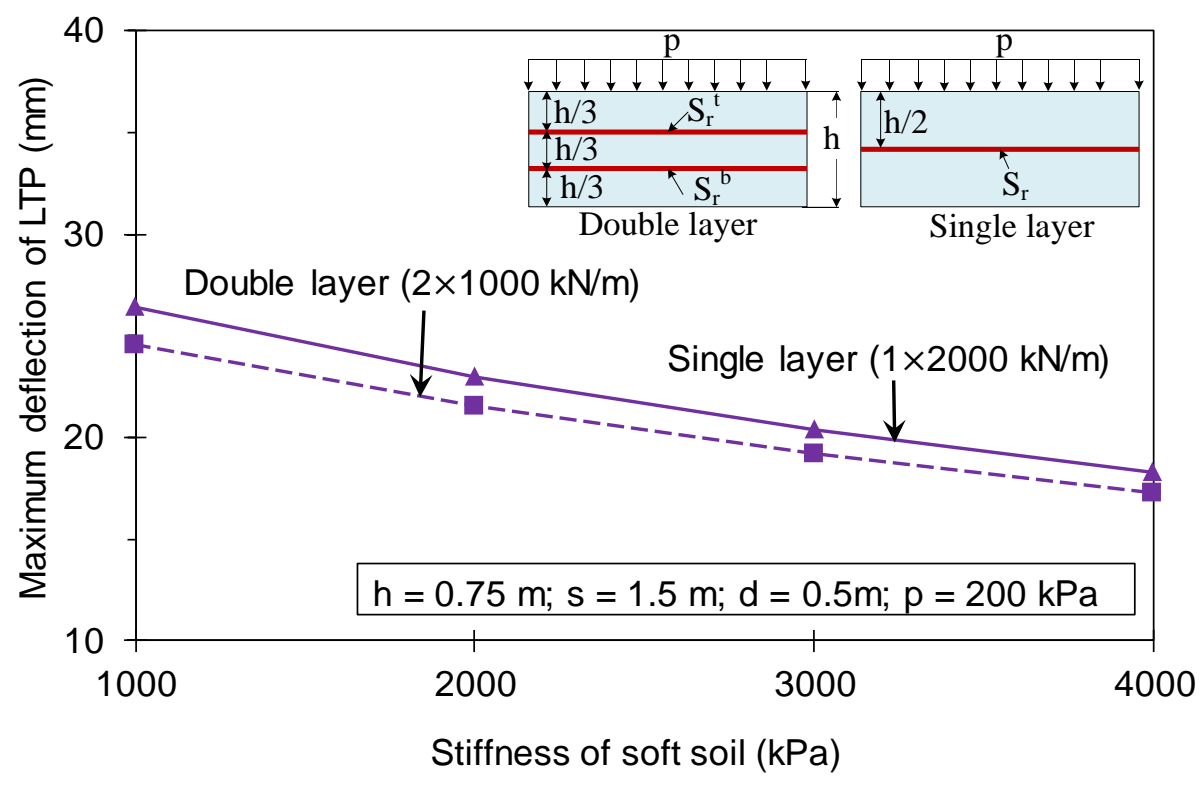

(a)

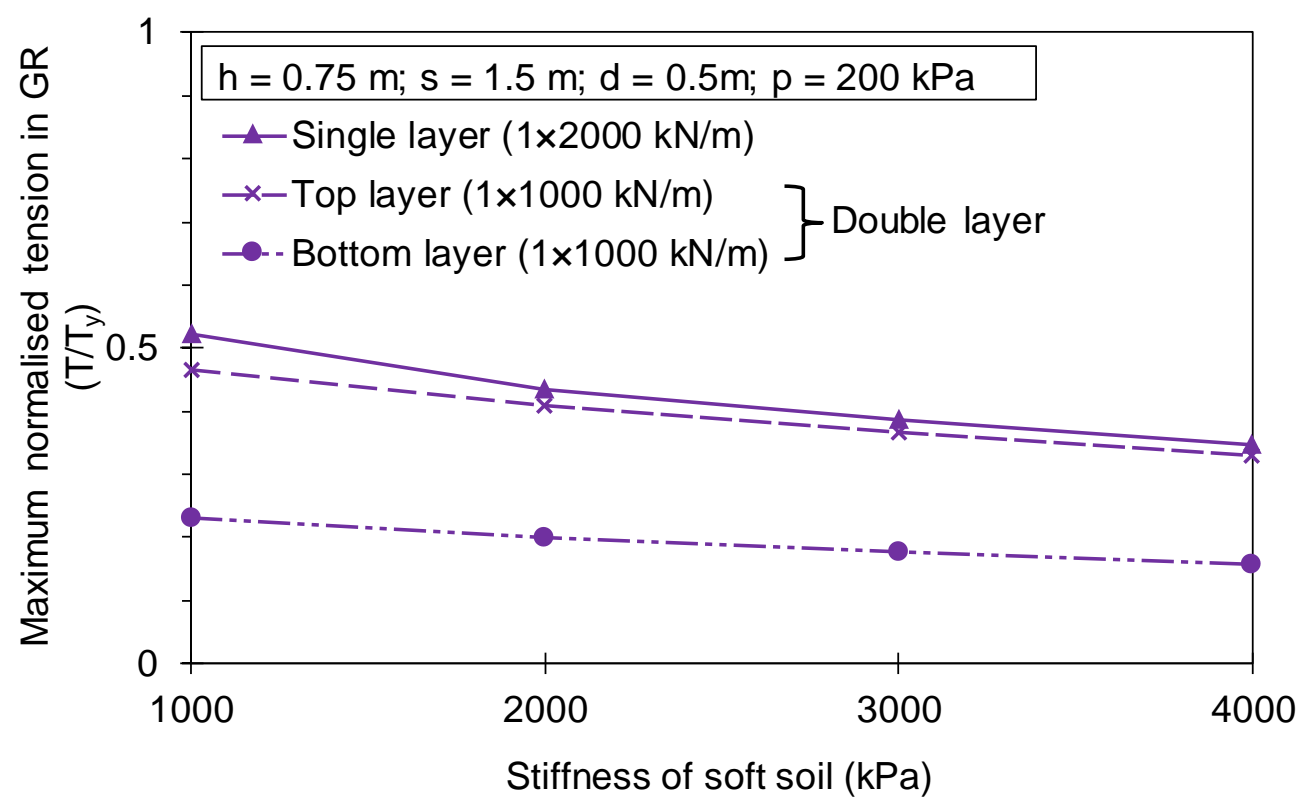

(b)

Fig. 8. Effect of soft soil stiffnesses for the case of LTP on Kerr foundation model on (a) the maximum deflections of LTP and (b) the maximum normalised tensions in the geosynthetics. 


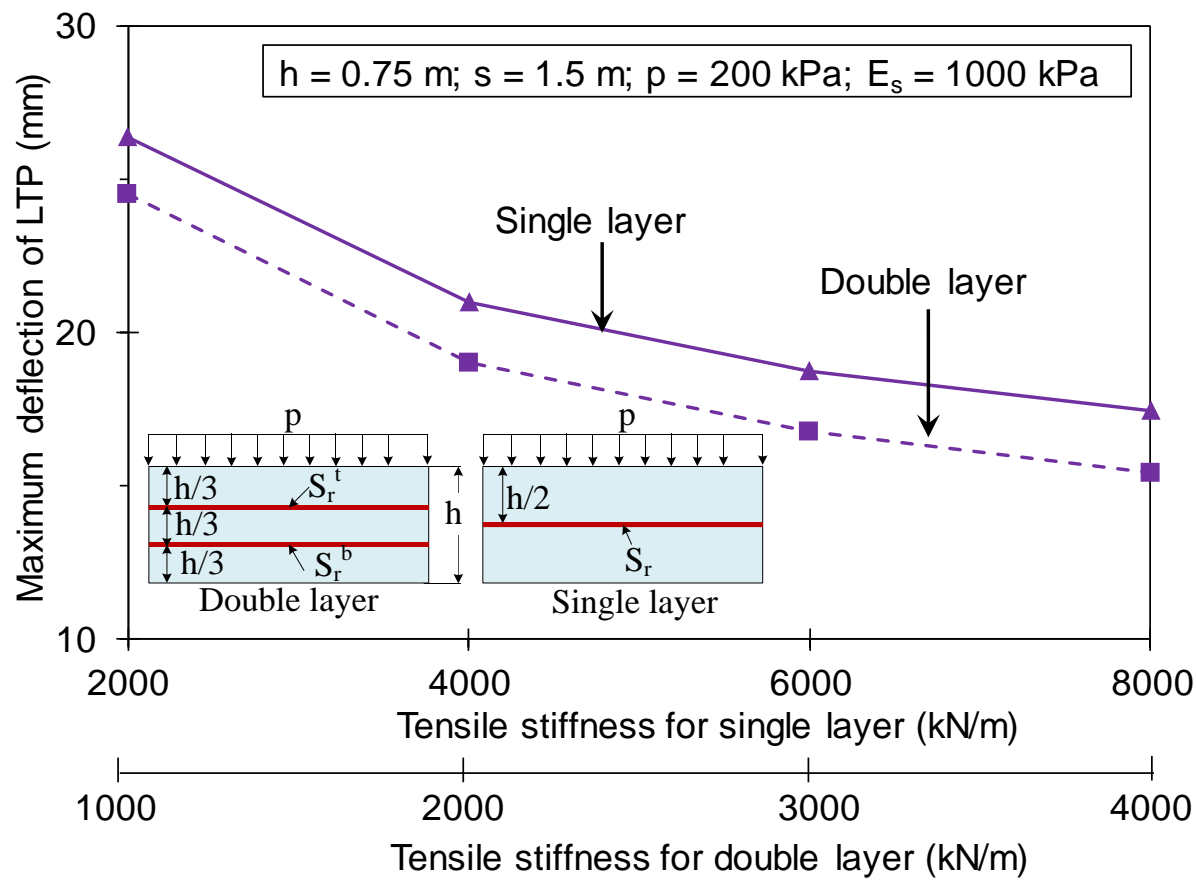

(a)

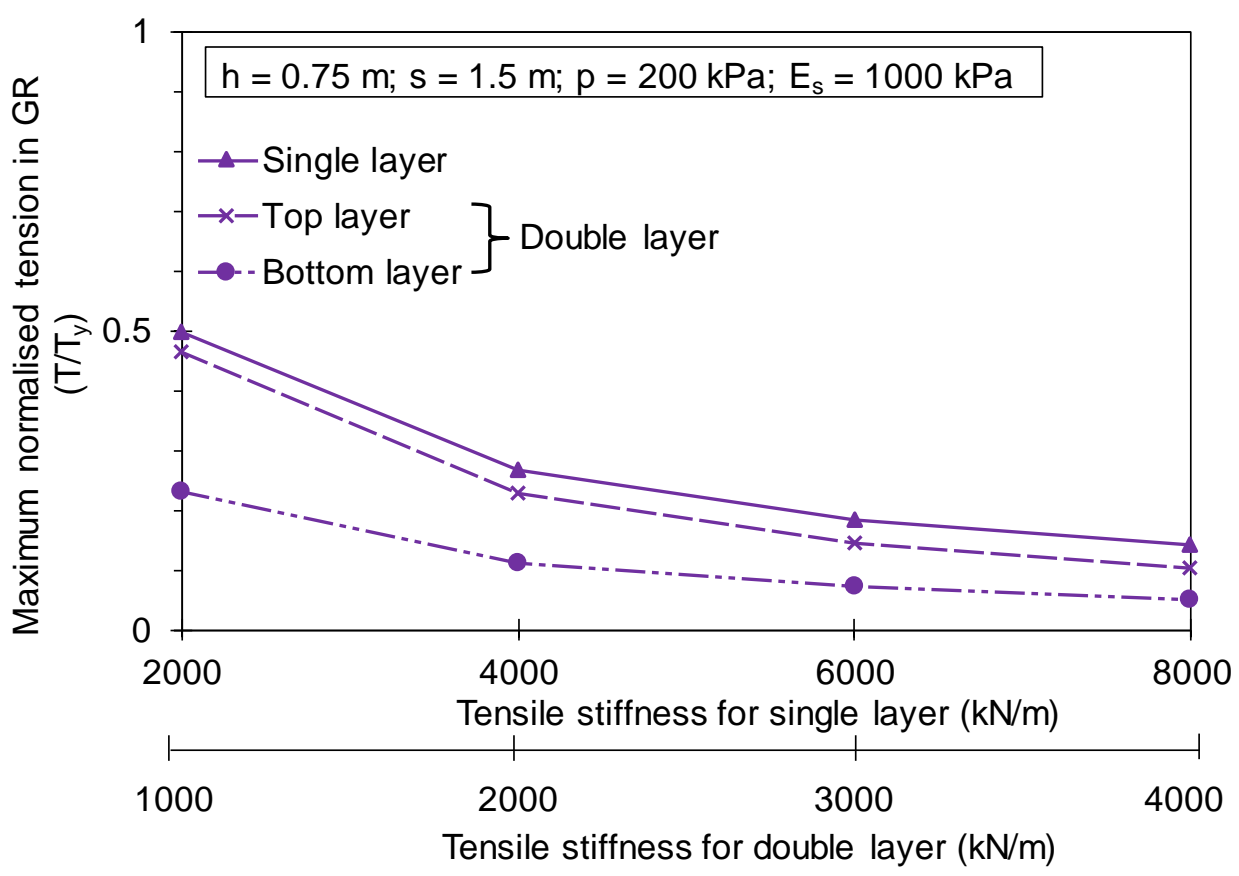

(b)

Fig. 9. Effect of tensile stiffnesses of geosynthetic reinforcement for the case of LTP on Kerr foundation model (a) the maximum deflections of LTP and (b) the maximum normalised tensions in the geosynthetics. 


\section{Table 1}

Material properties used in the baseline analysis.

\begin{tabular}{|c|c|}
\hline Material & Parameters \\
\hline Soft clay & Stiffness $\left(E_{s}\right)=1000 \mathrm{kPa}$, Poisson's ratio $\left(v_{s}\right)=0.3$ \\
\hline $\mathrm{CMC}$ & Stiffness $\left(E_{c}\right)=10,000 \mathrm{MPa}$, Poisson's ratio $\left(v_{c}\right)=0.25$ \\
\hline \multirow{2}{*}{ Geosynthetics } & $\begin{array}{ll}\text { Multilayer } & \text { Tensile stiffness }\left(S_{r}^{t}=S_{r}^{b}\right)=1000 \mathrm{kN} / \mathrm{m} \text {, } \\
& \text { Poisson's ratio }\left(v_{r}^{t}=v_{r}^{b}\right)=0.3\end{array}$ \\
\hline & $\begin{array}{l}\text { Tensile stiffness }\left(S_{r}\right)=2000 \mathrm{kN} / \mathrm{m}, \\
\text { Single layer } \\
\text { Poisson's ratio }\left(v_{r}\right)=0.3\end{array}$ \\
\hline Granular fill & Stiffness $\left(E_{g}\right)=35 \mathrm{MPa}$, Poisson's ratio $\left(v_{g}\right)=0.3$ \\
\hline
\end{tabular}




\section{Table 2}

Adopted range of parameters used in the parametric study.

\begin{tabular}{ll}
\hline Influencing factor & Range of value \\
\hline Stiffness of soft soil, $E_{s}(\mathrm{kPa})$ & $1000^{*}, 2000,3000,4000$ \\
Centre to centre spacing of columns, $S(\mathrm{~m})$ & $1.75,2.0^{*}, 2.25,2.5$ \\
& $S_{r}^{t}: 1000^{*}, 2000,3000,4000$ \\
Tensile stiffness of geosynthetics, (kN/m) & $S_{r}^{b}: 1000^{*}, 2000,3000,4000$ \\
& $S_{r}: 2000^{*}, 4000,6000,8000$ \\
Thickness of granular layer, $h(\mathrm{~m})$ & $0.625,0.75^{*}, 0.875,1$ \\
Loading, $p(\mathrm{kPa})$ & $125,150,175,200^{*}$ \\
\hline
\end{tabular}

* Parameters used for baseline analysis. 


\section{Table 3}

Calculated properties and geometries of reinforced granular layer for baseline case.

\begin{tabular}{lcc}
\hline Parameters & Double layer & Single layer \\
\hline$h_{s}(\mathrm{~m})$ & 0.14 & 0.16 \\
$h_{h}(\mathrm{~m})$ & 0.14 & 0.16 \\
$y_{s}(\mathrm{~m})$ & 0.23 & 0.22 \\
$y_{h}(\mathrm{~m})$ & 0.23 & 0.22 \\
$D_{s}(\mathrm{kN} . \mathrm{m})$ & 161 & 140 \\
$D_{h}(\mathrm{kN} . \mathrm{m})$ & 161 & 140 \\
$C(\mathrm{kN} / \mathrm{m})$ & $9.2 \times 10^{3}$ & $9.2 \times 10^{3}$ \\
\hline
\end{tabular}




\section{List of Figures}

Fig. 1. Illustration of (a) proposed mechanical model of load transfer platform on column improved soft soil in plane strain condition, (b) free-body diagram of element A in sagging part, and (c) free-body diagram of element B in sagging part.

Fig. 2. Typical diagram of (a) deflection profile of load transfer platform (LTP), (b) effective cross-section of LTP in sagging region, and (c) effective cross-section of LTP in hogging region.

Fig. 3. Comparison of (a) settlement and (b) rotation profiles of LTP considering soft soil as Kerr, Pasternak, and Winkler foundation models.

Fig. 4. Comparison of (a) bending moment of LTP, (b) shear force in LTP, and (c) shear force developed in soft soil considering soft soil as Kerr, Pasternak, and Winkler foundation models

Fig. 5. Comparison of mobilised tensions in (a) top and (b) bottom geosynthetic layers considering soft soil as Kerr, Pasternak, and Winkler foundation models.

Fig. 6. Effect of column spacings for the case of LTP on Kerr foundation model on (a) the maximum deflections of LTP and (b) the maximum normalised tensions in the geosynthetics. Fig. 7. Effect of LTP thicknesses for the case of LTP on Kerr foundation model on (a) the maximum deflections of LTP and (b) the maximum normalised tensions in the geosynthetics. Fig. 8. Effect of soft soil stiffnesses for the case of LTP on Kerr foundation model on (a) the maximum deflections of LTP and (b) the maximum normalised tensions in the geosynthetics. Fig. 9. Effect of tensile stiffnesses of geosynthetic reinforcement for the case of LTP on Kerr foundation model (a) the maximum deflections of LTP and (b) the maximum normalised tensions in the geosynthetics. 


\section{List of Tables}

Table 1 Material properties used in the baseline analysis.

Table 2 Adopted range of parameters used in the parametric study.

Table 3 Calculated properties and geometries of reinforced granular layer for base line case. 